\title{
Multi-Agent Fuzzy Reinforcement Learning for Autonomous Vehicles
}

\author{
by \\ Esther Olufunmilola Akinwumi, B.Eng
}

\begin{abstract}
A thesis submitted to the
Faculty of Graduate and Postdoctoral Affairs

in partial fulfillment of the requirements for the degree of
\end{abstract}

Master of Applied Science in Electrical and Computer Engineering

Ottawa-Carleton Institute for Electrical and Computer Engineering

Department of Systems and Computer Engineering

Carleton University

Ottawa, Ontario

January, 2020

(C) Copyright

Esther Olufunmilola Akinwumi, 2020 
The undersigned hereby recommends to the

Faculty of Graduate and Postdoctoral Affairs acceptance of the thesis

\title{
Multi-Agent Fuzzy Reinforcement Learning for Autonomous Vehicles
}

\author{
submitted by Esther Olufunmilola Akinwumi, B.Eng \\ in partial fulfillment of the requirements for the degree of
}

Master of Applied Science in Electrical and Computer Engineering

Professor Howard Schwartz, Thesis Supervisor

Professor Yvan Labiche, Chair,

Department of Systems and Computer Engineering

Ottawa-Carleton Institute for Electrical and Computer Engineering

Department of Systems and Computer Engineering

Carleton University

January, 2020 


\section{Abstract}

This thesis investigates how the evader in a pursuit-evasion differential game can learn its control strategies using the fuzzy actor-critic learning algorithm. The evader learns its control strategies while being chased by a pursuer that is also learning its control strategy in two pursuit-evasion games; the homicidal chauffeur game and the game of two cars. The simulation results presented in this thesis prove that the evader is able to learn its control strategy effectively using only triangular membership functions and only updating its output parameters. When compared with the simulation results from [1], the approach in this thesis saves a significant amount of computation time.

This thesis also introduces fuzzy-actor critic learning to an inertial missile guidance problem. The missile solely relies on its own control surfaces to intercept the target. Proportional navigation is one of the techniques in literature that can successfully guide the missile to the target. The simulation results presented in this thesis suggests that fuzzy actor-critic learning can successfully guide a missile with simple dynamics to the target. 


\section{Acknowledgments}

I thank God for the successful completion of this thesis. I thank my parents and siblings for all their love and support. My sincere gratitude to my thesis supervisor, Prof. Howard Schwartz for his patience, encouragement, guidance and funding throughout my graduate program. I would also like to thank my colleague Tolulope Akinbulire for the brainstorming sessions. Finally, a big thanks to the professors and staff of the systems and computer engineering department. 


\section{Table of Contents}

Abstract $\quad$ iii

Acknowledgments $\quad$ iv

Table of Contents $\quad$ v

List of Tables viii

List of Figures $\quad x$

Nomenclature $\quad$ xiii

1 Introduction 1

1.1 Overview . . . . . . . . . . . . . . . . . . . 1

1.2 Literature Review . . . . . . . . . . . . . . . . . . 2

1.3 Contributions and Thesis Organization . . . . . . . . . . . 5

2 Fuzzy Control Systems $\quad 8$

2.1 Fuzzy Logic Control . . . . . . . . . . . . . . . . . . 8

2.2 Fuzzy Sets and Operations . . . . . . . . . . . . . . . 9

2.2.1 Fuzzy Inference Process . . . . . . . . . . . . . . . . . . . . 11

2.2.2 Defuzzification ........................... 13

2.2.3 Function Approximation using Fuzzy Logic . . . . . . . . . . . 14 
2.2.3.1 Example 1. . . . . . . . . . . . . . 14

3 Reinforcement Learning $\quad 21$

3.1 Introduction . . . . . . . . . . . . . . . . . . 21

3.1.1 Elements of reinforcement learning . . . . . . . . . . 22

3.2 Markov Decision Processes . . . . . . . . . . . . . . . . . . 24

3.2 .1 Dynamic Programming . . . . . . . . . . . . . . . 26

3.3 Temporal-Difference Learning . . . . . . . . . . . . . . . 27

3.3.1 Q-Learning . . . . . . . . . . . . . . . . . . . . . . . . 29

3.3.2 Actor-Critic Learning . . . . . . . . . . . . . . . . . . . . . 29

3.4 Continuous State and Action Spaces _. . . . . . . . . . 30

3.4.1 Fuzzy Actor-Critic Learning . . . . . . . . . . . . . . . 31

3.4.2 $\mathrm{Q}(\lambda)$-Learning Fuzzy Inference System . . . . . . . . . . 34

3.5 Discussion . . . . . . . . . . . . . . . . . . . . . . . 38

4 Differential Games $\quad 40$

4.1 Pursuit-evasion Differential Game . . . . . . . . . . . . . 40

4.1.1 The Homicidal Chauffeur Problem $[1,2] \ldots \ldots$. . . . . . . . 41

4.1.2 The Game of Two Cars $[1,2] \ldots \ldots \ldots \ldots$

4.2 Homing Missile Guidance . . . . . . . . . . . . . . . . . 47

4.3 Summary . . . . . . . . . . . . . . . . . . . 52

5 Fuzzy Actor-Critic Learning in Pursuit Evasion Games 53

5.1 Introduction . . . . . . . . . . . . . . . 53

5.2 Simulation Results . . . . . . . . . . . . . . . . . . 54

5.2 .1 Homicidal Chauffeur Game . . . . . . . . . . . . . 56

5.2.2 Homicidal chauffuer game using QLFIS algorithm in [1] . . . 59 
5.2.3 Homicidal Chauffuer Game using the FACL Algorithm . . . . 61

5.2.4 Game of Two Cars . . . . . . . . . . . . . . . . . 77

5.2.5 Game of Two Cars using the QLFIS Algorithm from [1] . . . . 77

5.2.6 Game of Two Cars using the FACL Algorithm . . . . . . . . . 79

5.2.6.1 Homing Missile Guidance . . . . . . . . . . . 88

5.2 .7 Discussion . . . . . . . . . . . . . . . . . . 90

6 Conclusion $\quad 91$

$\begin{array}{ll}\text { List of References } & 93\end{array}$ 


\section{List of Tables}

5.1 Summary of the average number of times the rule with premise $C$ (i.e $D=R_{p}$ ) fire during 1000 episodes of the homicidal chauffeur game case 166

5.2 Summary of the average number of times the rule with premise $F$ (i.e $D=R_{p}$ ) fired during 1000 episodes of the homicidal chauffeur game

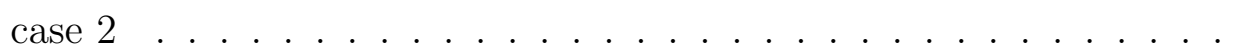

5.3 Summary of the average number of times the rule with premise $V V V C$ (i.e $D=R_{p}$ ) fired during 1000 episodes of the homicidal chauffeur game case $3 \ldots \ldots \ldots \ldots \ldots \ldots \ldots$

5.4 Summary of the number of episodes taken for the evader to learn its control strategy and percentage number of times the evader escapes in 1000 episodes in the homicidal chauffeur game . . . . . . . . . . . .

5.5 The average number of times the rules with premise $C\left(D=R_{p}\right)$ fired during 1000 episodes in game of two cars case 1 . . . . . . . . . . . 80

5.6 The average number of times the rules with premise $F\left(D=R_{p}\right)$ fired during 1000 episodes in game of two cars case $1 \ldots$. . . . . . .

5.7 The average number of times the rules with premise $V V V C$ (i.e $D=$ $R_{p}$ ) fired during 1000 episodes in game of two cars case $3 \ldots \ldots$ 
5.8 Summary of the number of episodes taken for the evader to learn its control strategy and percentage number of times the evader escapes in 1000 episodes in the game of two cars . . . . . . . . . . . . . .

5.9 Summary of the average and standard deviation of the computation times recorded during the last 100 episodes of 20 trials for each case of the homicidal chauffeur game and the game of two cars. . . . . . . . .

5.10 Real flight time (in seconds) taken for missile to intercept target with proportional navigation (at $N=2$ and $N=5$ ) and with FACL . . . . 


\section{List of Figures}

2.1 Fuzzy Logic Controller . . . . . . . . . . . . . . . . . . . . . 9

2.2 Examples of membership functions $\ldots \ldots \ldots \ldots$

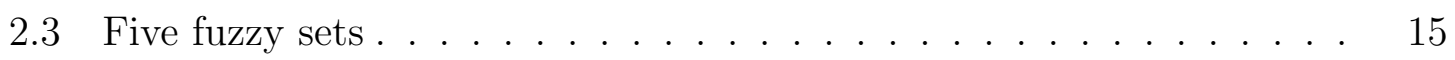

2.4 Ten fuzzy sets $\ldots \ldots \ldots \ldots \ldots \ldots \ldots \ldots \ldots \ldots$

2.5 Function $f(x)$ and its estimate $\hat{f}(x)$ using five membership functions and $\hat{f}(x)$ using ten membership functions . . . . . . . . 19

2.6 Function estimation error $f(x)-\hat{f}(x)$ using five and ten membership

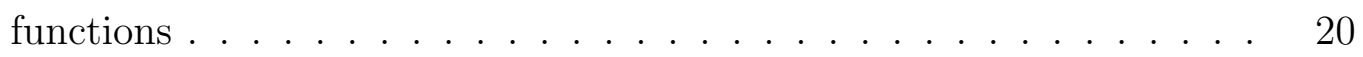

3.1 The agent-environment interaction in reinforcement learning [3] . . 22

3.2 The actor-critic architecture . . . . . . . . . . . . . . . 31

3.3 The fuzzy actor-critic learning (FACL) control structure $\ldots \ldots \ldots 35$

4.1 Homicidal chauffeur problem $[1] \ldots \ldots$. . . . . . . . . . . 42

4.2 The vehicle cannot turn into the circular region defined by its minimum turning radius $R[1] \ldots \ldots \ldots \ldots \ldots$. . . . . . . . . . . 4 44

4.3 The game of two cars $[1] \ldots \ldots \ldots \ldots \ldots$

4.4 Missile-Target Geometry . . . . . . . . . . . . . . . . . . 48

4.5 Missile-Target Geometry . . . . . . . . . . . . . . . . . . . . 49

4.6 Components for navigation . . . . . . . . . . . . . . . . . 51

5.1 Gaussian membership function of pursuer and evader before learning 60 
5.2 Gaussian membership functions of evader after learning with the QL-

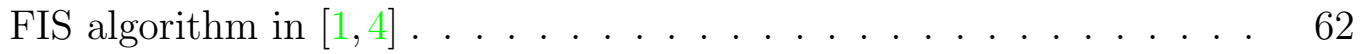

5.3 Pursuer's membership functions for all games . . . . . . . . . . 62

5.4 The evader's membership functions for Case 1 of the homicidal chauf-

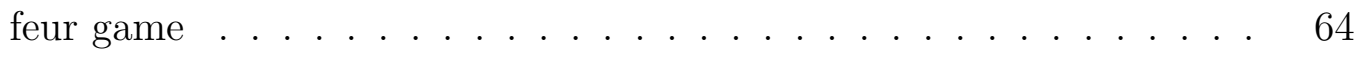

5.5 Evader increases capture time at episode 300 in the homicidal chauffeur game Case $1 \ldots \ldots \ldots \ldots \ldots \ldots$

5.6 Evader captured at episode 1000 in the homicidal chauffeur game Case 166

5.7 Critic's output parameters of rules with premise $C$ in the homicidal chauffeur game case $1 \ldots \ldots \ldots \ldots \ldots$. . . . . . . . . 6 68

5.8 The evader's membership functions for distance $D$ for the homicidal Chauffeur game case 2. Note the location of $R_{p} \ldots \ldots \ldots$

5.10 Evader escapes at episode 1000 in the homicidal chauffeur game case 271

5.11 Critic's output parameters for rules with premise $F$ in the homicidal chauffeur game case $2 \ldots \ldots \ldots \ldots \ldots \ldots \ldots \ldots \ldots$

5.9 Evader increases capture time at episode 400 in the homicidal chauffeur

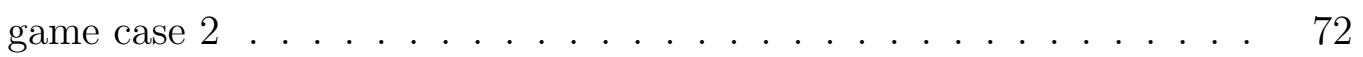

5.12 The evader's membership functions for $D$ in the homicidal chauffeur game case $3 \ldots \ldots \ldots \ldots \ldots \ldots \ldots$

5.13 Evader increases capture time at episode 100 in the homicidal chauffeur

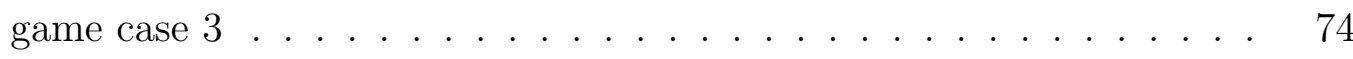

5.14 Evader escapes at episode 500 in the homicidal chauffeur game case 374

5.15 Critic's output parameters for rules with premise $V V V C$ in the homicidal chauffeur game case $3 \ldots \ldots \ldots \ldots \ldots$

5.16 Evader's average reward in the homicidal chauffeur game for case 1, case 2 and case $3 \ldots \ldots \ldots \ldots \ldots \ldots \ldots \ldots$ 
5.17 Gaussian membership functions of evader after learning . . . . . . . 78

5.18 Evader is captured at episode 1000 in game of two cars case 1 . . . 80

5.19 Critic's output parameters for rules with premise $C$ in game of two cars case $1 \ldots \ldots \ldots$. . . . . . . . . . . . . . . . . 81

5.20 Evader is captured at episode 500 in game of two cars case 2 . . . . 82

5.21 Evader is captured at episode 1000 in game of two cars case 2 . . . . 82

5.22 Critic's output parameters for rules with premise $F$ in game of two cars case $2 \ldots \ldots \ldots$. . . . . . . . . . . . . . . . . . . . . . . 83

5.23 Evader delays capture at episode 100 in game of two cars case 3 . . . 84

5.24 Evader escapes at episode 1000 in game of two cars case 3 . . . . . 85

5.25 Critic's output parameters for rules with premise $V V V C$ in game of two cars case 3 . . . . . . . . . . . . . . . . . . . . . . . . . 86

5.26 Evader's average reward in the game of two cars for case 1, case 2 and case $3 \ldots \ldots \ldots$. . . . . . . . . . . . . . . . . . 86

5.27 Missile trajectory for PN Law (at $N=2$ and $N=5$ ) and FACL(after 500 learning episodes) . . . . . . . . . . . . . . 


\section{Nomenclature}

\section{List of Abbreviations}

\begin{tabular}{|c|c|}
\hline Abbreviation & Meaning \\
\hline RL & Reinforcement Learning \\
\hline FACL & Fuzzy Actor-Critic Learning \\
\hline FIS & Fuzzy Inference System \\
\hline MDP & Markov Decision Process \\
\hline $\mathrm{MF}$ & Membership Function \\
\hline FQL & Fuzzy Q-Learning \\
\hline QLFIS & $\mathrm{Q}(\lambda)$-Learning Fuzzy Inference System \\
\hline FLC & Fuzzy Logic Controller \\
\hline DP & Dynamic Programming \\
\hline $\mathrm{TD}$ & Temporal Difference \\
\hline $\mathrm{TS}$ & Takagi-Sugeno \\
\hline $\mathrm{PN}$ & Proportional Navigation \\
\hline
\end{tabular}




\section{List of Symbols}

\begin{tabular}{|c|c|}
\hline Sybmol & Definition \\
\hline$t$ & Current time-step \\
\hline$T$ & Final time-step \\
\hline$s_{t}$ & State at time $t$ \\
\hline$s^{\prime}, s_{t+1}$ & Next state \\
\hline$a_{t}$ & Action at time $t$ \\
\hline$r_{t}$ & Reward at time $t$ \\
\hline$\mu_{i}$ & Membership degree in set $i$ \\
\hline$\gamma$ & Discount factor \\
\hline$\pi_{t}$ & Policy at time $t$ \\
\hline$S$ & Set of all states (or state space) \\
\hline$A$ & Set of all actions (or action space) \\
\hline$T()$. & Transition function \\
\hline $\mathcal{P}$ & Probability function \\
\hline$N_{t}$ & Noise \\
\hline
\end{tabular}




\section{Chapter 1}

\section{Introduction}

\subsection{Overview}

Reinforcement learning (RL) is a branch of artificial intelligence that involves an unsupervised agent learning how to take actions by interacting with its environment through trial and error [3]. The environment represents the world in which the agent exists and takes actions. When the agent takes an action, it alters the state of the environment and receives a numerical reward from the environment. The reward (which may be good or bad) informs the agent on the quality of the action taken. Repeated interaction with the environment enables the agent to learn what actions produce good rewards. The goal of the agent then becomes to maximize the cumulative reward for the duration of its interaction with the environment [3]. RL methods generally apply to problems with small discrete state-action spaces. In order to apply RL to problems involving very large discrete or continuous state-action spaces, a good function approximator such as a Neural network or a fuzzy inference system (FIS) is required $[5,6]$.

A fuzzy inference system (FIS) uses fuzzy logic theory to imitate human intuition 
and reasoning. In the literature, fuzzy systems have proven to be useful in modeling complex situations whose behaviours are not fully understood and in situations where approximate solutions are sufficient $[7,8]$. Fuzzy systems approximate system behavior that are uncertain and processes where analytic or numerical solution do not exist [9]. Fuzzy inference systems have been present and successful in real-life applications like automobile transmission control, temperature control, washing machines etc. An FIS can be used to model complex processes in continuous time domain such as in differential games which were introduced by Rufus Isaacs [2]

Differential games are continuous time games which usually require differential equations to define the dynamics of the agents involved. A differential game in simple

form involves two or more agents with conflicting goals [2]. There are many types of differential games but a common subset of differential games is the pursuit-evasion differential game which involves a pursuer and an evader [2]. In a pursuit-evasion game, the goal of the pursuer is to capture the evader while the goal of the evader is to escape from the pursuer. Pursuit-evasion games can be applied to numerous combat scenarios such as the movement of ships, aircraft, satellites or missiles [2].

\subsection{Literature Review}

Reinforcement learning (RL) primarily involves an agent interacting with its dynamic environment and finding a good policy of state-action pairs that maximizes a reward or reinforcement [3]. A good way to formalize RL problems is as Markov decision processes(MDP). A Markov decision process is a mathematical representation of a decision making process. In an MDP, the learning agent at any time $t$ is said to be in a state $s$ and takes an action $a$. As a consequence of the action taken, the agent transitions to the next state $s^{\prime}$ and receives a scalar reward $r$. The purpose of RL is 
find good sequence of state-action pairs that maximizes the expected sum of future rewards $r$ for all states [3]. Reinforcement learning is a very popular learning technique for adaptive agents because an explicit model of the environment is generally not required.

Several RL methods have been studied thoroughly over the years [3,10-12]. The $Q$-learning algorithm, which was proposed by Watkins [13] and actor-critic methods are two types of RL techniques that have been widely used. Reinforcement learning algorithms like those aforementioned are required to store estimates of expected future rewards for every state or state-action pair. Therefore, they generally only apply to problems with small discrete state-action spaces [3,14]. For problems with large discrete state-action spaces or continuous state-action spaces, some form of function approximation has to be used. Differential games are good examples of problems that require continuous state-action spaces.

A pursuit-evasion game is a type of differential game. In a pursuit-evasion game, a pursuer endeavours to capture an evader in minimal time while the evader aims to escape or maximize the capture time $[2,15]$. Two popular examples of pursuit-evasion games are; the homicidal chauffeur game and the game of two cars. In the homicidal chauffeur game $[2,16]$, the pursuer which is modeled as a car is determined to capture an agile and more maneuverable evader. However, in the game of two cars $[2,16]$, both pursuer and evader are modeled as cars. Time optimal strategies for both pursuer and evader to achieve their objectives have been studied in [2], [17] and [18]. Various research also show that the players in pursuit-evasion games can successfully learn their control strategy using RL techniques $[4,19]$. The concept of RL in pursuitevasion games can be applied to various real-world situations like autonomous air vessels, water vessels, guided missile navigation, etc. In order to apply RL to pursuitevasion games, researchers have combined RL methods with fuzzy inference systems. 
Fuzzy inference systems (FIS) have proven to be good function approximators in problems that are complex or imprecise [20].

A fuzzy inference system makes use of fuzzy logic to categorize inputs into linguistic variables and map those inputs to outputs in a way that is representative of human intuition and reasoning [21,22]. The input space of the FIS is partitioned into fuzzy sets or membership functions (MFs) which have linguistic labels. There are several types of MF partitions. Two commonly used MFS are the triangular MF and Gaussian MF [23]. Different choices of MFs have been found to have insignificant effects on inference results if the MFs are properly fine-tuned [20]. Lionel Jouffe [6] introduced two fuzzy RL methods namely, fuzzy-actor critic learning (FACL) and fuzzy $Q$ - learning (FQL). In the literature, RL techniques have been applied in pursuitevasion games to train both pursuer and evader to learn their optimal strategies. The authors in [4] proposed a $Q(\lambda)$-learning fuzzy inference system (QLFIS) which successfully trained the pursuer to learn its optimal control strategy in the game of two cars. In [4], an FIS is used to represent the state and action spaces of the pursuer and $Q$ - learning in combination with eligibility traces is used to tune the input and output parameters of the FIS.

The QLFIS algorithm in [4], was applied to train only the pursuer to learn its optimal strategy in the game of two cars. To the best of our knowledge, only the research done in [1] has investigated the use of RL in training the evader to learn its strategy in pursuit-evasion games. The study carried out in [1] applies the QLFIS algorithm to train both the pursuer and the evader to learn their control strategies in the homicidal chauffeur game and the game of two cars. The FIS structure in [1] consists of Gaussian MFs, also both input and output parameters of the FIS were tuned using the QLFIS algorithm.

In our approach, we use the FACL algorithm to train both the pursuer and evader 
in the homicidal chauffeur game and the game of two cars. Our main focus is the evader learning its time optimal control strategy. We investigate whether the evader will learn just as well as in [1] or even better. We investigate how to minimize computation time by using only triangular membership functions and by updating only the output parameter of the fuzzy inference system.

\subsection{Contributions and Thesis Organization}

To the best of our knowledge, only the research carried out by my colleague in [1] investigates how an evader and a pursuer can learn their control strategy using reinforcement learning methods. In [1], QLFIS algorithm was used to train both pursuer and evader to learn their control strategies. Both input and output parameters of the fuzzy inference system were tuned in $[1,4]$. In this thesis, we investigate how the pursuer and evader can learn their control strategies in the homicidal chauffeur game and the game of two cars using the FACL algorithm. The major contributions of this thesis are as follows

- We train the pursuer and evader to learn their control strategies using the FACL algorithm in the homicidal chauffeur game and the game of two cars. We focus more on the results of the evader.

- We use triangular membership functions over the inputs of the FIS instead of Gaussian MFs that are used in $[1,4]$ to reduce computation time.

- We selected different inputs to the evader's FIS that are different from the inputs used in $[1,4]$.

- To further,reduce computation time, we tune only the output parameters of the FIS as opposed to the approach used in $[1,4]$ whereby both input and output 
parameters of the FIS are tuned.

- Although our main focus was the evader learning its optimal strategy, the pursuer successfully learned its strategy using only one input state in its FIS.

- We introduce the FACL algorithm to the inertial homing missile guidance problem where the parameter to be controlled is a normal force rather than a steering angle.

The remainder of this thesis is organized as follows:

- Chapter 2 presents information on fuzzy sets and fuzzy logic systems. We introduce the two common types of fuzzy inference methods: the Mamdani and the Takagi-Sugeno fuzzy inference methods. We discuss the function approximation property of fuzzy inference systems.

- Chapter 3 introduces reinforcement learning, Markov decision processes, dynamic programming and temporal difference learning. It also introduces two fuzzy reinforcement learning techniques: The fuzzy actor-critic learning and $\mathrm{Q}(\lambda)$-learning for continuous state and action spaces.

- Chapter 4 discusses pursuit-evasion differential games especially the two common types namely: the homicidal chauffeur game and the game of two cars. It also introduces the inertial homing missile guidance problem which may also be classified as a type of differential game.

- Chapter 5 describes the fuzzy actor-critic learning (FACL) algorithm that will be used to train the evader in homicidal chauffeur game and the game of two cars. We compare our results with results observed when using the $\mathrm{Q}(\lambda)$-learning fuzzy inference system to train the evader (in [1]) to learn its control strategy. 
The FACL algorithm is also used to train the missile in the inertial homing missile guidance problem. We compare the results when using FACL versus when using the proportional navigation guidance law to guide the missile.

- Chapter 6 concludes this thesis by stating the main contributions and points out the future research directions. 


\section{Chapter 2}

\section{Fuzzy Control Systems}

\subsection{Fuzzy Logic Control}

Fuzzy systems, introduced by Lotfi Zadeh in [24], are approximation function systems that are capable of modelling real world problems and mimicking human intuition to solve those problems. Applications of fuzzy systems range from small examples like product survey questions; where customers are asked to choose exactly to what degree they are satisfied or dissatisfied with some service or product to advanced applications in industrial manufacturing, automatic control, automobile production, banks, hospitals etc.

Fuzzy logic controllers (FLCs) also known as fuzzy inference systems (FIS), depicted in figure 2.1, are expert systems based on If-Then logic rules designed to map premises to conclusions that are expressed as linguistic labels known as fuzzy sets [23]. Implementing fuzzy logic control techniques to real world applications requires the following steps, namely:

- Fuzzification

- Fuzzy Inference Process 


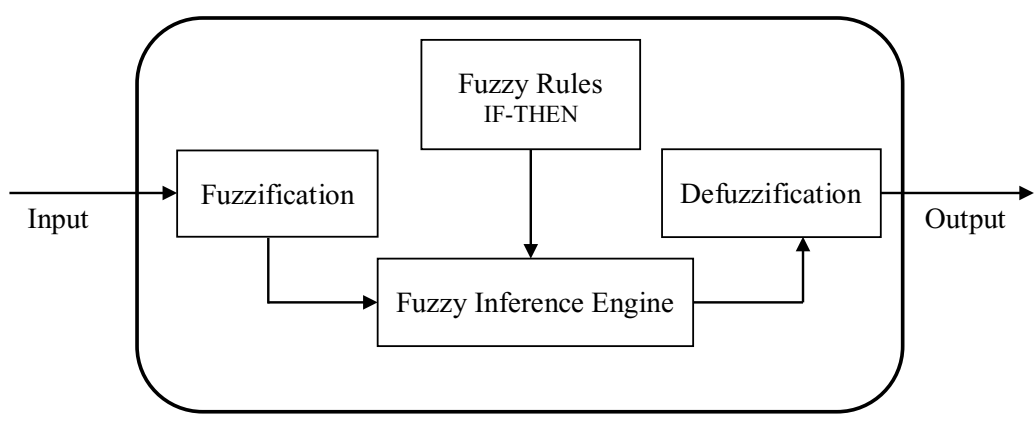

Figure 2.1: Fuzzy Logic Controller

- Defuzzification

\section{$2.2 \quad$ Fuzzy Sets and Operations}

Fuzzy sets introduced by [25] is an extension of the notion of classical (crisp) sets. In a crisp set, the membership of an element in the set is binary. That is, the element either fully belongs to the set (with a degree of 1 ) or it completely does not (with a degree of 0 ). However, in fuzzy sets, elements are allowed to have partial membership in a set. Each element is assigned a degree of membership in a set. This membership value can be any number in the real unit interval $[0,1]$. To illustrate, let's define two classic sets $A$ and $B$ containing four elements and three elements respectively, along with a universal set, $F$, with ten elements:

$$
\begin{aligned}
F & =\left\{x_{1}, x_{2}, x_{3}, x_{4}, x_{5}, x_{6}, x_{7}, x_{8}, x_{9}, x_{10}\right\} \\
A & =\left\{x_{1}, x_{2}, x_{3}, x_{4}\right\} \\
B & =\left\{x_{1}, x_{2}, x_{3}\right\}
\end{aligned}
$$


Applying the classical set reasoning to equation 2.1, the element $x_{4}$ in the universal set fully belongs to set $A$ with a membership degree of 1 . We denote the degree to which $x_{4}$ has membership in set $A$ as $\mu_{A}\left(x_{4}\right)=1$. At the same time, the element $x_{4}$ does not belong to set $B$ with membership degree $\mu_{B}\left(x_{4}\right)=0$. Where $\mu_{i}$ represents the degree of membership in the set $i$. Fuzzy sets on the other hand are generally expressed in this form

$$
C=\left\{\left(x, \mu_{C}(x)\right) \mid x \in F\right\}
$$

where, $\mu_{C}(x)$ is the membership degree to which x belongs to a fuzzy set $C$ in the real range $[0,1]$. An example is shown below

$$
C=\left\{\left(x_{7}, 0.2\right),\left(x_{8}, 0.89\right),\left(x_{9}, 0.5\right)\right\}
$$

where we can say the element $x_{9}$ belongs to set $C$ to a degree of 0.5 .

Another major difference between fuzzy sets and classical sets is the interpretation of set operations such as union and intersection operations. This difference is due to the graded property of fuzzy sets. Intersection operation of two fuzzy sets is generally carried out using a binary function called the triangular norm ( $t$-norm) operator. Let's assume we have two fuzzy sets $A$ and $B$ defined in the same universe of discourse $U$, the intersection of these two fuzzy sets is a fuzzy set whose membership function is

$$
\mu_{A \cap B}(x)=T\left(\mu_{A}(x), \mu_{B}(x)\right)=\mu_{A}(x) \star \mu_{B}(x)
$$

where $\star$ is defined as the t-norm operator. Two commonly used t-norm operators are

$$
\begin{aligned}
& \text { Minimum }: T_{\min }(a, b)=\min (a, b) \\
& \text { Algebraic product }: T_{a p}(a, b)=a b .
\end{aligned}
$$


Similarly, the union of two fuzzy sets is generally carried out using a binary function called the triangular conorm (s-norm) operator. The union of the fuzzy sets $A$ and $B$ gives a fuzzy set whose membership function is:

$$
\mu_{A \cup B}(x)=S\left(\mu_{A}(x), \mu_{B}(x)\right)=\mu_{A}(x) \dot{+} \mu_{B}(x)
$$

where $\dot{+}$ is defined as the s-norm operator. Two commonly used s-norm operators are

$$
\begin{aligned}
& \text { Maximum : } S_{m a x}(a, b)=\max (a, b) \\
& \text { Algebraic sum : } S_{a p}(a, b)=a+b-a b .
\end{aligned}
$$

The fuzzification process involves transforming classical or crisp (real-valued) input data into linguistic labels represented by fuzzy sets. This is achieved by first creating membership functions over the input (and sometimes output) space to determine the degree of membership.

Membership functions (MFs) are curves that allow us to graphically represent a fuzzy set and define how to map a point in the input space to a membership degree in the range $[0,1]$. Figure 2.2 shows various types of membership functions commonly used in fuzzifying real-valued input data. The Gaussian and triangular MFs are two of the most commonly used MFs.

\subsubsection{Fuzzy Inference Process}

The Fuzzy Inference process involves using fuzzy control rules to map the fuzzified input data to the output [21]. The fuzzy control rules can be likened to human intuition or knowledge of an expert in the field of application. The control rules are 



Figure 2.2: Examples of membership functions

modeled using a sequence of fuzzy If-Then statements used in describing what output or action(s) should be taken in terms of the currently perceived input. The rules are defined as:

$$
\Re_{l}: I F x \text { is } A \text { THEN } y \text { is } B
$$

where $A$ and $B$ are fuzzy sets defined on the universe of discourse $X$ and $Y$ respectively. From equation 2.10, the term " $x$ is $A$ " is the antecedent or premise while " $y$ is $B$ " is the conclusion or consequence [23]. The fuzzy inference engine in figure 2.1, is used in combination with the fuzzy If-Then rules in the rule base to map the inputs to the outputs. The two most commonly used fuzzy types of inference methods are: the Mamdami and Takagi-Sugeno fuzzy inference methods.

The Mamdami fuzzy inference method was proposed in 1975 by Ebrahim Mamdani [26] with the aim of controlling a steam engine and boiler combination by synthesizing a set of linguistic control rules obtained from experienced human operators. The unique thing about this method is in fuzzifying the output values to produce membership functions that can be aggregated with the rules to produce crisp values in the defuzzification process. The method usually involves applying the minimum 
method in equation 2.5 for all t-norm fuzzy operations and the maximum method in equation 2.8 is used for all s-norm fuzzy operations.

The Takagi-Sugeno fuzzy inference method [27] is similar to the Mamdami method in many ways. The first two operations (fuzzifying the inputs and applying fuzzy operators) are the same. The only difference is that the Takagi-Sugeno fuzzy inference method maps the input fuzzy sets to a crisp output function. The commonly used fuzzy operators in the Takagi-Sugeno fuzzy inference method include: the algebraic product method in equation 2.6 for all $t$-norm operations and the maximum method in equation 2.8 for all $s$-norm operations.

\subsubsection{Defuzzification}

To make the output of a fuzzy inference system useful in real applications, a defuzzification process is needed. Defuzzification is the conversion of a fuzzy output (linguistic variable) to classical or crisp values. Some known defuzzification methods include: the mean of maximum method, the height method, the center of gravity method, the weighted average method etc.

In this thesis, we make use of the weighted average defuzzification method which yields a crisp output $y$ expressed as $[22,28]$

$$
y=\frac{\sum_{l=1}^{L}\left(\prod_{j=1}^{J} \mu_{A_{j}^{l}}\left(x_{j}\right)\right) f^{l}}{\sum_{l=1}^{L}\left(\prod_{j=1}^{J} \mu_{A_{j}^{l}}(x)\right)}
$$

where $J$ is the number of inputs, $L$ is the number of rules and $f^{l}$ is the constant conclusion for each rule $l$. 


\subsubsection{Function Approximation using Fuzzy Logic}

To illustrate the function approximation property of fuzzy systems, we consider an example taken from [28].

\subsubsection{Example 1}

Given a nonlinear system described by the function

$$
f(x)=\frac{1-\exp ^{-x(t)}}{1+\exp ^{-x(t)}}
$$

We find the estimate of the function $f(x)$ using a zero-order Takagi-Sugeno fuzzy inference system for different values of $x$. First, we fuzzify the inputs by creating membership functions over the input space $x$. We create five triangular membership functions evenly spread over the interval $x \in[-3,3]$ with linguistic terms: negative medium (NM), negative small (NS), zero (ZE), positive small (PS), and positive medium (PM). The equation for each triangular membership function is given as [23]

$$
\mu_{A}\left(x_{i}\right)= \begin{cases}0, & x \leq a \\ \frac{x-a}{b-a}, & a \leq x \leq b \\ \frac{c-x}{c-b}, & b \leq x \leq c \\ 0, & c \leq x\end{cases}
$$

where $a, b$ and $c$ are the lower limit, peak and upper limit of the triangular membership function respectively. Figure 2.3 shows the structure of the five membership functions. Each membership function in Figure 2.3 overlaps with only one membership function on both sides. This overlap ensures that for any input $x \in[-3,3]$, only a maximum 


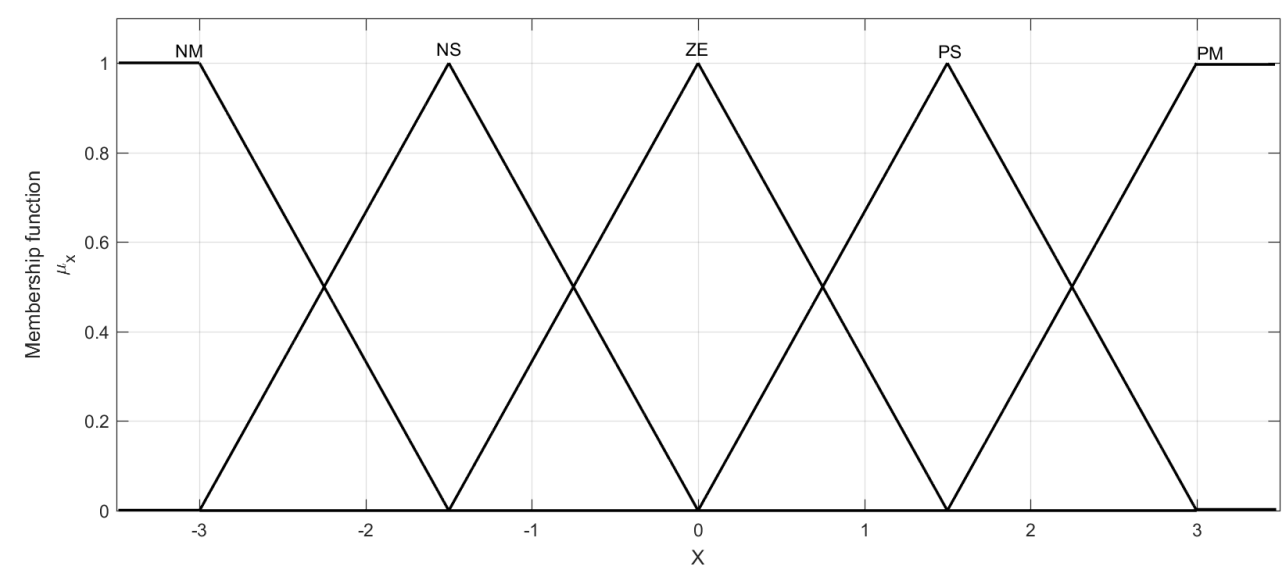

Figure 2.3: Five fuzzy sets

of two rules will activate or fire and all the other rules will be zero. We also note from Figure 2.3 that all membership functions overlap at $\mu_{x}=0.5$. This means that the membership degrees $\left(\mu_{x}\right)$ of the rules that fire will always sum up to unity $(1)$, making the denominator of Equation 2.11 unity regardless of the number of inputs. The amount of computation will not be impacted even in applications with more than one input. The If-Then rules for the fuzzy inference system are

$$
\begin{aligned}
& \Re_{l}: \text { IF } x \text { is }-1.5 \text { THEN } f(x) \text { is }-0.64 \\
& \Re_{2}: \text { IF } x \text { is }-0.5 \text { THEN } f(x) \text { is }-0.24 \\
& \Re_{3}: \text { IF } x \text { is } 0 \text { THEN } f(x) \text { is } 0.00 \\
& \Re_{4}: \text { IF } x \text { is } 0.5 \text { THEN } f(x) \text { is } 0.24 \\
& \Re_{5}: \text { IF } x \text { is } 1.5 \text { THEN } f(x) \text { is } 0.64
\end{aligned}
$$

where the conclusions for each rule are $f^{1}=-0.64, f^{2}=-0.24, f^{3}=0.00, f^{4}=$ $0.24, f^{5}=0.64$

To compute the estimate of the nonlinear function given in Equation 2.12, we use 
the defuzzication method from Equation 2.11

$$
\begin{aligned}
\hat{f}(x)= & \frac{\sum_{l=1}^{5}\left(\mu_{A^{l}}(x)\right) f^{l}}{\sum_{l=1}^{5}\left(\mu_{A^{l}}(x)\right)} \\
= & \frac{-0.64 \mu_{N M}(x)-0.24 \mu_{N S}(x)+0.24 \mu_{P S}(x)+0.64 \mu_{P M}(x)}{\mu_{N M}(x)+\mu_{N S}(x)+\mu_{Z E}(x)+\mu_{P S}(x)+\mu_{P M}(x)} \\
= & \frac{-0.64 \mu_{N M}(x)-0.24 \mu_{N S}(x)+0.24 \mu_{P S}(x)+0.64 \mu_{P M}(x)}{1}
\end{aligned}
$$

The estimated function $\hat{f}(x)$ using five triangular membership functions is shown in Figure 2.5 alongside the function $f(x)$. The estimation error $f(x)-\hat{f}(x)$ is illustrated in Figure 2.6 and shows that the value of the maximum absolute error $|f(x)-\hat{f}(x)|<0.45$. We know that this performance can be improved by using more membership functions over the universe of discourse. For example, if we increase the number of membership functions to ten evenly spread over the interval $[-3,3]$ in Figure 2.4. The linguistic terms are: negative huge $(\mathrm{NH})$, negative large (NL), negative big (NB), negative medium (NM), negative small (NS), positive small (PS), positive medium (PM), positive medium (PM), positive large (PL) and positive huge (PH). The fuzzy IF-THEN rules are given in 2.16 


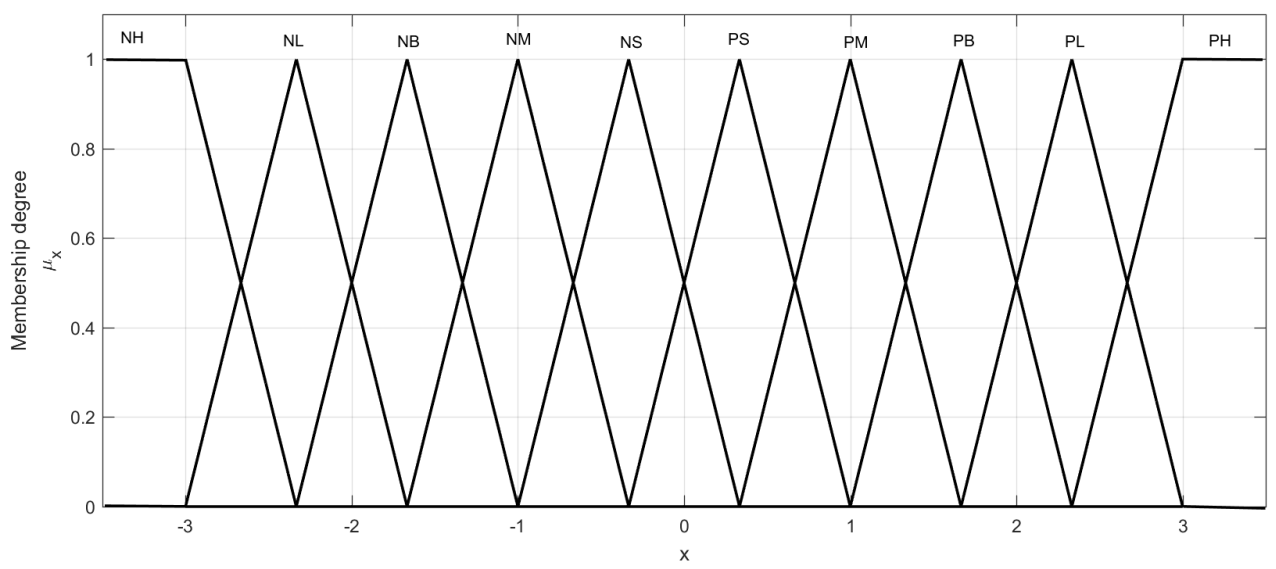

Figure 2.4: Ten fuzzy sets

$\Re_{1}:$ IF $x$ is -3.0 THEN $f(x)$ is -0.91

$\Re_{2}:$ IF $x$ is $-2.5 T H E N f(x)$ is -0.85

$\Re_{3}:$ IF $x$ is -1.5 THEN $f(x)$ is -0.64

$\Re_{4}:$ IF $x$ is -1.0 THEN $f(x)$ is -0.46

$\Re_{5}:$ IF $x$ is -0.5 THEN $f(x)$ is -0.24

$\Re_{6}:$ IF $x$ is 0.5 THEN $f(x)$ is 0.24

$\Re_{7}:$ IF $x$ is 1.0 THEN $f(x)$ is 0.46

$\Re_{8}$ : IF $x$ is 1.5 THEN $f(x)$ is 0.64

$\Re_{9}:$ IF $x$ is 2.5 THEN $f(x)$ is 0.85

$\Re_{10}:$ IF $x$ is 3.0 THEN $f(x)$ is 0.91

where the conclusions for each rule is set as $f^{1}=-0.91, f^{2}=-0.85, f^{3}=-0.64, f^{4}=$ $-0.46, f^{5}=-0.24, f^{6}=0.24, f^{7}=0.46, f^{8}=0.64, f^{9}=0.85, f^{1} 0=0.91$ 
Equation 2.11 becomes

$$
\begin{aligned}
\hat{f}(x)= & \frac{\sum_{l=1}^{10}\left(\mu_{A^{l}}(x)\right) f^{l}}{\sum_{l=1}^{10}\left(\mu_{A^{l}}(x)\right)} \\
& -0.91 \mu_{N H}(x)-0.85 \mu_{N L}(x)-0.64 \mu_{N B}(x)-0.46 \mu_{N M}(x)-0.24 \mu_{N S}(x) \\
= & \frac{+0.24 \mu_{P S}(x)+0.46 \mu_{P M}(x)+0.64 \mu_{P B}(x)+0.85 \mu_{P L}(x)+0.91 \mu_{P H}(x)}{1}
\end{aligned}
$$

The estimated function $\hat{f}(x)$, using ten triangular membership functions, is depicted in Figure 2.5. The estimation error $f(x)-\hat{f}(x)$ illustrated in Fig.2.6 shows that the value of the maximum absolute error $|f(x)-\hat{f}(x)|<0.20$ which is more accurate than when we used five membership functions.

We observed that increasing the number of membership functions from five to ten significantly improved the estimation of the function in Equation 2.12. In this thesis, we will apply a fuzzy inference system to approximate the state and action space of one or more learning agents. Also, reinforcement learning algorithms will be used to tune the outputs of fuzzy inference systems. We will use this idea of increasing the number of membership functions to better approximate the agent's actions. 


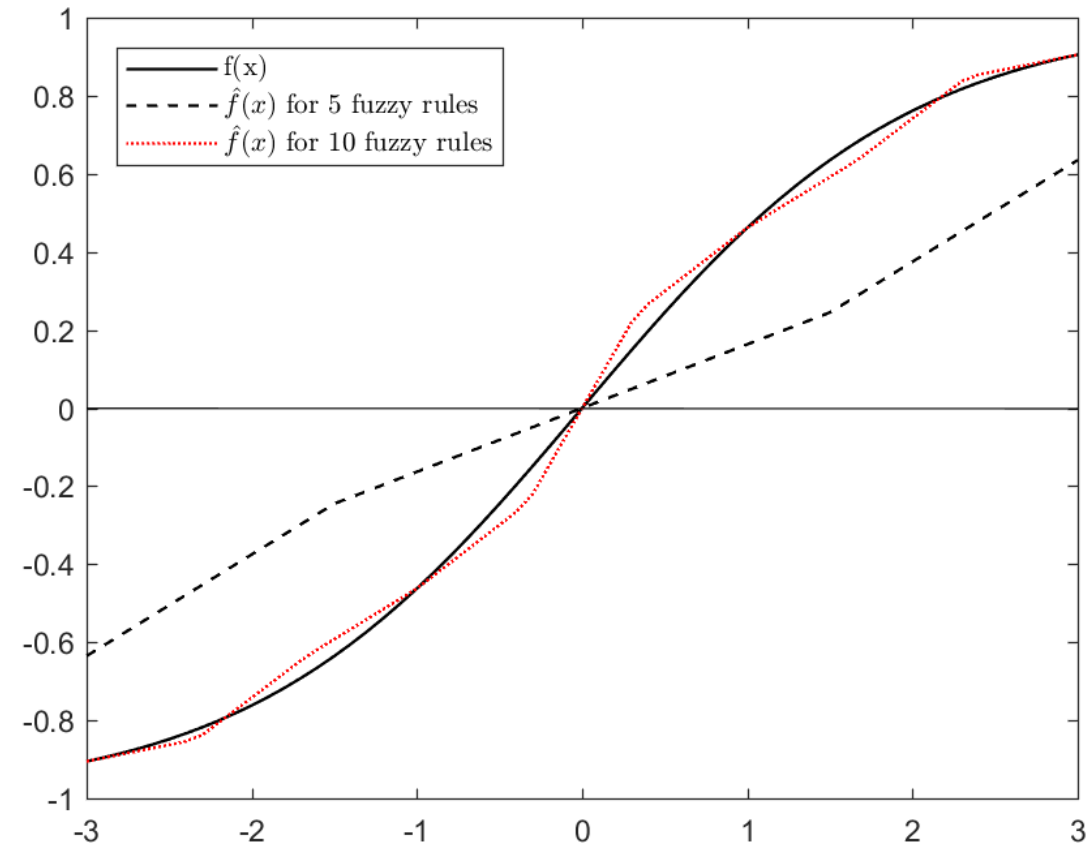

Figure 2.5: Function $f(x)$ and its estimate $\hat{f}(x)$ using five membership functions and $\hat{f}(x)$ using ten membership functions 


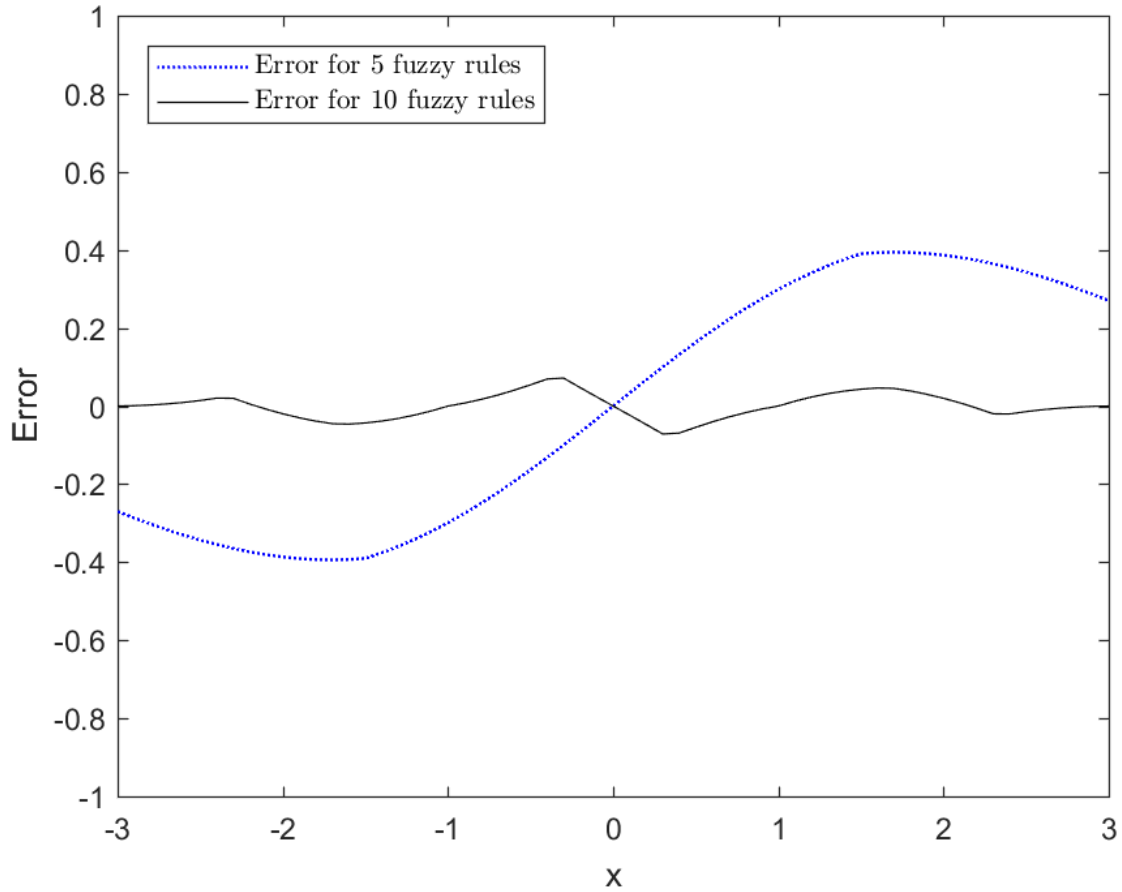

Figure 2.6: Function estimation error $f(x)-\hat{f}(x)$ using five and ten membership functions 


\section{Chapter 3}

\section{Reinforcement Learning}

\subsection{Introduction}

Reinforcement learning (RL) is a computational approach to learning the correct actions to take in order to maximize a numerical reward [3]. In a reinforcement learning problem, the learner (or agent) has no prior knowledge of the appropriate actions to take in each situation (or state) leading up to the fulfillment of a predefined goal. The agent must learn which actions would produce the most reward by repeated trial and interaction with its environment. The reward received by the agent tells it how good or bad its selected action was. The agent's actions may not only affect the immediate reward but the next state and all succeeding rewards as well [3].

Unlike supervised learning, where an agent is equipped with a dataset that characterizes the environment and a mapping of actions to states. An RL agent has no supervisor and is required to learn from its own experience by interacting with its environment. 


\subsubsection{Elements of reinforcement learning}

The setup of a reinforcement learning problem usually consists of an agent and the environment in which it exists. The agent is the learner that is able to take actions within the environment. The environment serves as the outside world to the agent and provides the agent with a reward and information about its next state. Figure 3.1 depicts the interaction model between the learning agent and the environment [3].

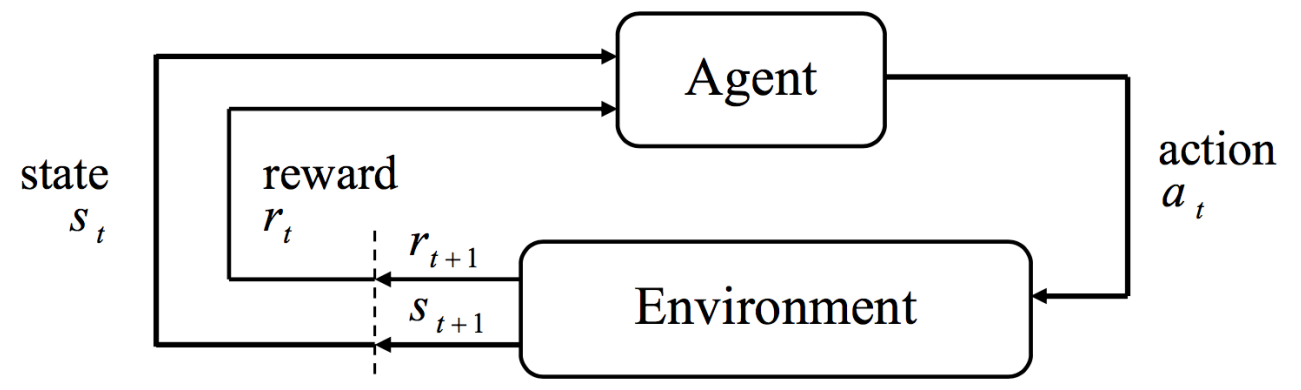

Figure 3.1: The agent-environment interaction in reinforcement learning [3]

Let's define a sequence of discrete time steps $\{t=1,2, \ldots, T\}, a_{t}$ is the action taken at time step $t, s_{t}$ is the state perceived by the learning agent from the environment and $r_{t} \in \mathbb{R}$ is the numerical reward at time $t$. At each time step $t$, the learning agent is presented with a state $s_{t}$ and takes an action $a_{t}$. As a consequence of this action, the agent receives a numerical reward and moves on the next state $s_{t+1}$. The agent's goal is to maximize the total rewards received from the next state and all subsequent states.

The reward in a reinforcement learning problem is a simple number $r_{t} \in \Re$. The agent receives a sequence of immediate rewards $r_{t+1}, r_{t+2}, r_{t+3}, \ldots$, after each time step $t$. The sum of the expected future rewards at time $t$ is given as

$$
R_{t}=r_{t+1}+r_{t+2}+r_{t+3}+\cdots r_{T}
$$


where $T$ is a final time step in an episode. An episode starts from an initial state and ends at a terminal state. Tasks which can be finished in a finite time are called episodic tasks while tasks that go on indefinitely are called continuing tasks [3].

If we introduce the notion of discounting rewards, the goal of the agent becomes to maximize the sum of the discounted future rewards written as

$$
R_{t}=r_{t+1}+\gamma r_{t+2}+\gamma^{2} r_{t+3}+\cdots=\sum_{k=0}^{\infty} \gamma^{k} r_{t+k+1}
$$

where $\gamma \in[0,1]$ is the discount factor. The discount factor determines the current value of future rewards such that a reward received $k$ time steps in the future is only worth $\gamma^{k-1}$ times of the value in the current time step. If $\gamma<1$, the infinite sum in equation 3.2 has a finite value as long as the reward sequence $r_{k}$ is bounded. If $\gamma=0$, the agent always prioritizes maximizing its immediate reward. If the discount factor $\gamma$ approaches 1 , the agent prioritizes future rewards.

Apart from the agent, environment and reward, three other important elements of a reinforcement learning problem worthy of note are: a policy, a value function and optionally, a model of the environment.

An agent's policy $\pi_{t}$, is defined as the mapping from states to probabilities of choosing each possible action [3]. We use $\pi_{t}(s, a)$ to denote a policy with the probability of choosing action $a_{t}=a$ at the state $s_{t}=s$. A policy defines the agent's behaviour at a given time. A policy is said to be an optimal policy if the agent can maximize its accumulated received rewards following this policy. Reinforcement learning methods specify how the agent changes its policy as a result of its experience to maximize the total amount of reward it receives over the long run [3].

Although, the reward signal indicates what is good in an immediate sense, a value function represents what is good in the long run. Basically, the value of a state is the 
total amount of reward an agent can expect to accumulate over the future, starting from the current state and following a given policy. Unlike rewards, values indicate the long-term desirability of states after taking into account the states that are likely to follow, and the rewards available in those states [3]. For example, a state might always yield a low immediate reward but still have a high value because it is regularly followed by other states that yield high rewards.

\subsection{Markov Decision Processes}

A reinforcement learning (RL) problem can be formalized as a Markov decision process (MDP) [3]. An MDP describes the environment of the agent. The agent makes decisions based on the state of the environment. For an RL problem to be described as a Markov decision process, it has to satisfy the Markov property. The Markov property is said to be satisfied if the current state $s_{t}$ contains all the relevant information necessary to take an action $a_{t}$ and transition into the next state $s_{t+1}$ [3]. Once the current state in known, the history may be discarded, and that state has enough information to give us the same description of the future as if we have all the history. The Markov property can be written as [3]

$$
\mathcal{P}\left[S_{t+1}=s^{\prime} \mid S_{t}, a_{t}\right] \quad \forall s, s^{\prime} \in S, a \in A
$$

An MDP can be defined as a tuple [28], [29]

$$
\{S, A, R, T, \gamma\}
$$

where $S$ is the state space, $A$ is the action space, $R: S \times A \times S \rightarrow \mathbb{R}$ is the reward function, $T: S \times A \times S \rightarrow[0,1]$ is the transition function and $\gamma \in[0,1]$ is the discount 
factor. The transition function $T\left(s, a, s^{\prime}\right)$, which will also be written as $T_{s s^{\prime}}^{a}$, denotes the probability of moving to the next state $s^{\prime}$ given the current state $s$ and action $a$ such that

$$
\sum_{s^{\prime} \in S} T\left(s, a, s^{\prime}\right)=1 \quad \forall s \in S, \forall a \in A
$$

The reward function $R_{s s^{\prime}}^{a}$ is defined as the expected reward value to be received at the next state $s^{\prime}$ given the current action $a$ and the current state $s$. This is expressed in the form [3]

$$
R_{s s^{\prime}}^{a}=\mathbb{E}\left[R_{t+1} \mid S_{t}=s, A_{t}=a, S_{t+1}=s^{\prime}\right]
$$

Recall from subsection 3.1.1 that an agent's policy is a mapping from states to actions. A policy, denoted by $\pi(s, a)$ satisfies

$$
\sum_{a \in A} \pi(s, a)=1 \quad \forall s \in S
$$

Since the goal of an agent in an MDP is to maximize the expected reward in the long term, there exists an optimal policy that achieve this goal. We denote $\pi^{*}(s, a)$ as the optimal policy for the agent. The value of a state (or state-value function) $V^{\pi}(s)$ is defined as the expected received reward when the player follows a policy $\pi$ at state $s$ and thereafter [3]. The state-value function $V^{\pi}(s)$ is given as

$$
V^{\pi}(s)=E_{\pi}\left\{\sum_{k=0}^{\infty} \gamma^{k} r_{k+t+1} \mid s_{t}=s\right\}
$$

where $t$ is the current time step, $\gamma \in[0,1]$ is a discount factor, and $r_{k+t+1}$ is the received immediate reward at time step $k+t+1$. For an optimal policy $\pi^{*}$, the state-value function satisfies

$$
V^{*}(s) \geq V^{\pi}(s) \quad \forall \pi, \forall s \in S
$$


The state-value function in (3.8) can also be written as a recursive equation known as the Bellman equation [3] expressed as follows

$$
V^{\pi}(s)=\sum_{a \in A} \pi(s, a) \sum_{s^{\prime} \in S} T\left(s, a, s^{\prime}\right)\left(R\left(s, a, s^{\prime}\right)+\gamma V^{\pi}\left(s^{\prime}\right)\right)
$$

The optimal state-value function $V^{*}(s)$, also called the Bellman optimality equation, can be expressed as

$$
V^{*}(s)=\max _{a \in A} \sum_{s^{\prime} \in S} T\left(s, a, s^{\prime}\right)\left(R\left(s, a, s^{\prime}\right)+\gamma V^{*}\left(s^{\prime}\right)\right)
$$

The action-value function $Q^{\pi}(s, a)$ is defined as the expected reward of choosing an action $a$ at state $s$ and following a policy $\pi$ thereafter. The action-value function $Q^{\pi}(s, a)$ is given as

$$
Q^{\pi}(s, a)=\sum_{s^{\prime} \in S} T_{s s^{\prime}}^{a}\left(R_{s s^{\prime}}^{a}+\gamma V^{\pi}\left(s^{\prime}\right)\right)
$$

Similar to the state-value function, there also exists an optimal action-value function $Q^{*}(s, a)$ if the agent chooses an action $a$ at state $s$ and consequently follows the optimal policy $\pi^{*}$. The optimal action-value function is expressed as

$$
Q^{*}(s, a)=\sum_{s^{\prime} \in S} T_{s s^{\prime}}^{a}\left(R_{s s^{\prime}}^{a}+\gamma V^{*}\left(s^{\prime}\right)\right)
$$

\subsubsection{Dynamic Programming}

Dynamic programming (DP) methods are a class of algorithms that can be used to compute optimal policies given a perfect model of the environment as a Markov decision process [3]. A perfect model of the environment is a model that can perfectly predict or mimic the behavior of the environment [3]. We can not assume that a model will be available for all applications. However, DP methods are very important 
because they define the computational structure which can be used when no model is available [11]. To obtain a model of the environment, one needs to know the agent's reward function and transition function in an MDP.

The main idea behind DP is in the use of value functions to find the agent's optimal policy. One method for finding the optimal policy is called the value iteration algorithm which is listed in Algorithm 1. The value iteration is derived by converting the Bellman optimality equation (3.11) into an update rule. The value function will theoretically converge to the optimal value function as the iteration goes to infinity. But in practice, as shown in Algorithm 1, we terminate the value iteration when the value function converges within a small range $[-\sigma, \sigma]$. Then, we update the agent's policy based on the updated value function.

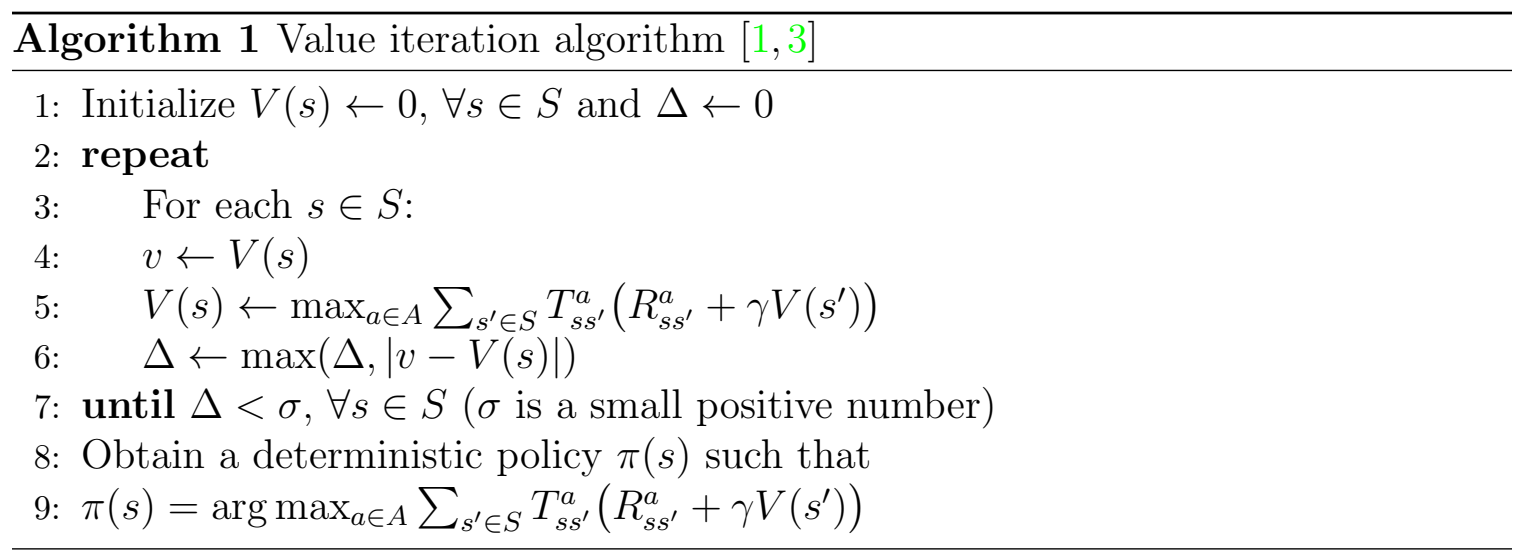

\subsection{Temporal-Difference Learning}

Temporal-difference (TD) learning methods learn directly from new experiences and do not require the model of the environment [3]. The two main temporal-difference learning algorithms are Q-learning and actor-critic learning [9]. TD methods can be used to estimate the state-value function $V^{\pi}$ for a given policy $\pi$. We refer to 
Equation 3.8 and write it in the form

$$
\begin{gathered}
V^{\pi}(s)=E_{\pi}\left\{r_{t+1}+\sum_{k=1}^{\infty} \gamma^{k} r_{k+t+1} \mid s_{t}=s\right\} \\
V^{\pi}(s)=E_{\pi}\left\{r_{t+1}+\gamma \sum_{k=0}^{\infty} \gamma^{k} r_{k+t+2} \mid s_{t}=s\right\} \\
V^{\pi}(s)=r_{t+1}+\gamma V^{\pi}\left(s^{\prime}\right)
\end{gathered}
$$

The TD method updates the state-value function using a prediction error called the TD error defined by

$$
\Delta=\left[r_{t+1}+\gamma V^{\pi}\left(s^{\prime}\right)\right]-V(s)
$$

The TD error $\Delta$ is the difference between the current and the old estimate of the statevalue function. The TD method then updates the state-value function as follows [28]

$$
V_{k+1}\left(s_{t}\right)=V_{k}\left(s_{t}\right)+\alpha\left[r_{t+1}+\gamma V_{k}\left(s_{t+1}\right)-V_{k}\left(s_{t}\right)\right]
$$

where $\alpha \in[0,1]$ is the learning rate, $\gamma$ is the discount factor and $r_{t+1}$ is the received immediate reward at time $t+1$. The TD learning algorithm is shown in Algorithm 2.

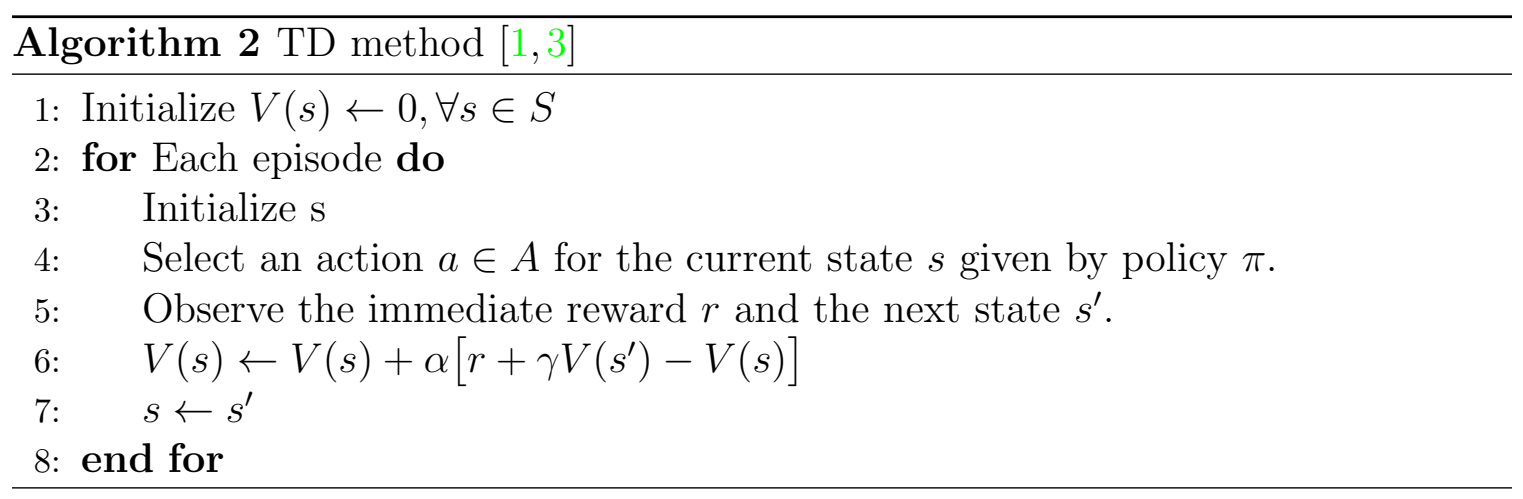




\subsubsection{Q-Learning}

The Q-learning algorithm, introduced by [13], is one of the most popular modelfree reinforcement learning methods. It enables agents to learn how to take optimal actions in Markovian domains by experiencing the consequences of actions [1]]. For learning problems in the discrete domain, the Q-learning method can estimate an optimal action-value function $Q^{*}(s, a)$ for all state-action pairs based on the TD error [9]. Problems in the continuous domain require the action and state space to be discretized in order to setup the Q-function. However, the number of state-action pairs in the Q-function becomes large when we perform a fine discretization leading to slow learning performance [9]. The Q-learning algorithm is similar to the TD learning algorithm. Like the TD learning algorithm, the Q-learning algorithm also computes the prediction or TD error similar to that of Equation 3.17. The $Q$-learning algorithm computes the prediction error as

$$
\Delta=\left[r_{t+1}+\gamma \max _{a} Q_{k}\left(s_{t+1}, a\right)\right]-Q_{k}\left(s_{t}, a_{t}\right)
$$

The state-action value is then updated as

$$
Q_{k+1}\left(s_{t}, a_{t}\right)=Q_{k}\left(s_{t}, a_{t}\right)+\alpha\left[r_{t+1}+\gamma \max _{a} Q_{k}\left(s_{t+1}, a\right)\right]-Q_{k}\left(s_{t}, a_{t}\right)
$$

The Q-learning algorithm can be written as Algorithm 3.

\subsubsection{Actor-Critic Learning}

Actor-critic learning methods are an extension of the TD learning methods [3]. In the actor-critic method the policy is kept separate and independent of the value function. The critic (state-value function) evaluates or criticizes the actions taken by the actor 




(the policy) [11]. The task of the critic is to predict the future system performance and the value of the state. After an action has been taken, the critic assesses the action to determine whether it was good or bad. The critic evaluates the action using the TD error defined previously by Equation 3.17. The goal of the TD error is to strengthen or weaken the selection of the action $a_{t}$ in state $s_{t}$. Figure 3.2 shows the actor-critic architecture. [11].

\subsection{Continuous State and Action Spaces}

Reinforcement learning (RL) methods are generally restricted to problems with small discrete state-action spaces $[3,4]$. In applications where the state-action spaces are very large or continuous, a function approximation method is required. In this thesis, the RL problems presented have continuous state-action spaces. Therefore, we use a fuzzy inference system (FIS) as a function approximator to represent the continuous state and action space. RL methods have been used in combination with FIS in previous research. Lionel Jouffe [6] introduced two methods that combine actor-critic 


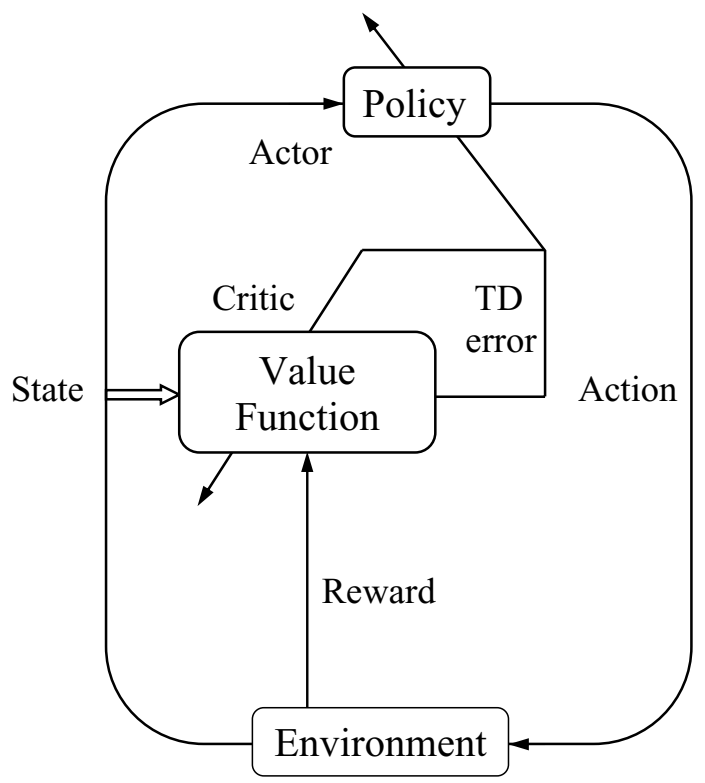

Figure 3.2: The actor-critic architecture

learning methods with FIS namely, fuzzy $Q$-Learning (FQL) and fuzzy actor-critic learning (FACL).

\subsubsection{Fuzzy Actor-Critic Learning}

Actor-critic learning methods consists of two parts: the actor which chooses the optimal action for each state and the critic which uses an approximation architecture to learn a value function of each state [30]. The fuzzy actor-critic learning (FACL) algorithm is based on principles where the actor and critic are modeled as fuzzy inference systems. The only available information for learning is the system's rewards, which is associated with the task the learning agent has to realize [6]. At each time step, the agent receives some representation of the environment's state $s_{t} \in S$, where $S$ is a continuous set of possible states as inputs to the system. The actor, based on the inputs, selects an optimal action $a_{t} \in A\left(s_{t}\right)$, where $A$ is a continuous set of actions available in $s_{t}$. The critic estimates the future system performance by evaluating the 
value function of the learning agent with respect to its goal. One time step later in part as a consequence of its action - the learning agent receives a reinforcement signal, $r_{t+1} \in R$ and finds itself in a new state $s_{t+1}$. The reinforcement signal, the value function of the current state, $\hat{V}_{t}$ and the next state, $\hat{V}_{t+1}$ are used to evaluate the Temporal Difference (TD) error (prediction error) which is used to adapt the inputs of both the actor and critic. Both actor and critic are implemented as a zero order Takagi-Sugeno (TS) fuzzy inference system (FIS) with constant consequents as described in Chapter 2. The output of the actor, $U_{t}$, is expressed as

$$
U_{t}=\sum_{l=1}^{L} \bar{\varphi}_{t}^{l} w_{t}^{l}
$$

where $L$ is the number of rules and $w^{l}$ is the conclusion parameter of the actor for rule $l$. The variable $\bar{\varphi}_{t}^{l}$ is the normalized firing strength for rule $l$ expressed as

$$
\bar{\varphi}_{t}^{l}=\frac{\prod_{j=1}^{J} \mu_{A_{j}^{l}}\left(x_{j}\right)}{\sum_{l=1}^{L}\left(\prod_{j=1}^{J} \mu_{A_{j}^{l}}\left(x_{j}\right)\right)}
$$

and can also be expressed in terms of the firing strength as

$$
\bar{\varphi}_{t}^{l}=\frac{\varphi_{t}^{l}}{\sum_{l=1}^{L} \varphi_{t}^{l}}
$$

where $\varphi_{t}^{l}$ is the firing strength defined as

$$
\varphi_{t}^{l}=\prod_{j=1}^{J} \mu_{A_{j}^{l}}\left(x_{j}\right)
$$


For adequate exploration of the action space, a random white noise $N_{t}(0, \sigma)$ is introduced into the control signal $U_{t}$ to produce a final noisy control $U_{t}^{\prime}$ as shown in figure 3.3. The critic estimates the state-value function $V_{t}$. The output of the critic $\hat{V}_{t}$ is expressed as

$$
\hat{V}_{t}=\sum_{l=1}^{L} \bar{\varphi}_{t}^{l} \zeta_{t}^{l}
$$

where $\zeta_{t}^{l}$ is the conclusion parameter of the critic for rule $l$. The external reward signal $r_{t+1}$ received from the environment after taking action $U_{t}$ in state $s_{t}$ and transitioning into the next state $s_{t+1}$ is used to compute the temporal difference $(T D)$ error $\Delta$ defined as

$$
\Delta=r_{t+1}+\gamma \hat{V}_{t+1}-\hat{V}_{t}
$$

where $\gamma \in[0,1]$ is the discount factor. The TD error $\Delta$ is used to update both the actor and the critic. We update the output parameter of the actor $w^{l}$ as

$$
w_{t+1}^{l}=w_{t}^{l}+\beta \Delta N_{t} \frac{\partial U_{t}}{\partial w_{t}^{l}}
$$

where $\beta \in(0,1)$ is the actor's learning rate, $N_{t}$ is the noise and the partial derivative in Equation 3.27 can be derived from Equation 3.21 as

$$
\frac{\partial U_{t}}{\partial w_{t}^{l}}=\frac{\prod_{i=1}^{n} \mu_{A_{i}{ }^{l}}\left(x_{i}\right)}{\sum_{l=1}^{L}\left(\prod_{i=1}^{n} \mu_{A_{i}{ }^{l}}\left(x_{i}\right)\right)}=\varphi_{t}^{l}
$$

The update law for the output parameter of the critic $\zeta^{l}$ is expressed as

$$
\zeta_{t+1}^{l}=\zeta_{t}^{l}+\alpha \Delta \frac{\partial \hat{V}_{t}}{\partial \zeta^{l}}
$$


Where $\alpha \in(0,1)$ is the learning rate for the critic. The partial derivative in Equation 3.29 can be derived from Equation 3.25 as

$$
\frac{\partial \hat{V}_{t}}{\partial \zeta_{t}^{l}}=\frac{\prod_{i=1}^{n} \mu_{A_{i}}^{l}\left(x_{i}\right)}{\sum_{l=1}^{L} \prod_{i=1}^{n} \mu_{A_{i}}^{l}\left(x_{i}\right)}=\varphi_{t}^{l}
$$

We set $\alpha>\beta$ so that the critic will converge more quickly than the actor [28]. The summary of the FACL algorithm is shown in algorithm 4 [28]

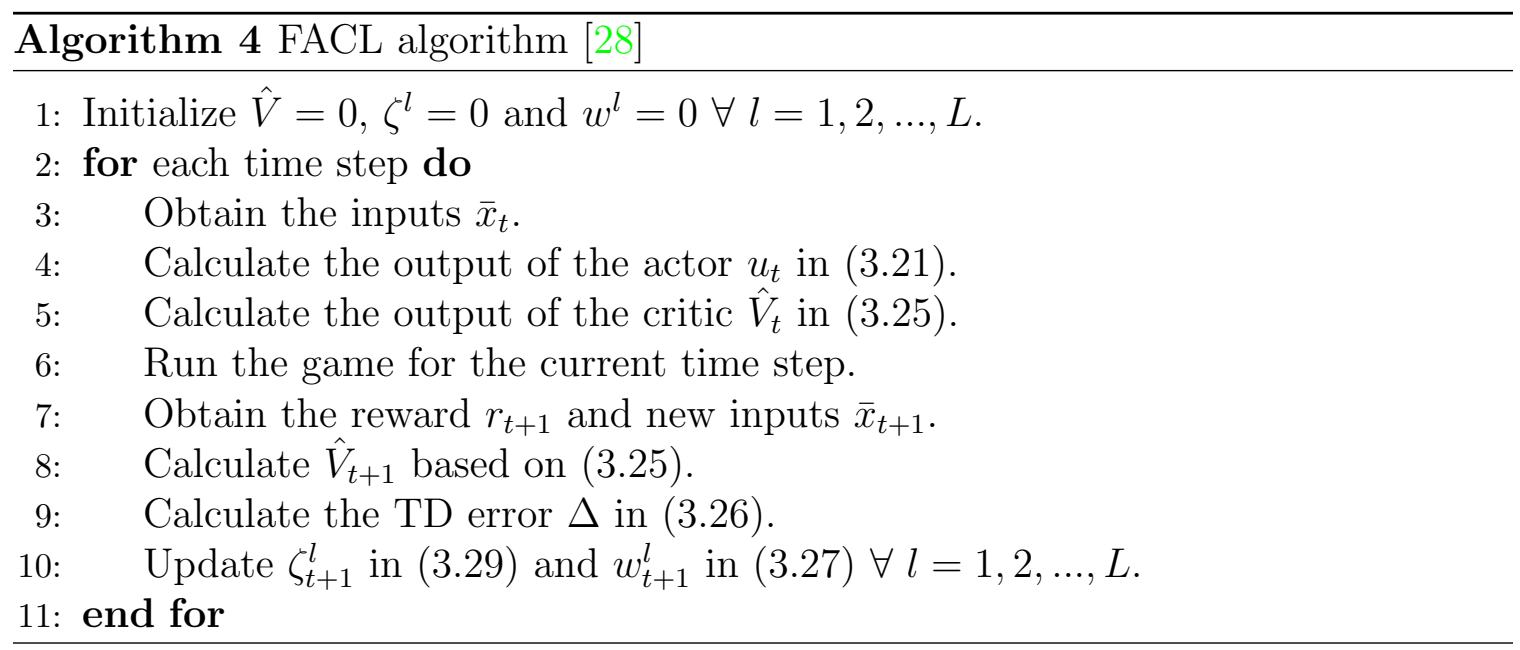

\subsection{2 $\mathrm{Q}(\lambda)$-Learning Fuzzy Inference System}

The $\mathrm{Q}(\lambda)$-learning fuzzy inference system (QLFIS) enables the use of Q-learning in problems with continuous or very large discrete state and action spaces. The QLFIS uses two fuzzy inference system (FIS) just like in the FACL. In order to differentiate the two FIS, one is refered to as a fuzzy logic controller (FLC) and the other is refered to as the FIS. The FLC and FIS are implemented using zero-order Takagi-Sugeno (TS) fuzzy inference with constant conclusions. The FIS estimates the optimal action-value function $Q^{*}(s, a)$ and the FLC determines the control action. $Q(\lambda)$-learning is used to tune the parameters of both FLC and FIS [1, 1$]$. 




Figure 3.3: The fuzzy actor-critic learning (FACL) control structure

The action-value function $Q\left(s_{t}, a_{t}\right)$, for a given state $s_{t}$ and action $a_{t}$ is updated as

$$
Q_{t+1}\left(s_{t}, a_{t}\right)=Q_{t}\left(s_{t}, a_{t}\right)+\eta \Delta_{t} e_{t}
$$

where $(0<\eta \leq 1)$ is the learning rate, $e_{t}$ is the eligibility trace and $\Delta_{t}$ is the temporal difference error defined as

$$
\Delta_{t}=r_{t+1}+\gamma \max _{a} Q_{t}\left(s_{t+1}, \dot{a}\right)-Q_{t}\left(s_{t}, a_{t}\right)
$$

The eligibility trace $e_{t}$ is thought to speed up the learning process and is defined as $[1,4]$

$$
e_{t}=\gamma \lambda e_{t-1}+\frac{\partial Q_{t}\left(s_{t}, a_{t}\right)}{\partial \Gamma}
$$

where $\lambda$ is the trace-decay parameter, $(0 \leq \lambda \leq 1)$ and $\Gamma$ is the parameter to be tuned. The study presented in [1] showed that using eligibility traces did not lead to 
significant improvement of learning speed when compared to the QLFIS algorithm without using eligibility traces. Therefore, we remove the eligibility trace by setting $\lambda=0$. Therefore, the variable $e_{t}$ in (3.31) and (3.33) becomes

$$
e_{t}=\frac{\partial Q_{t}\left(s_{t}, a_{t}\right)}{\partial \Gamma}
$$

The output of the FIS is the estimate of the action-value function $\hat{Q}\left(s_{t}, u_{t}\right)$ defined as

$$
\hat{Q}_{t}\left(s_{t}, a_{t}\right)=\sum_{l=1}^{L} \bar{\varphi}_{t}^{l} q_{t}^{l}
$$

where $q_{t}^{l}$ is the output conclusion parameter of the FIS to be tuned and $\bar{\varphi}$ is the normalized firing strength defined in Equation 3.22. The output of the FLC $a_{t}$ is defined as

$$
a_{t}=\sum_{l=1}^{L} \bar{\varphi}_{t}^{l} b_{t}^{l}
$$

where $b_{t}^{l}$ is a set of possible actions and the output conclusion parameter of the FLC to be tuned. Assume the input space(s) of the FLC and FIS are covered using Gaussian membership functions which are defined by

$$
\mu_{A_{j}^{l}}\left(x_{j}\right)=\exp \left(-\left(\frac{x_{j}-c_{j}^{l}}{\sigma_{j}^{l}}\right)^{2}\right) \forall j \in J, \forall l \in L
$$

where $J$ is the number of inputs and $L$ is the number of rules. The mean $c$ and the standard deviation $\sigma$, are the input parameters to be tuned. We define the vector of 
the input and output parameters of both FIS and FLC as

$$
\Gamma_{F I S, F L C}=\left(\begin{array}{c}
q_{t}^{l}, b_{t}^{l} \\
c_{j}^{l} \\
\sigma_{j}^{l}
\end{array}\right)
$$

The update law for the FIS parameters is defined as [4]

$$
\Gamma_{F I S}(t+1)=\Gamma_{F I S}(t)+\alpha \Delta_{t} \frac{\partial \hat{Q}_{t}\left(s_{t}, u_{t}\right)}{\partial \Gamma_{F I S}}
$$

and the update law for the FLC parameters is defined as

$$
\Gamma_{F L C}(t+1)=\Gamma_{F L C}(t)+\beta \Delta_{t} \frac{\partial u}{\partial \Gamma_{F L C}} N_{t}
$$

where $N_{t}\left(0, \sigma_{n}\right)$ is the random noise, $\alpha$ is the learning rate for the FIS, $\beta$ is the learning rate for the FLC,

$$
\frac{\partial \hat{Q}_{t}\left(s_{t}, a_{t}\right)}{\partial \Gamma_{F I S}}=\left(\begin{array}{c}
\frac{\partial \hat{Q}_{t}\left(s_{t}, a_{t}\right)}{\partial q_{t}^{l}} \\
\frac{\partial \hat{Q}_{t}\left(s_{t}, a_{t}\right)}{\partial c_{j}^{l}} \\
\frac{\partial \hat{Q}_{t}\left(s_{t}, a_{t}\right)}{\partial \sigma_{j}^{l}}
\end{array}\right)=\left(\begin{array}{c}
\bar{\varphi}_{l} \\
\frac{\left(q_{t}^{l}-\hat{Q}_{t}\left(s_{t}, a_{t}\right)\right)}{\sum_{l} \varphi_{l}} \varphi_{l} \frac{2\left(x_{j}-c_{j}^{l}\right)}{\left(\sigma_{j}^{l}\right)^{2}} \\
\frac{\left(q_{t}^{l}-\hat{Q}_{t}\left(s_{t}, a_{t}\right)\right)}{\sum_{l} \varphi_{l}} \varphi_{l} \frac{2\left(x_{j}-c_{j}^{l}\right)^{2}}{\left(\sigma_{j}^{l}\right)^{3}}
\end{array}\right)
$$

and

$$
\frac{\partial a}{\partial \Gamma_{F L C}}=\left(\begin{array}{c}
\frac{\partial a}{\partial b_{t}^{l}} \\
\frac{\partial a}{\partial c_{j}^{l}} \\
\frac{\partial a}{\partial \sigma_{j}^{l}}
\end{array}\right)=\left(\begin{array}{c}
\bar{\varphi}_{l} \\
\frac{\left(b_{t}^{l}-a\right)}{\sum_{l} \varphi_{l}} \varphi_{l} \frac{2\left(x_{j}-c_{j}^{l}\right)}{\left(\sigma_{j}^{l}\right)^{2}} \\
\frac{\left(b_{t}^{l}-a\right)}{\sum_{l} \varphi_{l}} \varphi_{l} \frac{2\left(x_{j}-c_{j}^{l}\right)^{2}}{\left(\sigma_{j}^{l}\right)^{3}}
\end{array}\right)
$$


The QLFIS algorithm is summarized in Algorithm 5



\subsection{Discussion}

In this chapter, we studied the concept of the reinforcement learning problem and its elements. These elements include the reinforcement learning agent, its environment, the reward and the agent's policy. We reviewed Markov decision processes and the Markov property. We discussed temporal-difference learning methods, Q-learning and actor-critic learning methods. We discussed the $\mathrm{Q}(\lambda)$-learning fuzzy inference system (QLFIS) method and the fuzzy actor-critic learning methods. The QLFIS algorithm was successfully used to train the players in pursuit-evasion differential (in Chapter 4) games using Gaussian membership functions in $[1,4]$. We propose that the FACL algorithm using only triangular membership functions and updating only the 
output parameters of the fuzzy system will work as well as (or better than) the QLFIS algorithm in $[1,4]$. The QLFIS method in $[1,4]$ requires six parameters to be updated for every fuzzy rule from (3.41) and (3.42). There are also more computations to be done in updating the two input parameters. In addition, when using Gaussian membership functions, there is a possibility that all the rules fire at the same time for some inputs. In our approach, we update only the output parameters in (3.27) and (3.29). We construct the triangular membership function in a way that only two membership functions can be activated per input and there is no possibility for all rules to fire at the same time. The simulations and results of our approach is discussed in Chapter 5. 


\section{Chapter 4}

\section{Differential Games}

\subsection{Pursuit-evasion Differential Game}

Differential games (DG), introduced by Rufus Isaccs [2], are a category of dynamic, continuous time games. They offer a theoretical model for studying the interactions between multiple players in systems which are represented by differential equations. The players usually have conflicting goals (zero-sum games). Some differential games include the guarding the territory game, the pursuit-evasion game and many other problems. In this thesis we will focus on pursuit-evasion games. In pursuit-evasion games, one or a number of pursuers attempt to capture one or a number of evaders in minimal time while the evader(s) try to avoid or delay capture. The difficulty of the problem is due to the conflicting goals of the players who try to make the best decisions considering their opponents are trying to do the same [2].

Two of the most familiar pursuit-evasion games are the; homicidal chauffeur game, and the game of two cars. In our version of both games, each game terminates when the evader is within the lethal range of the pursuer(capture), or when the time exceeds one minute (escape). The players evaluate their current state and then select their next actions. Each player has no knowledge of the other player's next selected 
action. We assume that the environment is obstacle free. Conventional reinforcement learning algorithms like Q-learning may prove computationally cumbersome because of the continuous state and action space [28]. We use fuzzy systems to represent the continuous state and action space in order to avoid this problem.

The Isaacs condition given in [31] determines if an optimal strategy exists in the homicidal chauffeur differential game. The homicidal chauffeur game is discussed in subsection 5.2.1 as well as the game of two car in subsection 4.1.2 .

\subsubsection{The Homicidal Chauffeur Problem [1,2]}

The homicidal chauffeur problem is presented as one example of a pursuit-evasion differential game. In this game, a pursuer (chauffeur) aims to minimize the capture time of an evader(pedestrian). The evader's objective is to maximize the capture time and, if possible, avoid capture. The chauffeur is a car-like mobile robot and is denoted as $P$. The pedestrian, denoted as $E$, is a point that can move instantaneously in any direction.

We assume that the players move at a constant forward speed $v_{i}, i \in(p, e)$. The pursuer's speed is greater than the evader's speed, but the evader can move in any direction instantaneously. We constrain the motion of the pursuer by its maximum steering angle such that

$$
-\left(u_{p \max }\right) \leq u_{p} \leq\left(u_{\max }\right)
$$

where $\left(u_{\text {pmax }}\right)$ is the maximum steering angle.

The maximum steering angle results in a minimum turning radius $R_{p}$ defined by

$$
R_{p}=\frac{L_{p}}{\tan \left(u_{p \max }\right)}
$$

where $L_{p}$ is the pursuer's wheelbase. 


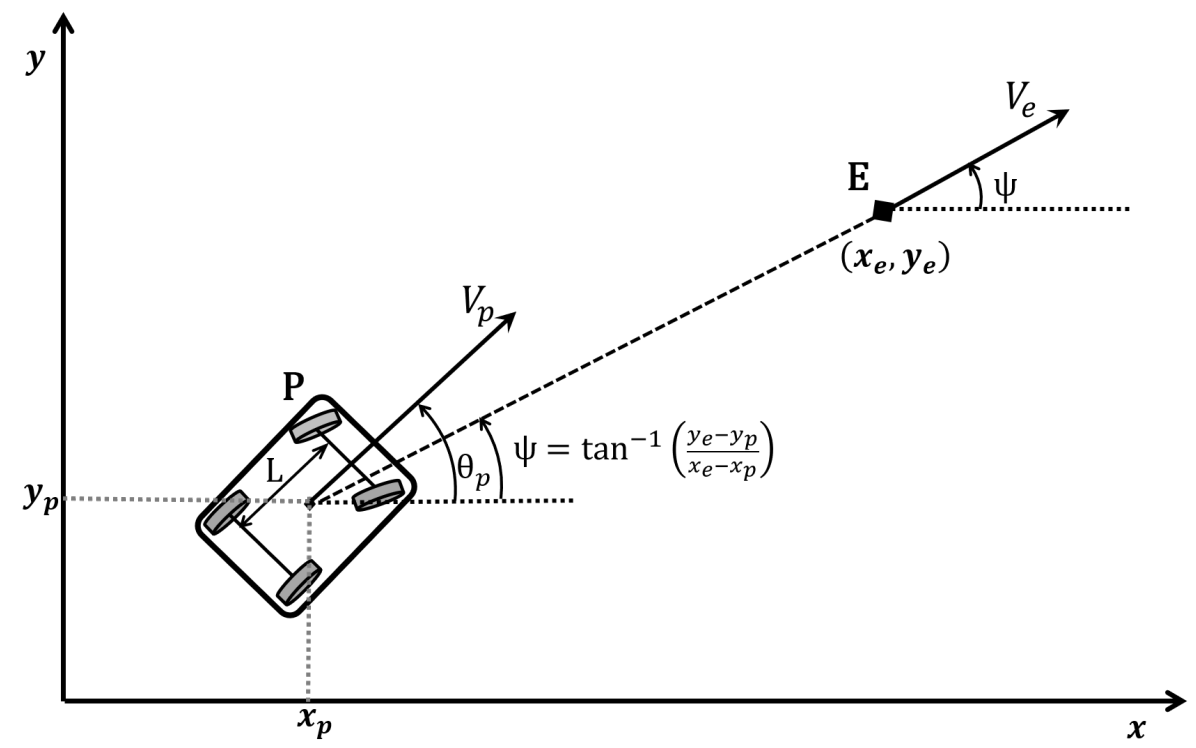

Figure 4.1: Homicidal chauffeur problem [1]

The kinematic equations for the pursuer $\mathrm{P}$ and the evader E are [2]

$$
\begin{aligned}
& \dot{x}_{p}=v_{p} \cos \left(\theta_{p}\right) \\
& \dot{y}_{p}=v_{p} \sin \left(\theta_{p}\right) \\
& \dot{\theta}_{p}=\frac{v_{p}}{L_{p}} \tan \left(u_{p}\right) \\
& \dot{x}_{e}=v_{e} \cos \left(u_{e}\right) \\
& \dot{y}_{e}=v_{e} \sin \left(u_{e}\right)
\end{aligned}
$$

where $(x, y), v$, and $\theta$ denote the position, the velocity, and the orientation respectively as shown in Fig. 4.1.

The line of sight $\psi$ from the pursuer to the evader is given as

$$
\psi=\tan ^{-1}\left(\frac{y_{e}-y_{p}}{x_{e}-x_{p}}\right)
$$


The angle difference $\phi$ between the pursuer and the evader is given as

$$
\phi=\left(\psi-\theta_{p}\right)
$$

The relative distance between pursuer and evader is found as

$$
d=\sqrt{\left(x_{e}-x_{p}\right)^{2}+\left(y_{e}-y_{p}\right)^{2}}
$$

The capture occurs when the distance $d \leq \ell$ where $\ell$ is capture radius.

According to [2], there is capture condition in order for the pursuer to be able to capture the evader given as

$$
\ell / R_{p}>\sqrt{1-\gamma^{2}}+\sin ^{-1} \gamma-1
$$

where $\ell / R_{p}$ is the ratio of the radius of capture to the minimum turning radius of the pursuer, and $\gamma=v_{e} / v_{p}<1$ is the ratio of the evader's speed to the pursuer's speed. Assuming that the pursuer's speed is greater than the evader's speed. If the inequality in (4.7) is not satisfied, E escapes from P indefinitely.

The pursuer's optimal control strategy is to minimize the distance between itself and the evader and capture the evader in minimum time. The pursuer controls its steering angle as follows $[4,17,31]$

$$
u_{p}=\tan ^{-1}\left(\frac{y_{e}-y_{p}}{x_{e}-x_{p}}\right)-\theta_{p}
$$

The evader's optimal strategies are given as $[2,18]$ :

1- When the pursuer is far enough from the evader, maximize the distance from the current position of the pursuer by running away 
from the pursuer and along the pursuer's line-of-sight as follows

$$
u_{e}=\tan ^{-1} \frac{y_{e}-y_{p}}{x_{e}-x_{p}}
$$

2- When the pursuer is close to the evader such that $d \leq R_{p}$, the evader will make a sharp turn normal to its direction and enter the pursuer's non-holonomic constraint region. The pursuer cannot turn more than its minimum turning radius $R_{p}$. For the evader to use the advantage of its higher maneuverability, the second control strategy is given as

$$
u_{e_{\text {extreme }}}=\theta_{e} \pm \pi / 2
$$

Figure 4.2 shows that a non-holonomic wheeled player is constrained to move along the path with a bounded curvature denoted as two dotted circles which is the pursuer's minimum turning radius $R$ given in (4.2).

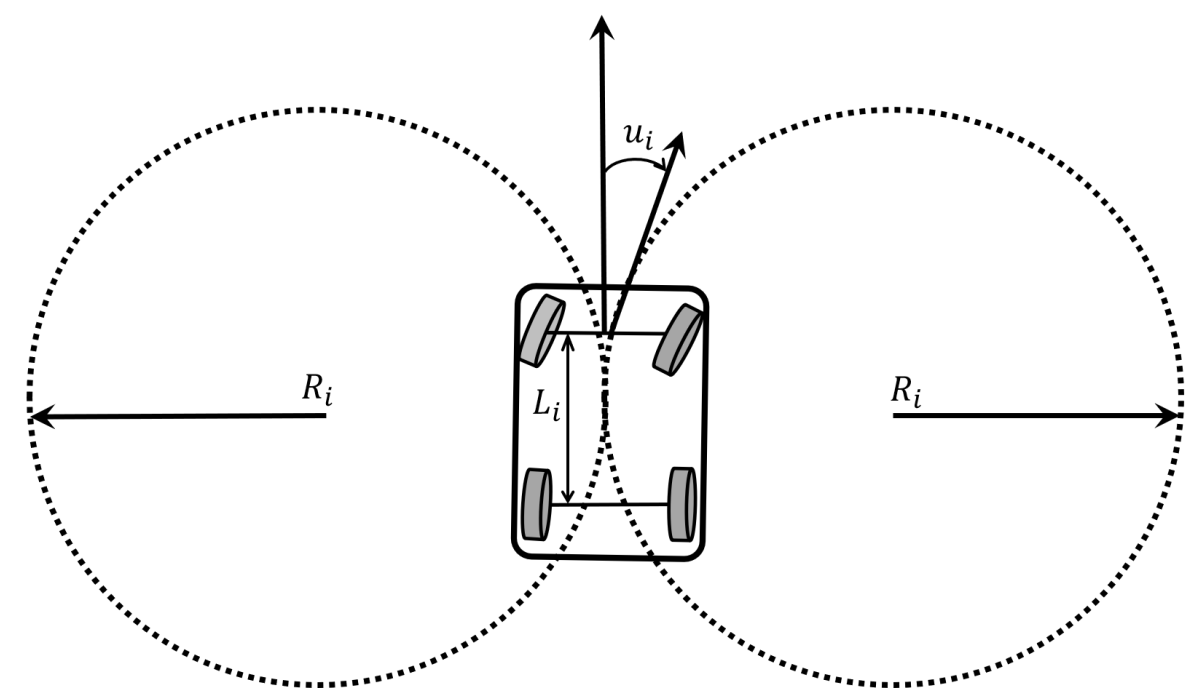

Figure 4.2: The vehicle cannot turn into the circular region defined by its minimum turning radius $R[1]$ 


\subsubsection{The Game of Two Cars $[1,2]$}

Unlike the homicidal chauffeur problem, the game of two cars involves two car-like mobile robots. The evader in this game is modeled like a car and not like a pedestrian that can change its direction instantaneously. The pursuer is faster than the evader but the evader is more maneuverable than the pursuer such that

$$
\begin{aligned}
& v_{p}>v_{e} \\
& u_{p_{\max }}<u_{e_{\max }}
\end{aligned}
$$

The kinematic equations for the pursuer and the evader are $[2,16]$ :

$$
\begin{aligned}
& \dot{x}_{i}=v_{i} \cos \left(\theta_{i}\right) \\
& \dot{y}_{i}=v_{i} \sin \left(\theta_{i}\right) \\
& \dot{\theta}_{i}=\frac{v_{i}}{L_{i}} \tan \left(u_{i}\right)
\end{aligned}
$$

where $i \in(p, e)$, and $\left(x_{i}, y_{i}\right), v_{i}, \theta_{i}$, and $L_{i}$ denote the position, the velocity, the orientation, and the wheelbase of the robot respectively. The parameters and the model are shown in Figure 4.3.

The angle difference $\phi_{i}$ between the pursuer and the evader is given as

$$
\phi_{i}=\tan ^{-1}\left(\frac{y_{e}-y_{p}}{x_{e}-x_{p}}\right)-\theta_{i}
$$

Unlike the homicidal chauffeur game, there is no capture condition for the game of two cars. However, there is a time-optimal strategy proposed by [17]. The pursuer's strategy is to minimize the distance and the time of capture by controlling its steering angle $u_{p}$ by (4.8). 


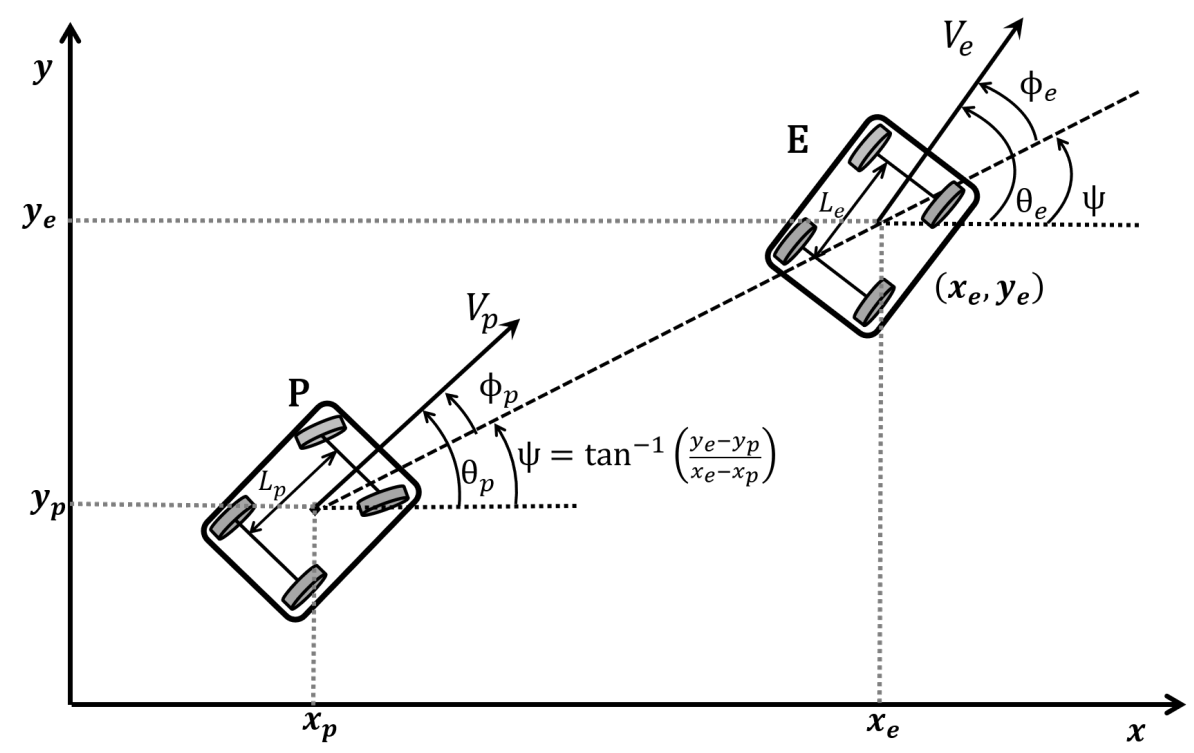

Figure 4.3: The game of two cars [1]

The evader uses two different control strategies:

1- When the pursuer is far from the evader, the evader's control strategy is to maximize the distance by running away along the pursuer's line-of-sight and controlling its steering angle as

$$
u_{e}=\tan ^{-1}\left(\frac{y_{e}-y_{p}}{x_{e}-x_{p}}\right)-\theta_{e}
$$

2- When the distance between the pursuer and the evader is less than $R_{p}$, the evader's second control strategy is to make a sharp turn $u_{e_{\max }}$ to enter the pursuer's non-holonomic constraint region. This is given as

$$
u_{e}=\left(\theta_{p}+\pi\right)-\theta_{e}
$$

In [17], the pursuer was trained to capture the evader while the evader plays its optimal strategy. In [1], the pursuer and the evader are simultaneously trained 
using the $Q(\lambda)$-learning fuzzy inference system (QLFIS) discussed in Chapter 3. In this thesis, we apply the fuzzy actor-critic learning (FACL) algorithm to train both pursuer and evader. We focus mainly on the evader learning to take sharp turns and escape using its second strategy in (4.10) for the homicidal chauffeur game and (4.15) for the game of two cars. Gaussian membership functions were constructed over the input state spaces in [1], and input and output parameters were tuned. We believe that the evader can learn just as well or even better when its input spaces are covered with only triangular membership functions and only output parameters are tuned. We believe our approach will save some computation time when compared with the approach in [1]. We will present the results of our approach in Chapter 5 and compare to the results obtained when we use the method in [1] .

\subsection{Homing Missile Guidance}

In the homicidal chauffeur game and the game of two cars, the input is a steering angle. In this example of a homing missile guidance problem, the input is a force and the missile is treated as an inertial element. We limit the input to only applying a normal force such that speed does not change but the direction of the velocity vector changes. A missile system that is equipped with homing guidance is able to sense the target and steer itself towards the target by commanding its own control surfaces [32].

One of the most widely used homing guidance technique for short to medium range missiles is the proportional navigation (PN) guidance. A classical PN approach stipulates that two bodies closing in on each other will eventually collide when the line of sight(LOS) rate between the two bodies is zero [32,33]. Unlike the previous games mentioned in the this chapter, the missile does not follow the target directly along its LOS but takes a different path to collision. The PN guidance technique 
ensures the efficient interception of a non maneuvering target. Both missile and target are modeled as a point mass and the missile-target engagement geometry is illustrated in Figure 4.4, where the subscript $M$ and $T$ denotes the missile and the target respectively. To intercept the target, the missile must keep a constant angle

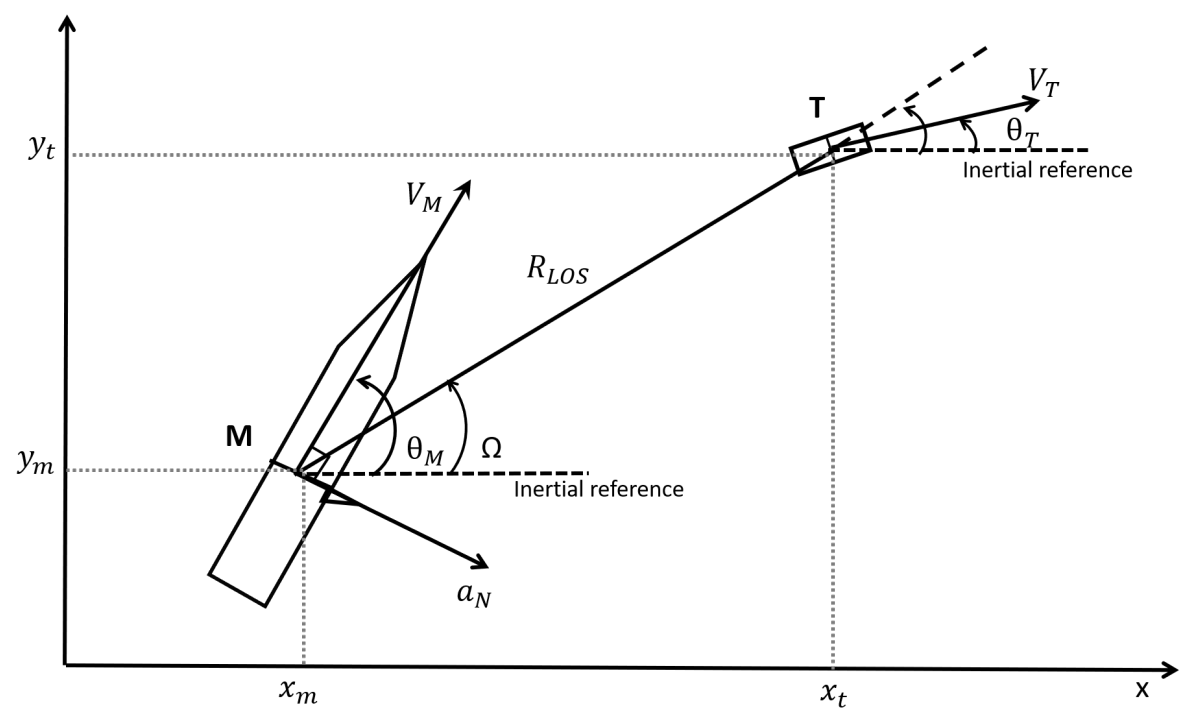

Figure 4.4: Missile-Target Geometry

with the LOS to the target. If the missile flight path changes at the same rate as the LOS, the missile will have to change its direction at an increasing rate and may not intercept the target. In order to achieve the desired collision course, the missile's flight path rotation must be greater than the LOS rotation [34]. The ratio of the missile's flight path rotation to the LOS rotation is called the navigation ratio $N$. The PN law is given as

$$
\dot{\theta}_{M}=N \dot{\Omega}
$$

where $\theta_{M}$ and $\Omega$ are the missile's flight path angle and the LOS angle, $\dot{\theta}_{M}$ and $\dot{\Omega}$ are the angular rate of the missile's velocity vector and the angular rate of the LOS, and $N>1$ is the navigation ratio, respectively. 
There are many variations of the PN guidance law and the difference lies in how the commanded acceleration is applied [35]. In this study, the acceleration command will be applied in the direction normal to the missile's velocity vector $V_{M}$ which does not change the missile speed, but leads to a rotation of the velocity vector. To derive the equation for the normal acceleration, we apply the principle of uniform circular motion in Figure 4.5. For a straight moving object to navigate a curved path, an external force that is normal to the object's forward velocity is required. This normal force changes the direction of the object without changing its speed. The resulting normal acceleration will be in the direction of the force and pointed towards the center of the curved path. We apply this principle to the missile in Figure 4.4. The equation

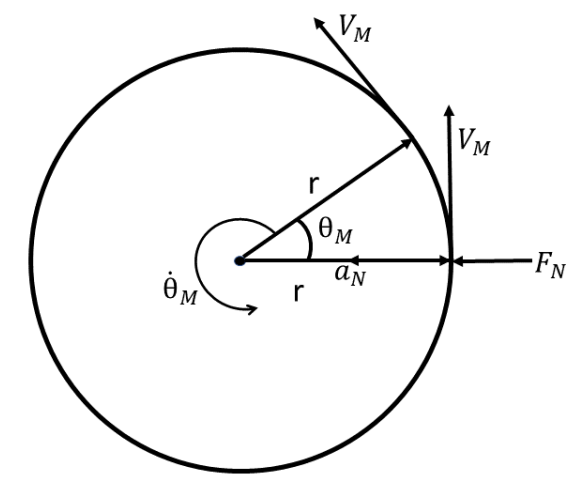

Figure 4.5: Missile-Target Geometry

for the normal force according to Newton's second law is given as

$$
F_{N}=m a_{N}
$$

where $m$ is the mass of the missile and $a_{N}$ is the normal acceleration. The normal 
acceleration is defined as

$$
a_{N}=\frac{V_{M}^{2}}{r}
$$

where $r$ is the radius of the circle that best fits the curved path. The angular velocity $\dot{\theta}_{m}$ for circular motion is defined as

$$
\dot{\theta}_{M}=\frac{V_{M}}{r}
$$

Substituting the right-hand-side of (4.19) into (4.18), the normal acceleration becomes

$$
a_{N}=V_{M} \dot{\theta}_{M}
$$

We recall that the guidance law requires that $\theta_{M}$ be proportional to the rate of change of the LOS angle $\dot{\Omega}$ from (4.16). The commanded acceleration is then written as

$$
a_{N}=N V_{M} \dot{\Omega}
$$

In order to compute the commanded normal acceleration given in $(4.21)$, we need to measure the angular rate of rotation $\dot{\Omega}$. In practice, $\dot{\Omega}$ is usually measured by a seeker located at the nose of the missile. We first define some useful equations in order to derive $\dot{\Omega}$.

The vector defining the line of sight (LOS) from the missile to the target is

$$
R_{\text {Los }}=\left[\begin{array}{c}
x_{\text {Los }} \\
y_{\text {Los }}
\end{array}\right]=\left[\begin{array}{c}
x_{t}-x_{m} \\
y_{t}-y_{m}
\end{array}\right]
$$


The vector defining relative velocity of the missile relative to the target is

$$
V_{r}=V_{m}-V_{t}=\left[\begin{array}{c}
\dot{x}_{m}-\dot{x}_{t} \\
\dot{y}_{m}-\dot{y}_{t}
\end{array}\right]
$$

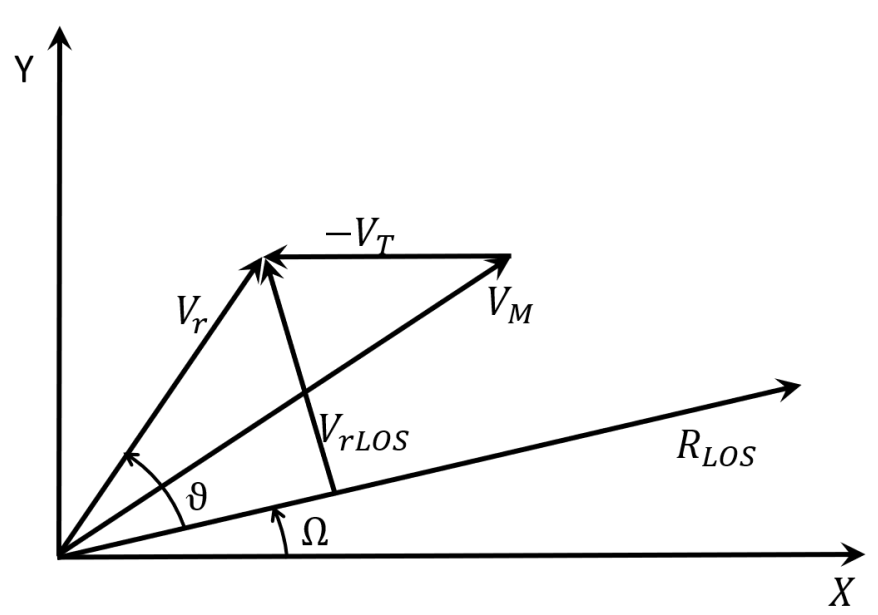

Figure 4.6: Components for navigation

The angle between the LOS vector $R_{\text {Los }}$ and the relative velocity vector $V_{r}$ is denoted by $\vartheta$ in Figure 4.6 which is defined as

$$
\vartheta=\angle R_{L} o s-\angle V_{r}=\tan ^{-1}\left(\frac{y_{L o s}}{x_{L o s}}\right)-\tan ^{-1}\left(\frac{\dot{y}_{m}-\dot{y}_{t}}{\dot{x}_{m}-\dot{x}_{m}}\right)
$$

The component of relative velocity perpendicular to the LOS $V_{r L o s}$ from is

$$
V_{r L o s}=\left|V_{r}\right| \sin \vartheta
$$

The angular rate of rotation of the LOS is then written as

$$
\dot{\Omega}=\frac{V_{r L o s}}{\left|R_{L o s}\right|}
$$


Substituting (4.26) into (4.21), we get the acceleration command

$$
\left|a_{N}\right|=\frac{N\left|V_{m}\right| V_{r L o s}}{\left|R_{\text {Los }}\right|}
$$

where $N \in[1,5]$. To resolve $a_{N}$ into its Cartesian coordinates, we first derive the unit normal to the missile's velocity vector. The unit normal is derived by rotating the unit vector parallel to the missile's velocity vector by $90^{\circ}$

$$
\begin{gathered}
\hat{n}_{v m}=\left[\begin{array}{c}
-\dot{y}_{m} \\
\left|V_{M}\right| \\
\dot{x}_{m} \\
\left|V_{M}\right|
\end{array}\right] \\
a_{N}=\left|a_{N}\right| \hat{n}_{v m}=\left[\begin{array}{c}
\ddot{x}_{m} \\
\ddot{y}_{m}
\end{array}\right]=\left[\begin{array}{c}
\frac{-\left|a_{N}\right| \dot{y}_{m}}{\left|V_{M}\right|} \\
\frac{\left|a_{N}\right| \dot{x}_{m}}{\left|V_{M}\right|}
\end{array}\right]
\end{gathered}
$$

To get the missile velocity, equation 4.29 is integrated once to get the missile velocity and integrated twice to get the missile position.

\subsection{Summary}

In this chapter, we discussed pursuit-evasion differential games. We discussed the players strategies in the homicidal chauffeur game and the game of two cars. We introduced the inertial homing missile guidance problem and the proportional navigation law which is popularly used in missile guidance problems. 


\section{Chapter 5}

\section{Fuzzy Actor-Critic Learning in Pursuit Evasion Games}

\subsection{Introduction}

In [4], the $\mathrm{Q}(\lambda)$-learning fuzzy inference system (QLFIS) algorithm was used to train the pursuer to capture the evader in pursuit-evasion differential games. The QLFIS algorithm, given in Algorithm 5, uses $\mathrm{Q}(\lambda)$-learning to tune the parameters of the fuzzy inference system (FIS). In [1], the QLFIS algorithm was used to train both the pursuer and the evader to learn their control strategies in pursuit-evasion differential games. Simulation results in [1] showed that using the QLFIS algorithm with eligibility traces does not significantly improve the learning speed of the evader. In both studies $[1,4]$, both input and output parameters of the FIS were tuned using the QLFIS learning algorithm.

In this thesis, we use the FACL algorithm to train both pursuer and evader to learn their control strategies. We study the evader's performance in two differential games; the homicidal chauffeur game and the game of two cars. The variable to be controlled is the evader's steering angle. Our main focus is the evader learning it's 
control strategy, especially learning to take sharp turns. We run simulations and compare the evader's performance with the results obtained when using the QLFIS algorithm described in $[1,4]$.

In addition, we use FACL to train an inertial homing missile to home in on its target. In the inertial homing missile guidance problem, the variable to be controlled is a normal force. Triangular membership functions are created and evenly spread over the input spaces of both the pursuer and evader in all games. We only tune, for each fuzzy rule, the output parameters of the pursuer and the evader in all games. This is in contrast to the QLFIS algorithm used in [1,4] where Gaussian membership functions are used, and both input and output parameters are tuned for each fuzzy rule. In comparison to the QLFIS algorithm in [1], we investigate the following;

- Whether using only triangular membership functions can successfully train the evader.

- How many membership functions it takes for the evader to learn.

- How increasing the number of triangular membership functions would affect computation time.

- Whether the evader can learn when we use inputs that were not considered in [1] such as; the line-of sight (LOS) from the pursuer to the evader $(\psi)$, and the angle difference between the pursuer's orientation $\left(\theta_{p}\right)$ and the evader orientation $\left(\theta_{e}\right)$.

\subsection{Simulation Results}

Please note that all simulations in this thesis were implemented using MATLAB programming software on a windows computer with a core i5 processor and 4GB RAM. We use fuzzy actor-critic learning (FACL) to train the evader to escape in 
the homicidal chauffeur game and the game of two cars. Although both pursuer and evader are learning to play the game simultaneously, the evader is our main focus. The pursuer and evader will be trained using the FACL algorithm in Algorithm 6 . We use triangular membership functions to cover the input space of both pursuer and evader. We compare the performance of the evader when we use different numbers of triangular membership functions. Our main goal is to train the evader to learn its second control strategy. The evader's second control strategy is to take sharp turns when the pursuer is within a certain distance described in Chapter 4. The triangular membership functions are defined as

$$
\mu_{A}\left(x_{i}\right)= \begin{cases}0, & x \leq a \\ \frac{x-a}{b-a}, & a \leq x \leq b \\ \frac{c-x}{c-b}, & b \leq x \leq c \\ 0, & c \leq x\end{cases}
$$

where $a, b$ and $c$ are the lower limit, peak and upper limit of the triangular membership function respectively.

The aim of the evader is to use the reward it receives at each time step to learn the appropriate actions. The pursuer aims to capture the evader in minimum time and the evader aims to delay capture or completely evade capture for the entire duration of the game. We use the distance between the pursuer and evader to create a reward function. The pursuer wants to minimize the distance while the evader wants to maximize the distance. The distance $D(t)$ at each time step $t$ is calculated by

$$
D(t)=\sqrt{\left(x_{e}(t)-x_{p}(t)\right)^{2}+\left(y_{e}(t)-y_{p}(t)\right)^{2}}
$$


and the change in distance $\Delta D(t)$

$$
\Delta D(t)=D(t)-D(t+1)
$$

The maximum change in distance is defined as $\Delta D_{\max }=V_{\text {rmax }} T$ where $V_{\text {rmax }}$ is the maximum relative velocity of the pursuer and the evader $\left(V_{\text {rmax }}=V_{p}+V_{e}\right)$ and $T$ is the sampling time. The evader receives a positive reward as it increases the distance between itself and the pursuer and a negative reward otherwise. We establish an immediate reward function similar to that of $[1,4]$. The immediate reward $R_{t+1}$ is given by

$$
R_{t+1}= \begin{cases}\frac{\Delta D(t)}{\Delta D_{\max }} & \text { for pursuer } \\ \frac{-\Delta D(t)}{\Delta D_{\max }} & \text { for evader }\end{cases}
$$

\subsubsection{Homicidal Chauffeur Game}

In our simulation of the homicidal chauffeur game, a single pursuer chases a single evader. In order for the evader to escape the pursuer, the evader must take sharp turns into the minimum radius constraint of the pursuer as described in Section 4.1. In $[1,4]$, three Gaussian membership functions were used in the QLFIS implementation. Both the input and the output fuzzy parameters are updated in [1,4], therefore 6 parameters are updated on each iteration. In our approach, we start by using three triangular membership functions in our FACL algorithm but we only update the output parameter of the FIS. However, we know that we can improve function approximation by using more membership functions as illustrated with the example in Subsection 2.2.3. We can therefore increase the number of membership functions over the inputs and observe the results. We measure the performance of the evader 


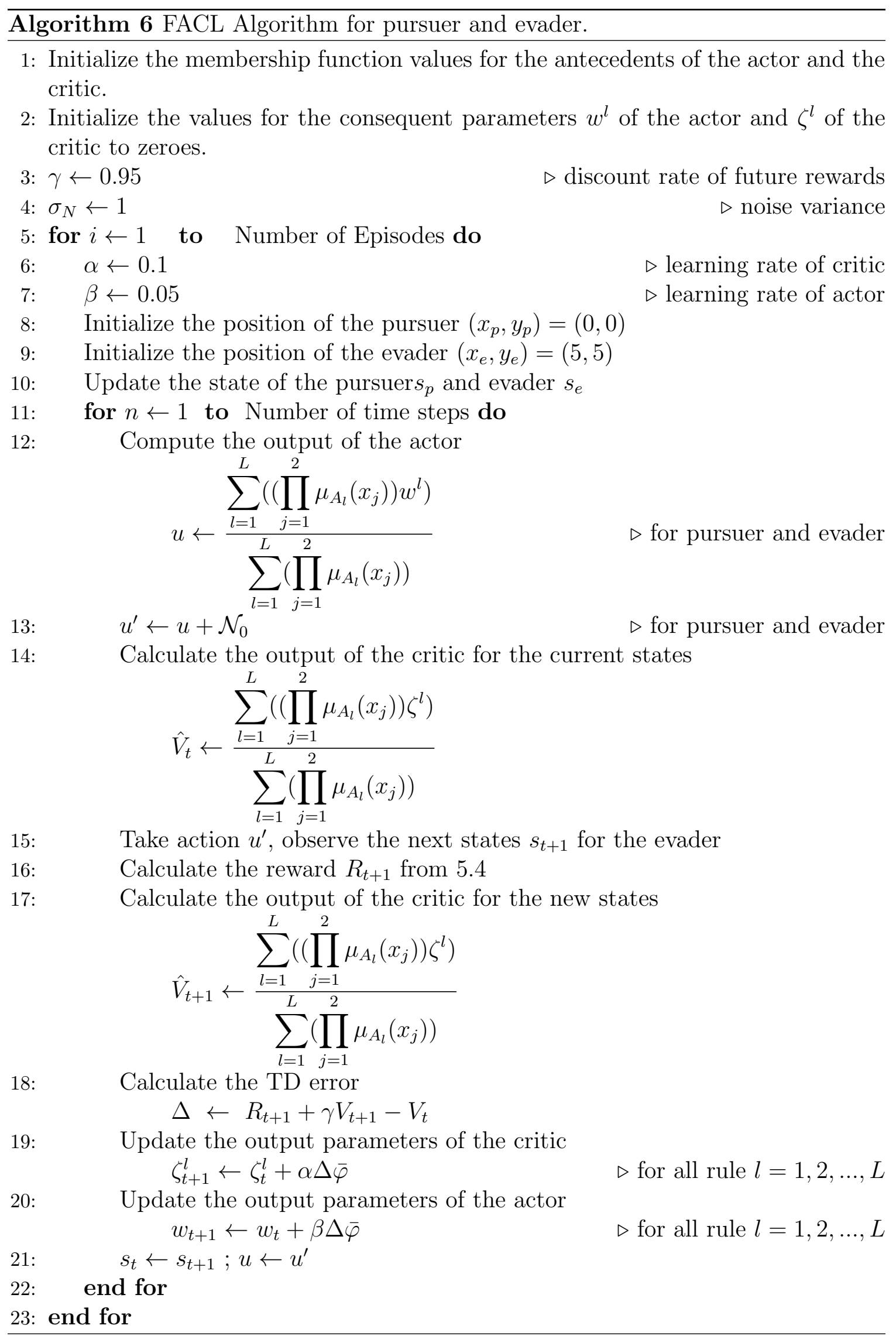


by observing if the evader learns to escape from the pursuer. We observe the number of learning episodes it takes for the evader to learn its escape strategy. We know that triangular membership functions require less computation than Gaussian membership functions. Triangular membership functions can also be constructed in a way that only two membership functions overlap and can be activated for any given input. We observe the computation time when using our approach and the QLFIS in [1]

In the game setup, we assume that the speed of the pursuer is twice the speed of the evader, $V_{p}=2 \mathrm{~m} / \mathrm{s}$ and $V_{e}=1 \mathrm{~m} / \mathrm{s}$. The evader is a pedestrian and is able to take sharp turns in any direction making the evader more maneuverable than the pursuer as described in Chapter 4. The pursuer is a car with wheelbase $L_{p}=0.3 \mathrm{~m}$ and maximum steering angle of $u p_{\max } \in[-0.5 \mathrm{rad}, 0.5 \mathrm{rad}]$. The pursuer's minimum turning radius is $R p=0.5491 \mathrm{~m}$ from Equation 4.2 and the capture radius $\ell=0.15 \mathrm{~m}$. The players' parameters were specifically chosen in order to satisfy Isaacs' capture condition given by Equation 4.7 and reiterated here as

$$
\ell / R_{p}>\sqrt{1-\gamma^{2}}+\sin ^{-1} \gamma-1
$$

where $\ell / R_{p}$ is the ratio of the radius of capture to the minimum turning radius of the pursuer, and $\gamma=v_{e} / v_{p}<1$ is the ratio of the evader's speed to the pursuer's speed. If the condition in Equation 5.5 is true, the pursuer will surely capture the evader. If the condition is false, the evader will escape. The evader is captured by the pursuer when the Cartesian distance $D \leq \ell$. We have chosen the game parameters such that there exists an escape strategy for the evader. The evader will escape from the pursuer if the evader can successfully learn its optimal control strategy given in Equation 4.10. The evader's optimal control strategy is to take a sharp turn into the circular region created by the pursuer's minimum turning radius $R_{p}$ when the pursuer 
comes within a threshold distance of $D \leq R_{p}$.

\subsubsection{Homicidal chauffuer game using QLFIS algorithm in [1]}

We start by using the QLFIS algorithm (Algorithm 5) to train both pursuer and evader simultaneously as it was done in [1]. However, we focus on the performance of the evader. The two inputs to the pursuer's FIS are the heading error $\phi$ and its rate of change $\dot{\phi}$. The two inputs to the evader's FIS are the line-of-sight (LOS) $\psi$ and the Cartesian distance $D$. Three Gaussian membership functions are created over both input spaces of the pursuer and evader (shown in Figure 5.1). The equation for Gaussian membership functions is given as

$$
\mu_{A_{l}}\left(x_{i}\right)=\exp \left(-\left(\frac{x_{i}-c_{i}^{l}}{\sigma_{i}^{l}}\right)^{2}\right)
$$

where the Gaussian MF parameters, the mean $c$ and the standard deviation $\sigma$, are the input parameters to be tuned. Both pursuer and evader's FIS thereby have nine rules $(3 \times 3=9)$ each. Also, two input parameters and one output consequent parameter are updated for each rule at every time step. A total of 27 learning parameters are updated for both FIS and FLC for both the pursuer and the evader in [1]. Simulation results from [1] showed that the use of eligibility traces did not significantly improve the evader's learning. Therefore, we do not use eligibility traces in this simulation. We run the simulation 20 times with a training period of 1000 episodes and 600 time steps per episode. The sampling time $T=0.1$, therefore the total game duration is 60 seconds. We observe the learning speed of the evader and the computation time of the algorithm.

The evader learns its control strategy after about 927 episodes and consistently escapes in subsequent episodes. We timed the computation of the QLFIS algorithm 


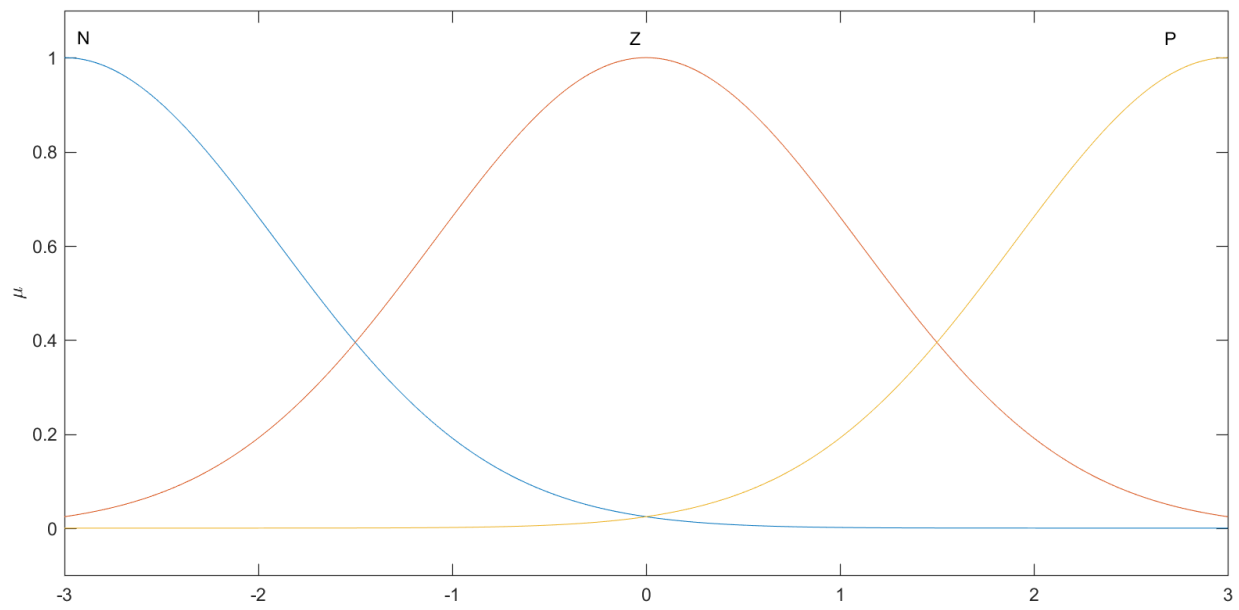

(a) Gaussian membership functions for the pursuer's input $\phi$ and $\dot{\phi}$ and the evader's input $\psi$ before learning

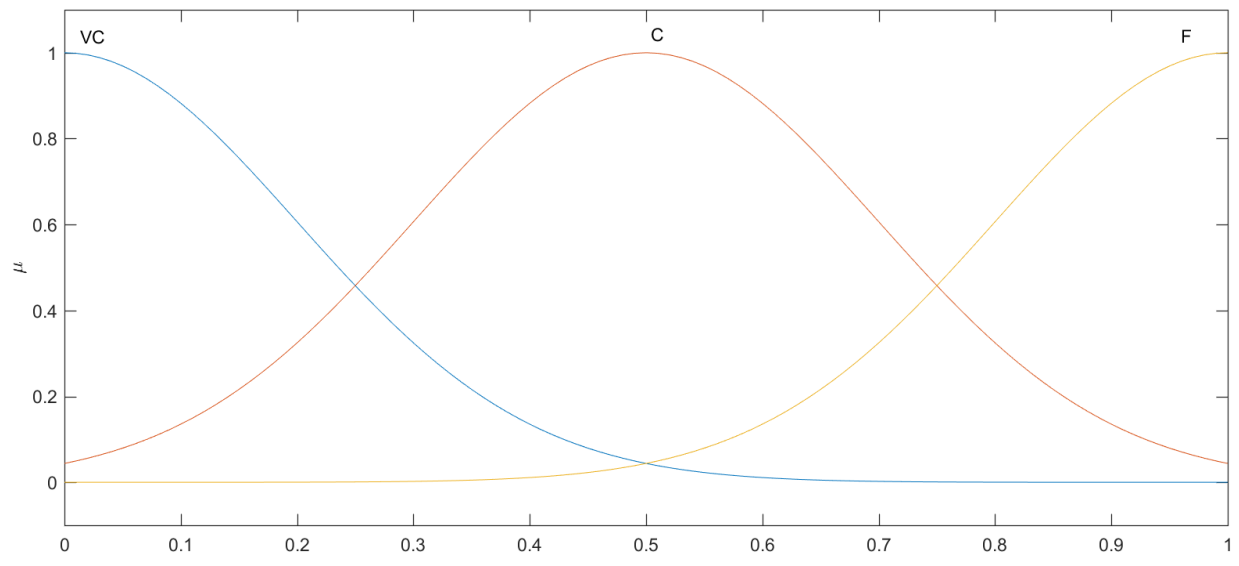

(b) Gaussian membership functions for evader's input $D$ before learning

Figure 5.1: Gaussian membership function of pursuer and evader before learning 
at the terminal episode. The computation time taken to run the last 100 episodes was an average of 40.5 seconds. The evader's input parameters after learning are shown in Figure 5.2. If we look closely at the evader's membership functions before and after training, we notice that the membership functions of distance $D$ (Figure 5.1(b) and Figure 5.2(b)) changed significantly more than the membership functions of $\phi$ (Figure 5.1(a) and Figure 5.2(a)). This is because the evader's control strategy of making a sharp turn is dependent on how close the pursuer is to the evader. Thereby, confirming the fact that distance $D$ is very important to the evader.

\subsubsection{Homicidal Chauffuer Game using the FACL Algorithm}

In this simulation, we use triangular membership functions for both pursuer and evader. The input to the pursuer's FIS is the heading error $\phi \in[-\pi, \pi]$ between the pursuer and the evader. We create five membership functions over the pursuer's universe of discourse shown in Figure 5.3. The pursuer maintains the same number of membership function for all simulations. We simulate three cases of the homicidal chauffeur game. The game parameters of the pursuer and evader are the same for all three cases. However, the cases differ from one another in terms of the number of membership functions covering the evader's input space and the range of the input space itself. We investigate whether increasing the number of membership functions over the evader's input space affects the evader's learning speed and how well it learns. We run the game for each case for 1000 training episodes. The termination time for each episode is 600 time steps (i.e 60 seconds) with a sampling time of $T=0.1 \mathrm{sec}$. The game terminates when the pursuer captures the evader (i.e when $D \leq \ell$ ) or when the total duration of the game is elapsed (i.e after 60 seconds). 


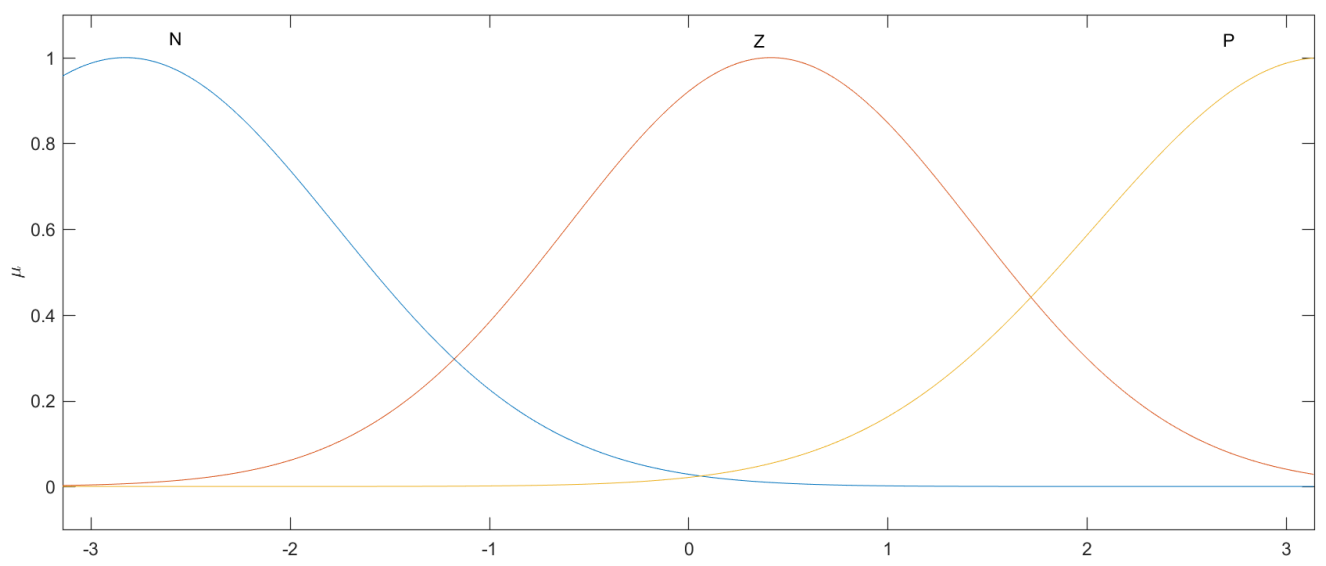

(a) Evader's Gaussian membership functions for $\psi$ after learning

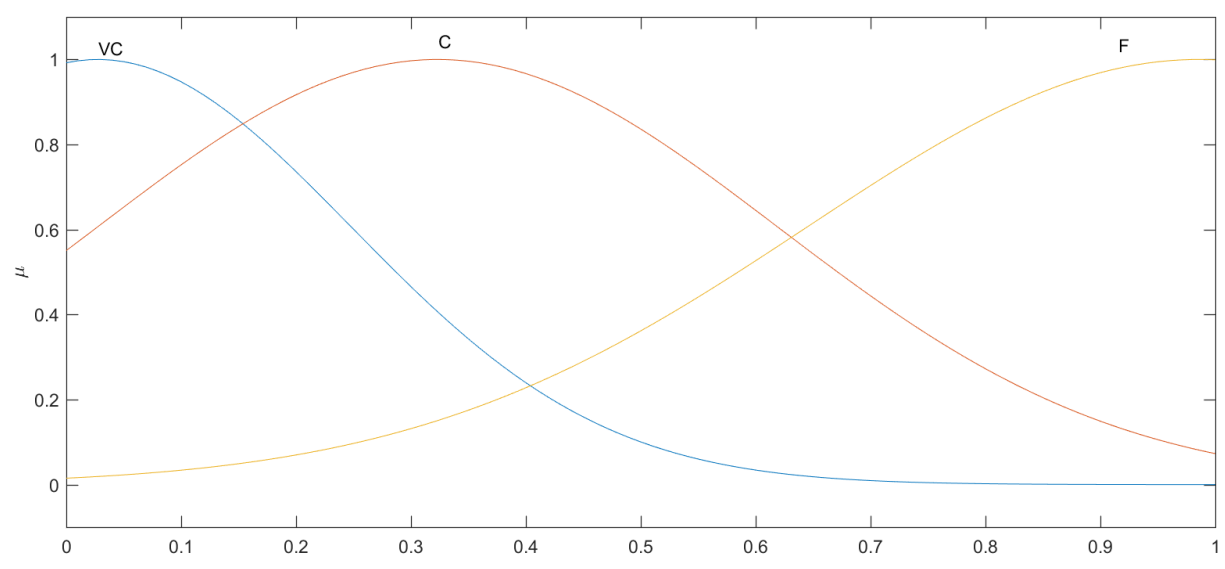

(b) Evader's Gaussian membership functions for $D$ after learning

Figure 5.2: Gaussian membership functions of evader after learning with the QLFIS algorithm in $[1,4]$

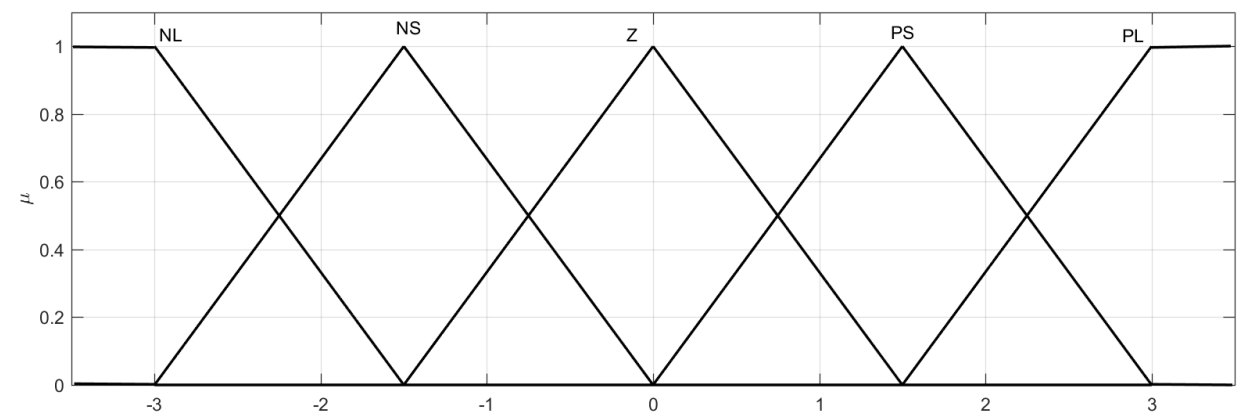

Figure 5.3: Pursuer's membership functions for all games 


\section{Case 1}

In this scenario, three triangular membership functions are created over the evader's input spaces $\psi \in[-\pi, \pi]$ from (4.4) and Cartesian distance $D \in[0,1]$ from 5.2, as shown in Figure 5.4. The three membership functions for the two inputs result in $L=3 \times 3=9$ rules each for the actor and the critic. The linguistic terms for the membership functions over input $\psi$ are: Negative $(N)$, Zero $(Z)$, and Positive $(P)$. The linguistic terms for the membership functions over input $D$ are: Very Close $(V C)$, Close $(C)$ and Far $(F)$. We note, from the structure of the triangular membership functions of the evader's inputs in Figure 5.4, that any input value in $\psi$ or $D$ can only belong to at most two membership functions. Therefore, the FIS can only have at most four rules fire for both inputs even if we add more membership functions. Hence, only the four output parameters of the rules that fire need to be updated at every time step. In [1,4], where Gaussian membership functions were used, all rules can fire for a given input.

The pursuer's initial position is set at the origin $\left(x_{p}=0, y_{p}=0\right)$, with an initial heading angle of $\theta=0 \mathrm{rad}$. The evader's position is initialized at $\left(x_{e}=5, y_{e}=5\right)$. The membership degree of inputs that exceed the limits of $D \in[0,1]$ saturates at $\mu_{D}=1$. Therefore, we can initialize the evader at any distance away from the pursuer. We run the game 20 times with a learning period of 1000 episodes and 600 time steps per episode. We averaged the results over 20 trials and observe the performance of the evader.

At the early learning episodes, the pursuer always captures the evader. After approximately 200 episodes, the evader made some successful maneuvers and escapes. Due to the evader's exploration of the action space, the evader manages to delay capture in some episodes. For example, Figure 5.5 shows that during episode 300 


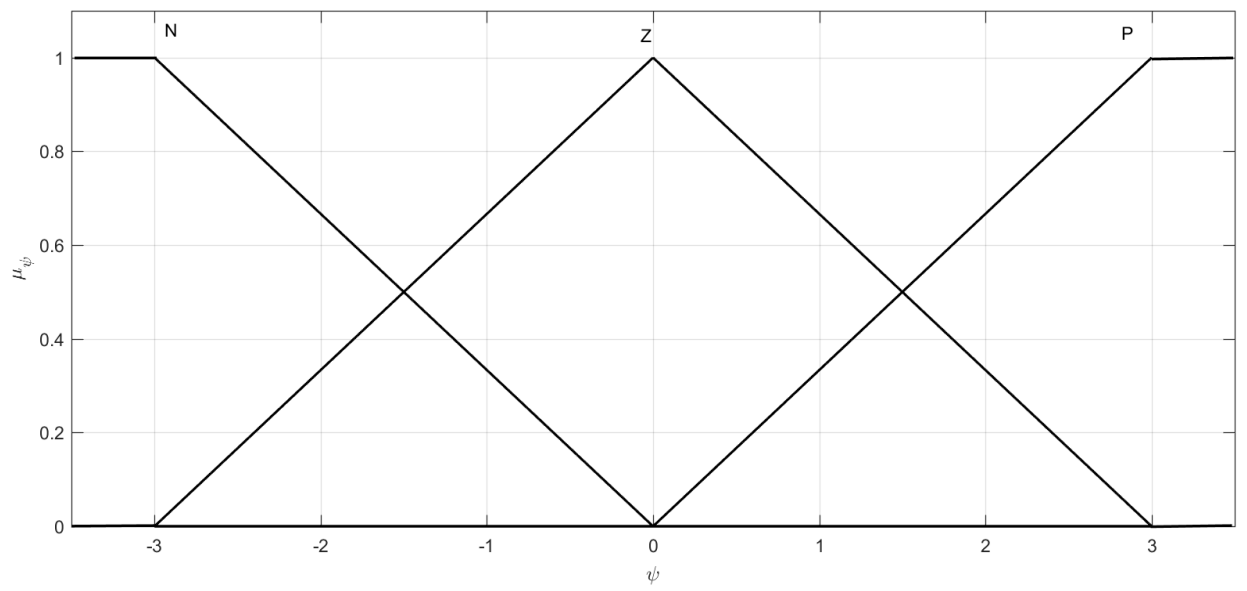

(a) The evader's LOS $\psi$ for the homicidal Chauffeur game



(b) The evader's distance $D$ for the homicidal Chauffeur game case 1

Figure 5.4: The evader's membership functions for Case 1 of the homicidal chauffeur game 
the evader made some maneuvers (i.e. took sharp turns), delayed capture but did not escape. The evader manages to escape in an average of 17 episodes out of 1000 episodes with an average capture time of 14.50s. At episode 1000, the evader gets captured as shown in Figure 5.6. The evader was unable to learn its control strategy by the end of the training period. The evader performed poorly when compared to the evader's performance when using the QLFIS algorithm in [1] where the evader is able to escape completely and consecutively after 927 episodes. We recall from

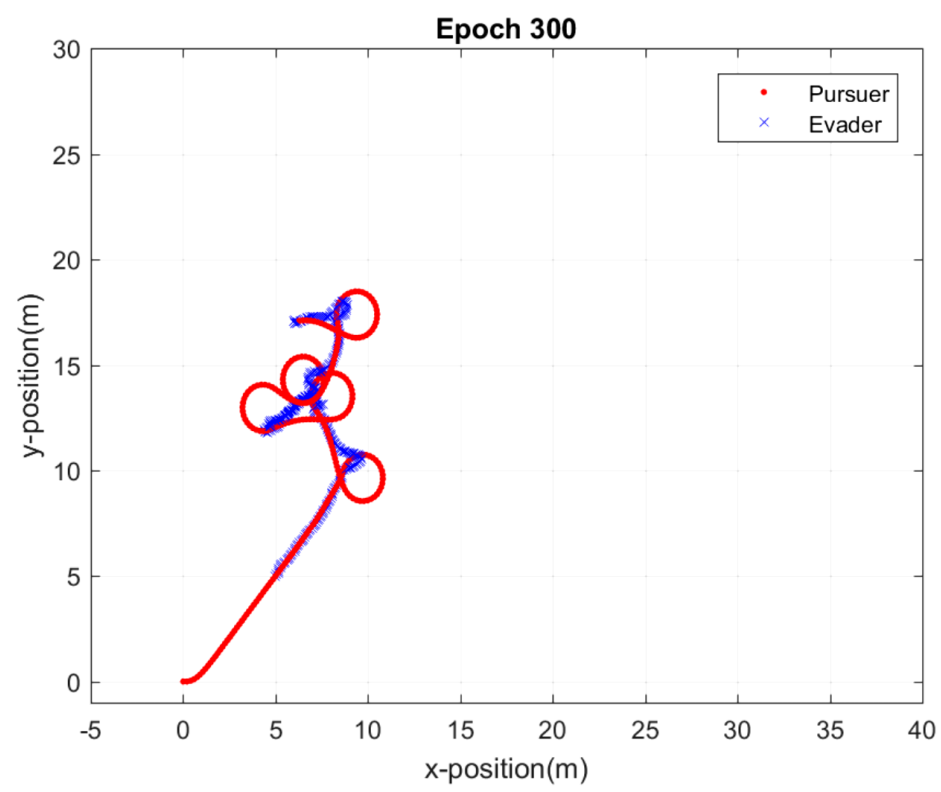

Figure 5.5: Evader increases capture time at episode 300 in the homicidal chauffeur game Case 1

Chapter 4 that the evader's escape strategy is to take a sharp turn into the circular region created by the minimum turning radius $R_{p}$ of the pursuer (Fig. 4.2), when the Cartesian distance $D$ between the pursuer and evader is such that $D \leq R_{p}$. Therefore, distance $D=R_{p}$ is very important to the evader's control strategy since the evader needs to know when to turn. We observe the rules that are fired when $D=R_{p}$. We recall that $R_{p}=0.5491 \mathrm{~m}$ given the pursuer's parameters and that $D=R_{p}$ belongs to 




Figure 5.6: Evader captured at episode 1000 in the homicidal chauffeur game Case 1

the membership function $C$ as shown in Figure 5.6. We want the rules associated with the membership function $C$ to fire very often. We believe that if the rules fire often, those states are visited often and the critic parameters of those rules will converge to correct values. Table 5.1 shows the rules that fire when $D=R_{p}$ and how many times they fire in 1000 episodes and averaged over 20 trials.

Table 5.1: Summary of the average number of times the rule with premise $C$ (i.e $D=R_{p}$ ) fire during 1000 episodes of the homicidal chauffeur game case 1

\begin{tabular}{|c|c|c|c|}
\hline$d \lambda$ & $\mathrm{N}$ & $\mathrm{Z}$ & $\mathrm{P}$ \\
\hline $\mathrm{C}$ & 2890 & 15278 & 12388 \\
\hline
\end{tabular}

We run a simulation of the homicidal chauffeur game using the time optimal strategies of both players as described in Chapter 4. We observed that the maximum reward value the evader can receive in an episode of the homicidal chauffeur game is 0.7155. This maximum reward value is received when the evader's distance $D=R p$ 
from the pursuer, and the evader takes a sharp turn to temporarily increase the distance between itself and the pursuer. We recall that the job of the critic is to estimate the state-value function $V$ using the temporal difference error $\Delta_{t}$ in 3.17. Since we use a discount factor of $\gamma=0.95$, the maximum value that can be achieved at state $D=R_{p}$ is 1.3952. For example, if the evader's input states $[\psi, D]=\left[0.2, R_{p}\right]$, one of the four rules that fire is the rule " $\psi$ is $N$ and $D$ is $C$ ". The firing strength of that rule will be $0.6 \times 0.9=0.54$. The firing strengths of all the rules that fire sum up to one. Therefore, $\sum_{l=1}^{9} \bar{\varphi}_{t}^{l}=1$ from 3.25 which is rewritten in 5.7 .

$$
\hat{V}_{t}=\sum_{l=1}^{L} \bar{\varphi}_{t}^{l} \zeta_{t}^{l}
$$

The critic's output parameter becomes $\zeta_{t}^{l}=1.3952$, which is the value the critic parameter's should converge to for rules that fire when $D=R_{p}$. Figure 5.7 shows the critic parameters of rules with premise $C\left(D=R_{p}\right)$. We observe from Figure 5.7 that only one of the critic's parameters achieved a convergence value of 0.7577 . The other two parameters never converge even after an extended training period of 10,000 episodes. The computation time of the algorithm was recorded during the last 100 episodes and it was an average of 20.02 seconds which is lesser than the QLFIS algorithm in [1]. The example given in Chapter 2 showed that we can achieve a better approximation in a fuzzy inference system with more membership functions. The evader was unable to learn its control strategy using only three membership functions for both input space. 


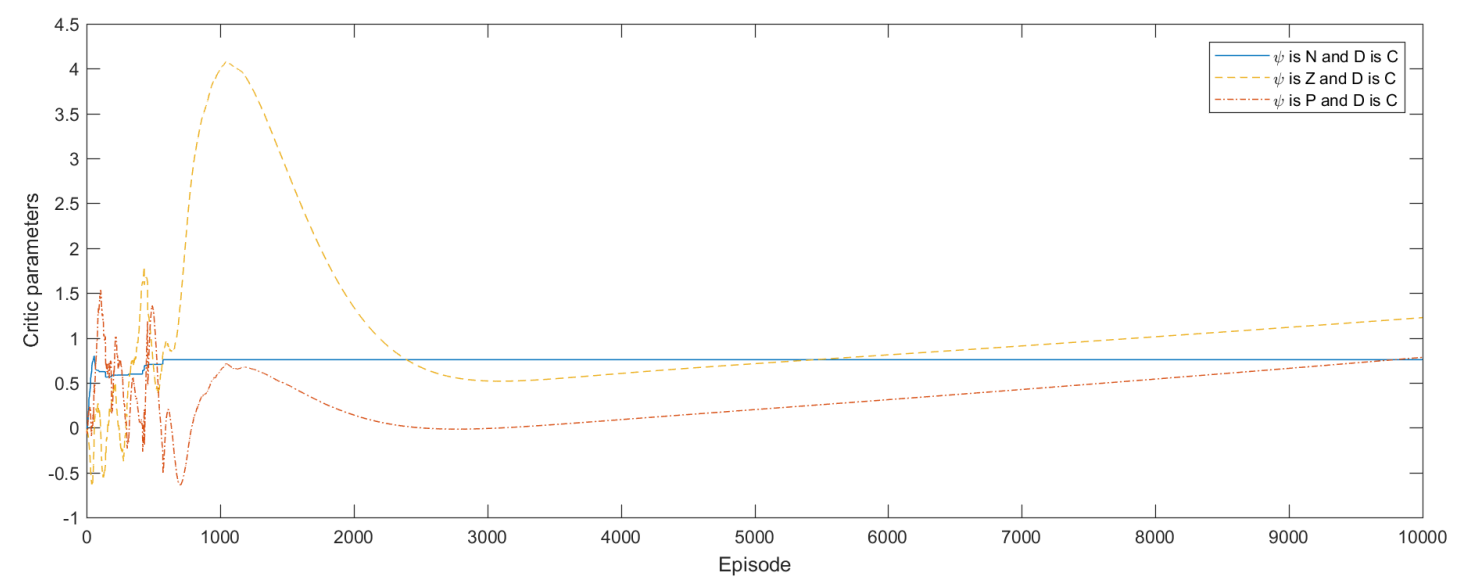

Figure 5.7: Critic's output parameters of rules with premise $C$ in the homicidal chauffeur game case 1

\section{Case 2}

We aim to improve the performance of the evader in case 1 by including additional rules. We create more rules by increasing the number of membership functions covering the input space of $D$. We increase only the number of membership functions over input space $D$ because the distance between the pursuer and evader is important for the evader to know when to turn. In this scenario, we create 10 membership functions over input space $D$ of the evader as depicted in Figure 5.8. The number of membership functions over the evader's second input space $(\psi)$ remains the same as case 1 shown in Figure 5.4(a). The total number of rules in this game for both actor and critic is $3 \times 10=30$, therefore there are 30 parameters to be updated for each rule. We recall that because of the structure of the triangular membership functions, only a maximum of four rules can fire for any given pair of inputs in a two input FIS. Therefore, increasing the number of membership functions does not increase the number or rules the evader's FIS is required to update. Only a maximum of four parameters will be updated at every time step in this game. Unlike in [1], where a 
total of 27 parameters are updated at every time step despite using 3 Gaussian membership functions for two inputs. The alphabets that make up the linguistic terms for the evader's input $D$ are, $E$ : Extremely, $V$ : Very, $C$ : Close and $F$ : Far. For example, the term $V V V C$ means "Very Very Very Far".

All other game parameters remain the same as in case 1 . We expect the evader to learn to turn at the rule with premise $D=F$, where $F$ is the linguistic term "Far" for $D=R_{p}$. We run the simulation 20 times and averaged the results. The simulation



Figure 5.8: The evader's membership functions for distance $D$ for the homicidal Chauffeur game case 2. Note the location of $R_{p}$

shows that the evader was able to delay its capture by approximately $28.4 \mathrm{sec}$ at episode 400. The evader was able to increase the total number of episodes it escapes from the pursuer to an average of 420 episodes out of 1000 and finally learned to escape consistently at approximately episode 780 . Figure 5.9 and 5.10 show the players at episodes 400 and 1000 respectively. In this case, the evader learns to make sharp turns and escape the pursuer in fewer learning episodes than the method in [1] where it takes 927 episodes for the evader to learn to escape. The computation time of the algorithm recorded during the last 100 episodes is about 20.8 seconds which 
is slightly higher than case 1 but still less than the QLFIS algorithm in [1]. Figure 5.8 shows that the membership function over the input $D=R_{p}$ is associated with the linguistic term $F$ in the input space $D$. The increase in membership functions enables the evader to be able to detect distance $D$ state values to a finer degree and notice changes in the input states with more precision than in case 1. Therefore, the rules associated with $F$ fire more in this scenario than in case 1 which reflects in Table 5.2. The critic's output parameters related to the rules with premise $F$, from Figure 5.11, all converge to values close to the maximum possible value of 1.3952 . We run the simulations for an extended learning period of 10000 episodes to to observe whether the parameters converge. From Figure 5.11, rules: " $\psi$ is $N$ and $D$ is $F$ ", " $\psi$ is $Z$ and $D$ is $F$ ", " $\psi$ is $P$ and $D$ is $F$ " converge to the values 1.2160, 1.3130, 1.1091 respectively. 




Figure 5.10: Evader escapes at episode 1000 in the homicidal chauffeur game case 2

Table 5.2: Summary of the average number of times the rule with premise $F$ (i.e $D=R_{p}$ ) fired during 1000 episodes of the homicidal chauffeur game case 2

\begin{tabular}{|c|c|c|c|}
\hline$d \lambda$ & $\mathrm{N}$ & $\mathrm{Z}$ & $\mathrm{P}$ \\
\hline $\mathrm{F}$ & 11176 & 17901 & 16725 \\
\hline
\end{tabular}






Figure 5.11: Critic's output parameters for rules with premise $F$ in the homicidal chauffeur game case 2

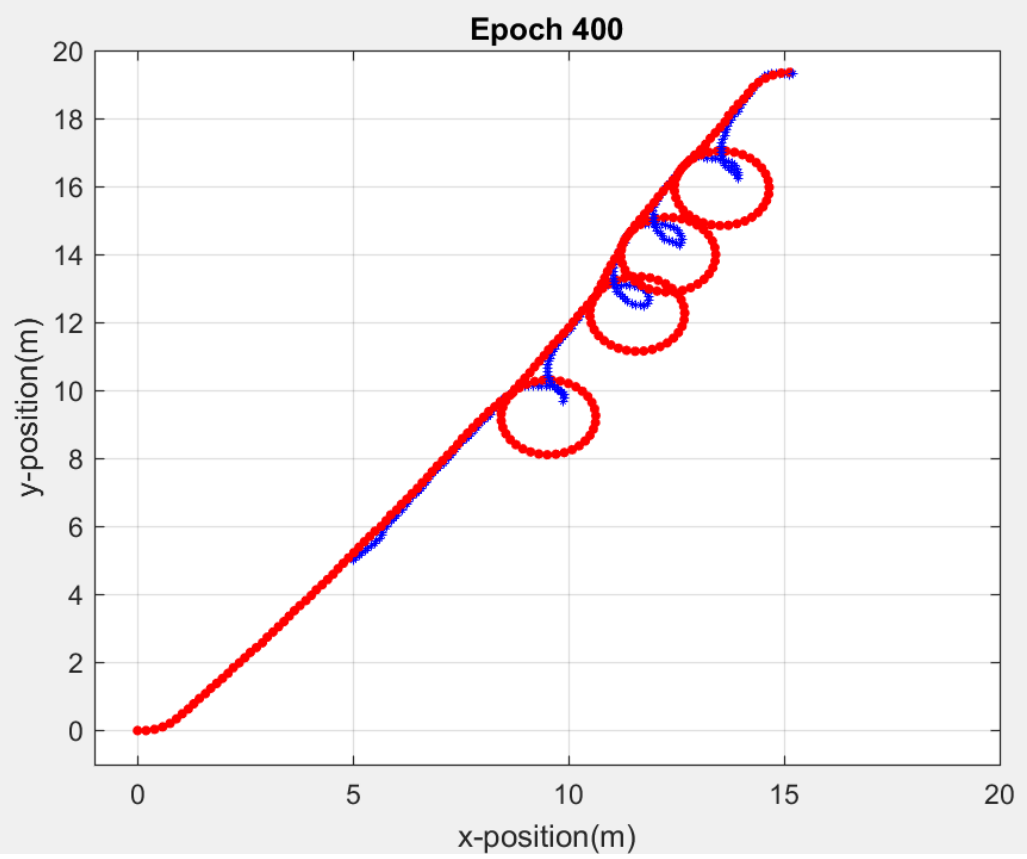

Figure 5.9: Evader increases capture time at episode 400 in the homicidal chauffeur game case 2 


\section{Case 3}

In this game, we have the same number of membership functions for the evader's input $D$ as we had in case 2 . We want the evader to learn to make a sharp turn immediately when $D=R_{p}$. We investigate whether we can improve the learning speed of the evader any further. We decrease the range of the universe of discourse $D$ from $[0,1]$ to $[0.5,1]$. We note that any distance $D \leq 0.5$ would have a membership degree $\mu_{D}=1$ as shown in Figure 5.13. This approach should force the evader to learn to take a sharp turn at the minimum distance of $D=0.5$. In this case, the membership functions densely fill the narrow region of discourse. This should have the same effect as using more membership functions as shown in the example in Chapter 2. All other game and players' parameters remain the same as in case 1 and case 2. We run the simulation 20 times and averaged the results. The evader was able to escape and make successful maneuvers in an average of 835 out of 1000 episodes making its first successful escape by episode 100. The evader learned to escape consistently in successive episodes after approximately 450 episodes.

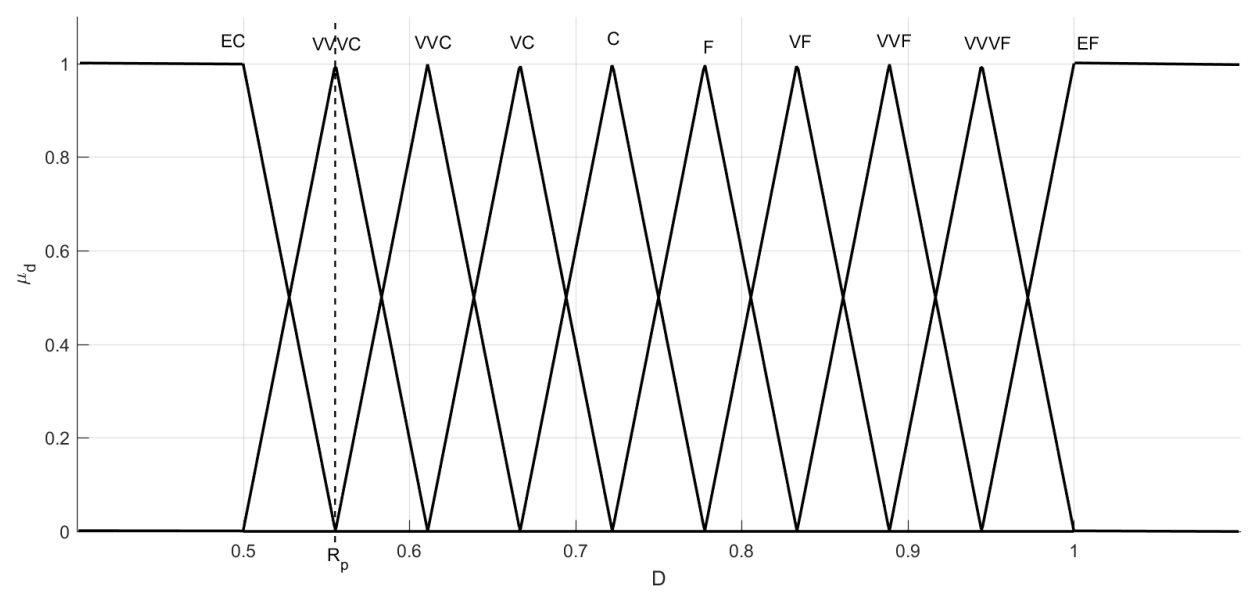

Figure 5.12: The evader's membership functions for $D$ in the homicidal chauffeur game case 3 


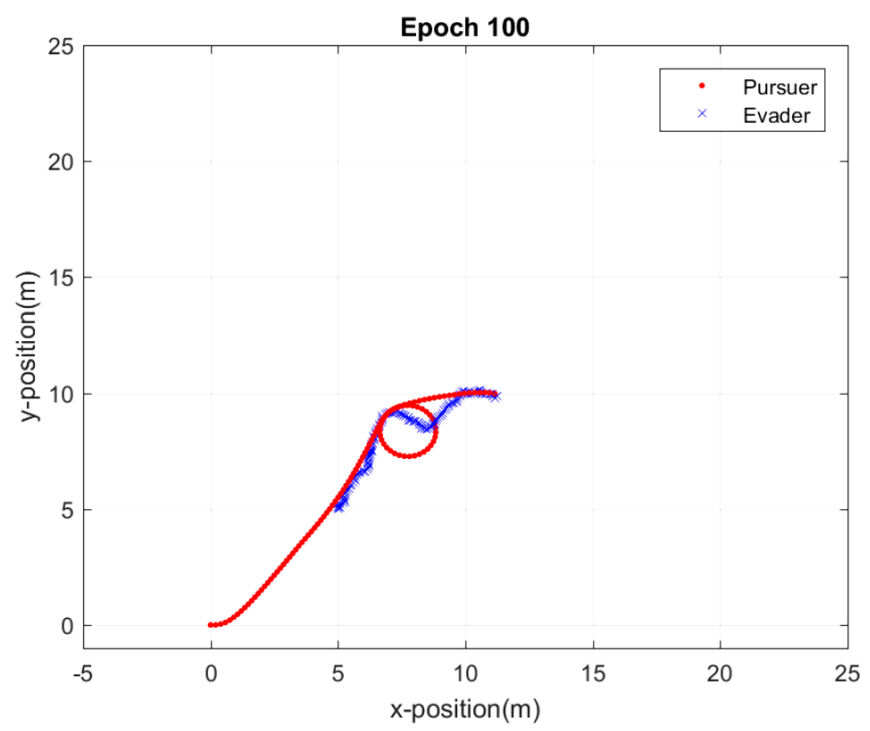

Figure 5.13: Evader increases capture time at episode 100 in the homicidal chauffeur game case 3

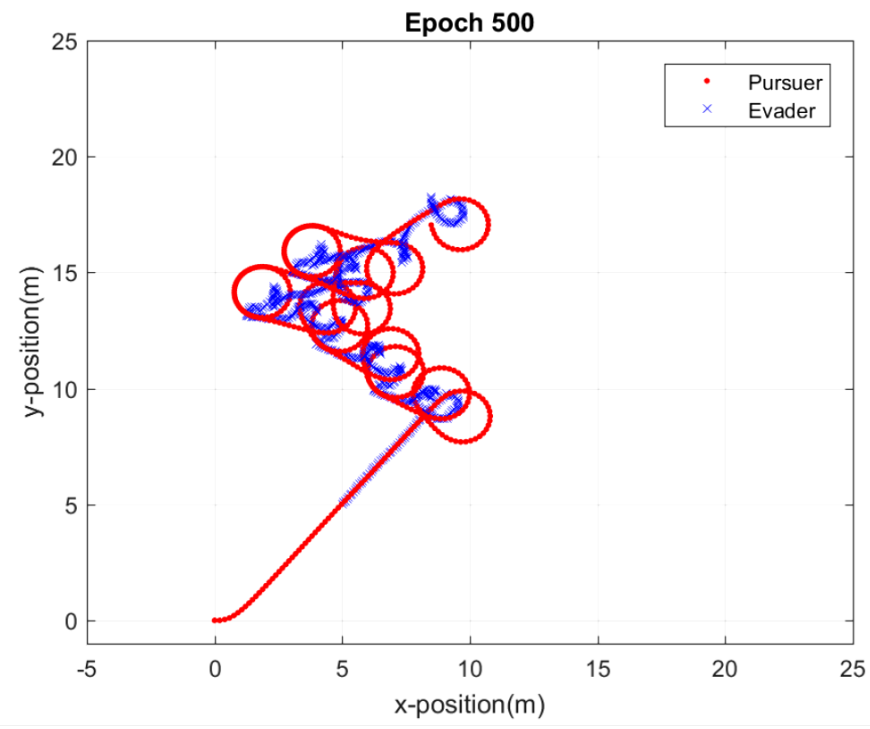

Figure 5.14: Evader escapes at episode 500 in the homicidal chauffeur game case 3

Table 5.3 shows how often the rules at $D=R_{p}$ fire. In this case, $R_{p}$ is represented with the membership function " $V V V C$ ". We observe that the rules at " $D$ is $V V V C$ " fire more often in this case than in cases 1 and 2. This is because we eliminated all 
Table 5.3: Summary of the average number of times the rule with premise $V V V C$ (i.e $D=R_{p}$ ) fired during 1000 episodes of the homicidal chauffeur game case 3

\begin{tabular}{|c|c|c|c|}
\hline$\lambda$ & $\mathrm{N}$ & $\mathrm{Z}$ & $\mathrm{P}$ \\
\hline $\mathrm{VVVC}$ & 28507 & 65,544 & 37,037 \\
\hline
\end{tabular}

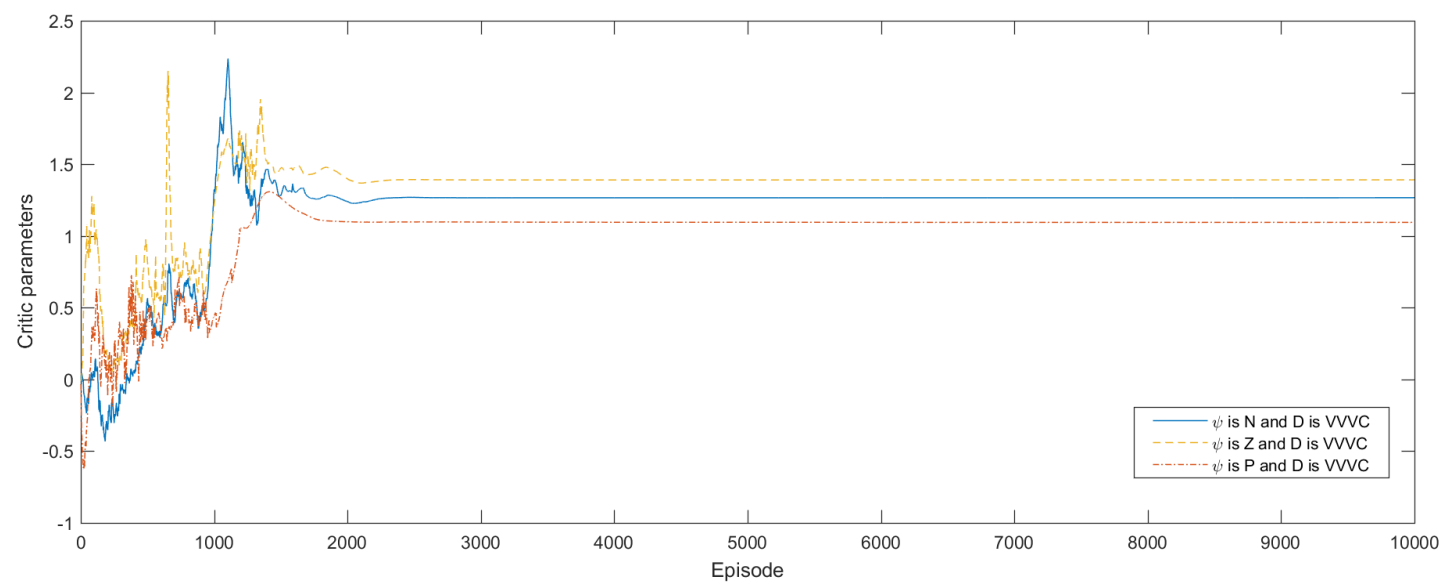

Figure 5.15: Critic's output parameters for rules with premise $V V V C$ in the homicidal chauffeur game case 3 
rules below $D=0.5$ and input variables can be detected with more precision. All the critic's parameters of the rules having the premise "when $D$ is $V V V C$ " converge as seen in Figure 5.15. However, the parameters converge to better values than the values achieved in case 2. From Figure 5.15, the rules: " $\psi$ is $N$ and $D$ is $V V V C$ ", " $\psi$ is $Z$ and $D$ is $V V V C$ ", " $\psi$ is $P$ and $D$ is $V V V C$ " converge to the values 1.2660, 1.4100, 1.1095 respectively. The average of the reward accumulated per episode was averaged over 20 trials for each case. We observe from Figure 5.16 that case 3 accumulated the highest average reward of -0.0261 . The computation time of the algorithm recorded during the last 100 episodes is about 20.8 seconds which is the same as case 2 .

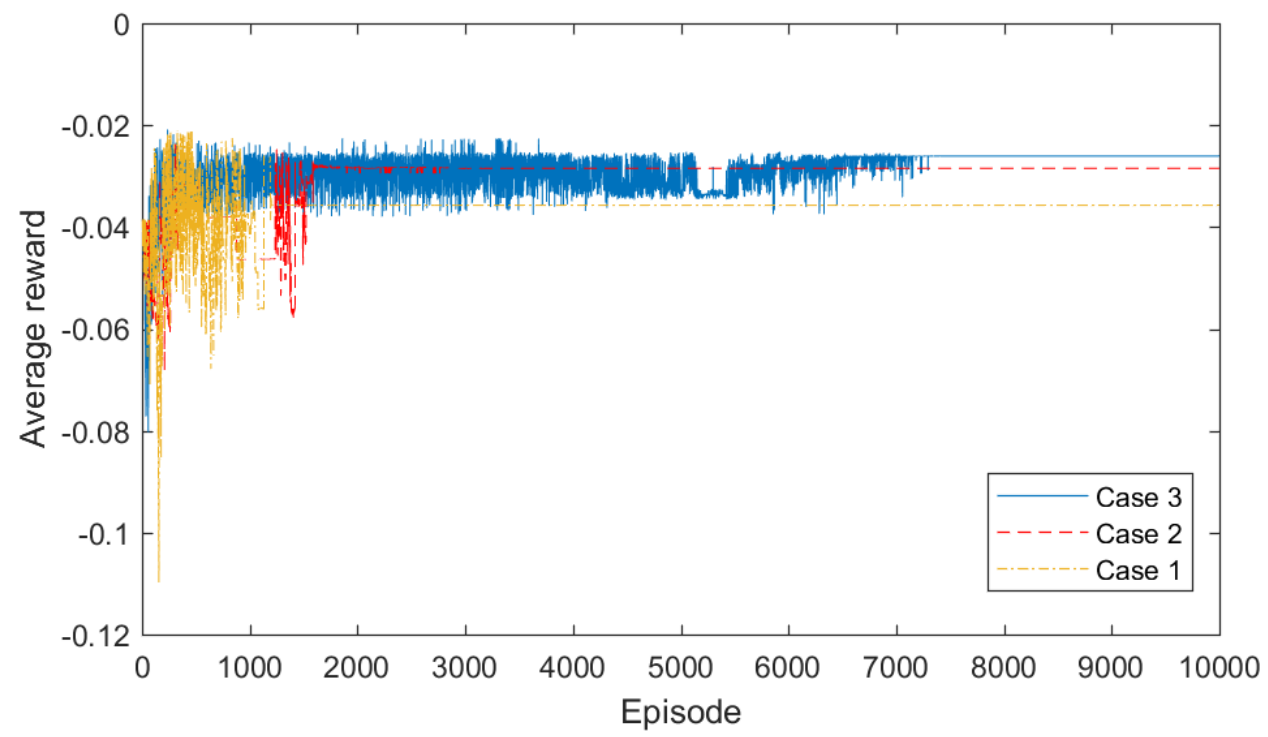

Figure 5.16: Evader's average reward in the homicidal chauffeur game for case 1, case 2 and case 3

Table 5.4 gives the summary of the evader's average learning speed in the three cases of our approach of the homicidal chauffeur game and the average learning speed when we used the QLFIS approach in [1]. 
Table 5.4: Summary of the number of episodes taken for the evader to learn its control strategy and percentage number of times the evader escapes in 1000 episodes in the homicidal chauffeur game

\begin{tabular}{|l|c|l|}
\hline Homicidal chauffeur game & No. of Episodes to learn & $\%$ No. of Escape \\
\hline Case 1 & No learning & $1.7 \%$ \\
\hline Case 2 & 780 & $40.0 \%$ \\
\hline Case 3 & 450 & $83.5 \%$ \\
\hline Approach in [1] & 927 & $10.3 \%$ \\
\hline
\end{tabular}

\subsubsection{Game of Two Cars}

The dynamics of the game of two cars is described in Chapter 4. The parameters of the pursuer are the same as the parameters in our simulation of the homicidal chauffeur game 5.2.1. The evader is modeled as a car with wheelbase $L e=0.3$. The maximum steering angle of the evader is $u_{\text {emax }} \in[-1.2 \mathrm{rad}, 1.2 \mathrm{rad}]$ making the evader more maneuverable than the pursuer. In the game of two cars, there is no theoretical capture condition however, there is a time-optimal strategy proposed by [17] which was described in Chapter 4. The equation of motion of both players is given in Equation 4.12 .

\subsubsection{Game of Two Cars using the QLFIS Algorithm from [1]}

We first play the game using the QLFIS algorithm as described in [1]. The evader's inputs are the angle difference $\theta_{p}-\theta_{e}$ denoted as $\theta_{p e} \in[-\pi, \pi]$ and the distance $D \in[0,1]$. The pursuer's inputs are $\phi \in[-\pi, \pi]$ and $\dot{\phi} \in[-\pi, \pi]$. The membership functions describing both the pursuer's and evader's membership functions are given in Figure 5.1. Although the pursuer and evader learn to play the game simultaneously, our main focus is the evader learning to make sharp turns and eventually escape. We recall that we update both input and output parameters of the players' FIS as it was done in [1]. We run the simulations 20 times, and observe the learning speed of the 
pursuer and the computation time of the algorithm. The membership functions of the evader after learning are shown in Figure 5.17. The computation time recorded for the last 100 episode was an average of 40.5 seconds.

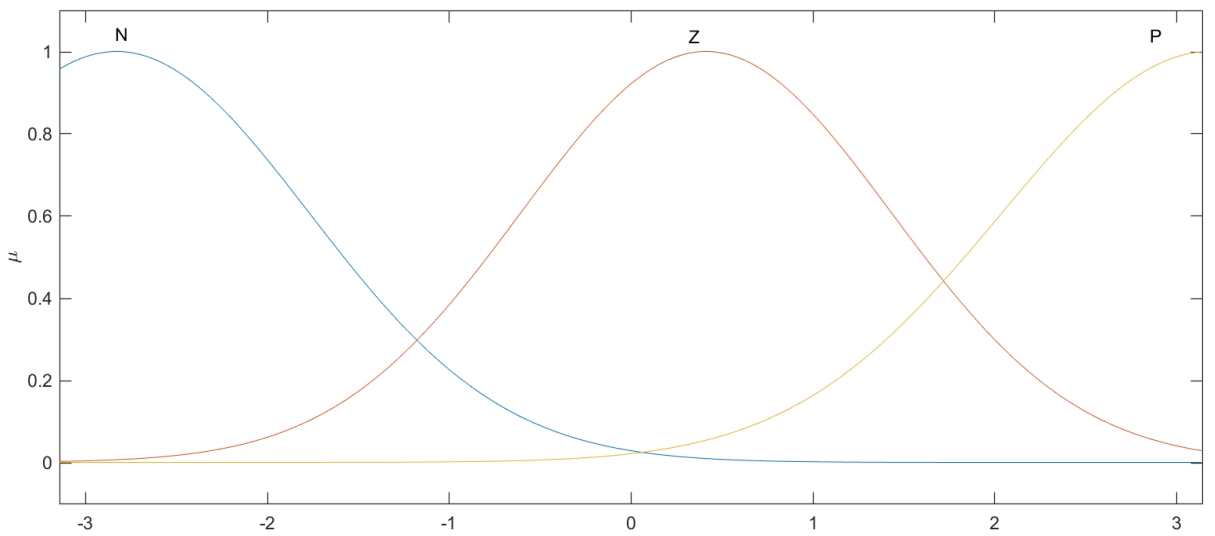

(a) Evader's Gaussian membership functions for $\theta_{p e}$ after learning

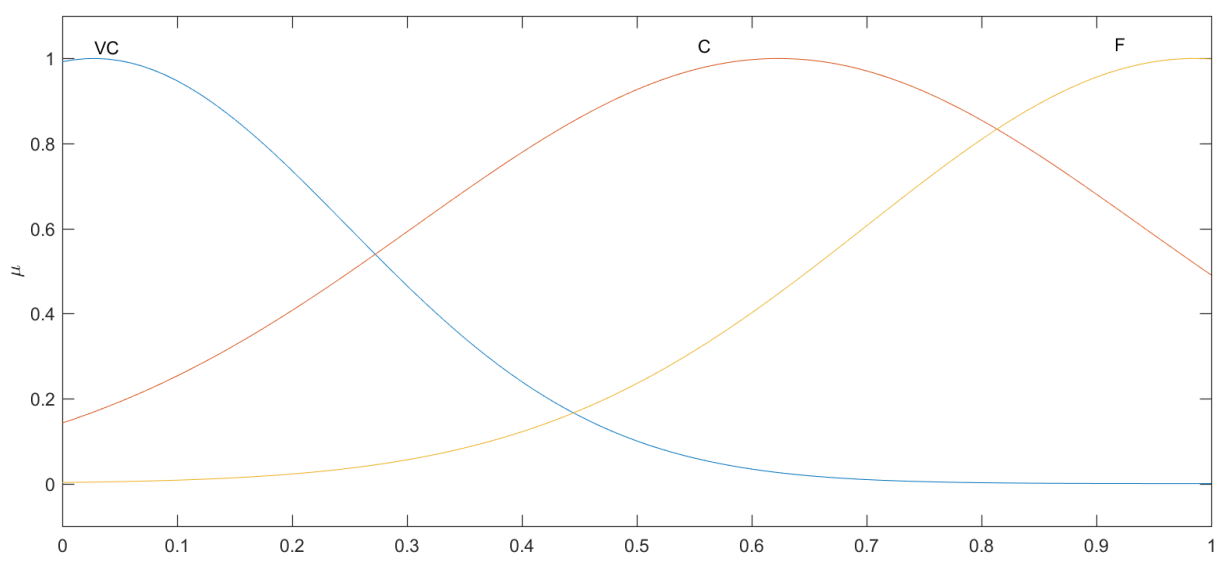

(b) Evader's Gaussian membership functions for $D$ after learning

Figure 5.17: Gaussian membership functions of evader after learning 


\subsubsection{Game of Two Cars using the FACL Algorithm}

Similar to the homicidal chauffeur game in 5.2.1, we use the FACL algorithm to train both pursuer and the evader to learn their time optimal strategies. However, our main focus is on the evader learning to make sharp turns. The pursuer uses the same input and membership functions that were created for the pursuer in the homicidal chauffeur game in Figure 5.3. In this simulation, the evader's inputs are, the angle difference $\theta_{p e} \in[-\pi, \pi]$, and the distance $D \in[0,1]$. We first run a simulation of the game with both players following their optimal strategies. The maximum reward value that can be received by the evader in any episode, given the selected game parameters, is 0.7249 . Just like in the homicidal chauffeur game, the maximum reward is received when $D=R_{p}$ and the evader takes a sharp turn. The maximum state-value for any episode given the selected parameters of this game is 1.4135 .

\section{Case 1}

We first define three membership functions each for both inputs $D \in[0,1]$ and $\theta_{p e} \in[-\pi, \pi]$ of the evader as depicted in Figures 5.4(a) and 5.4(b) respectively. Both the pursuer and the evader learn their control strategy simultaneously using the FACL algorithm given in Algorithm 4. We run the simulation 20 times for 1000 episodes and 600 time steps (i.e 60 seconds) per episode. Figure 5.18 shows the simulation results at episode 1000 where the evader was unable to make any successful maneuvers and was captured in all episodes. In order to trust the values of the updated consequent parameters of the evader's FIS, especially where the evader is expected to turn (i.e $D=R_{p}$ ), we observe how often the rules with premise $C$ fire. We observe from Figure 5.4(b) that the membership function over the area at $D=R_{p}$ has the linguistic term 


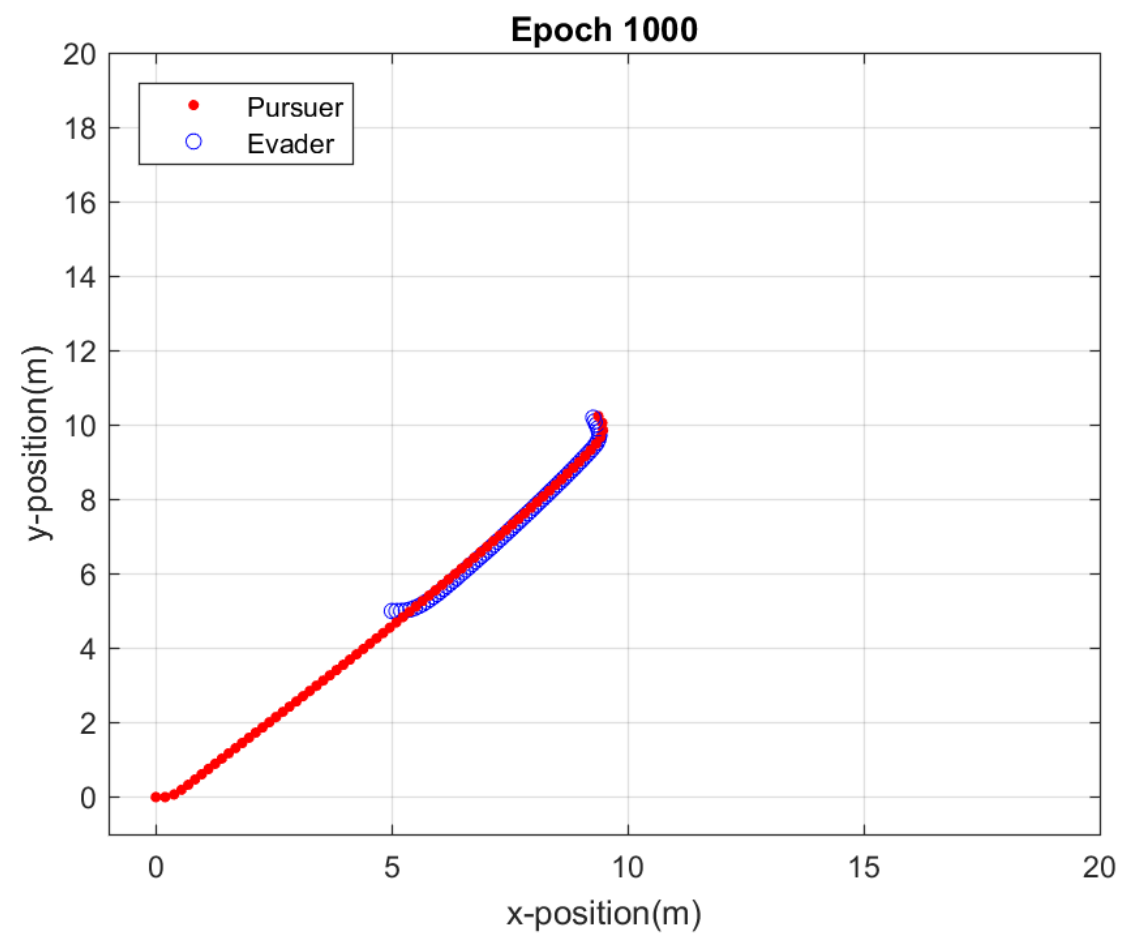

Figure 5.18: Evader is captured at episode 1000 in game of two cars case 1

Table 5.5: The average number of times the rules with premise $C\left(D=R_{p}\right)$ fired during 1000 episodes in game of two cars case 1

\begin{tabular}{|c|c|c|c|}
\hline \multicolumn{1}{|c|}{${ }^{\lambda}$} & $\mathrm{N}$ & $\mathrm{Z}$ & $\mathrm{P}$ \\
\hline $\mathrm{C}$ & 11254 & 13577 & 2323 \\
\hline
\end{tabular}




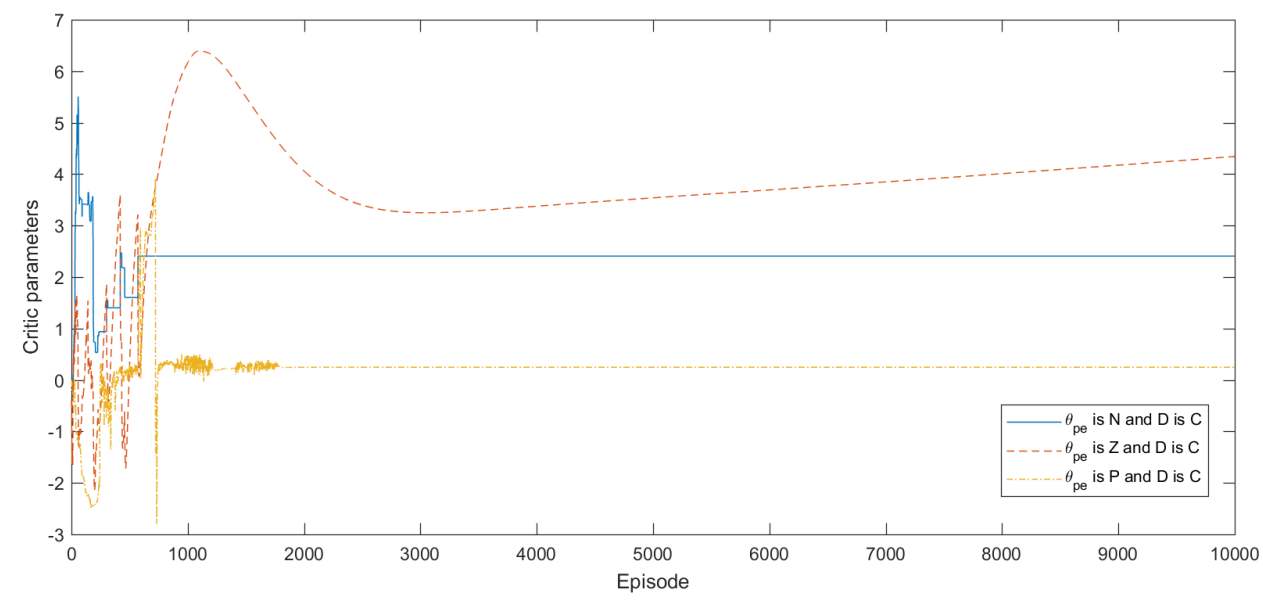

Figure 5.19: Critic's output parameters for rules with premise $C$ in game of two cars case 1

$C:$ Close. We observe how often the rules with premise $C$ is fire in Table 5.5. The evader's critic output parameters for rules with " $C$ " as a premise are shown in Figure 5.19. We observe from Figure 5.19 that only two parameters converge. None of the parameters converge to values that are close to the maximum value of 1.4135 .

\section{Case 2}

To improve the performance of the evader in case 1 , we increase the number of membership functions for $D$ from 3 to 10 membership functions. We use the same membership functions that were created for the evader's distance in case two of the homicidal chauffeur game in Figure 5.8 and observe the number of times the rules associated with $D=R_{p}$ fire. We ran 20 simulations and averaged the results. The simulation results presented in Figure 5.20 show that the evader was able to achieve some successful maneuvers and delay capture by 24.5 seconds at episode 500 . The evader learns to escape consistently after 850 episodes. Figure 5.21 shows the evader escaping at episode 1000. 


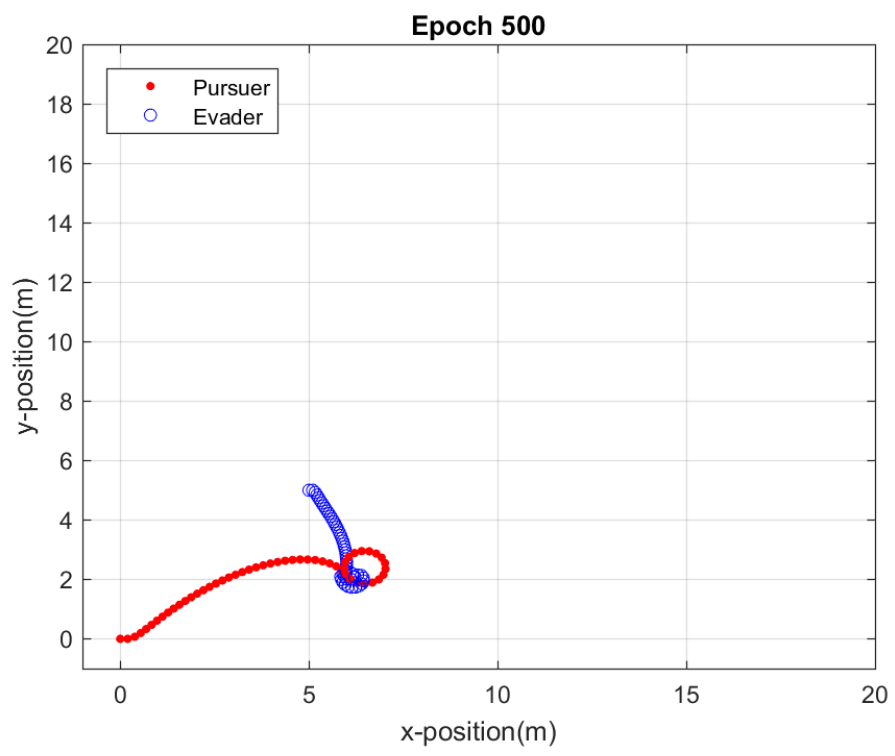

Figure 5.20: Evader is captured at episode 500 in game of two cars case 2

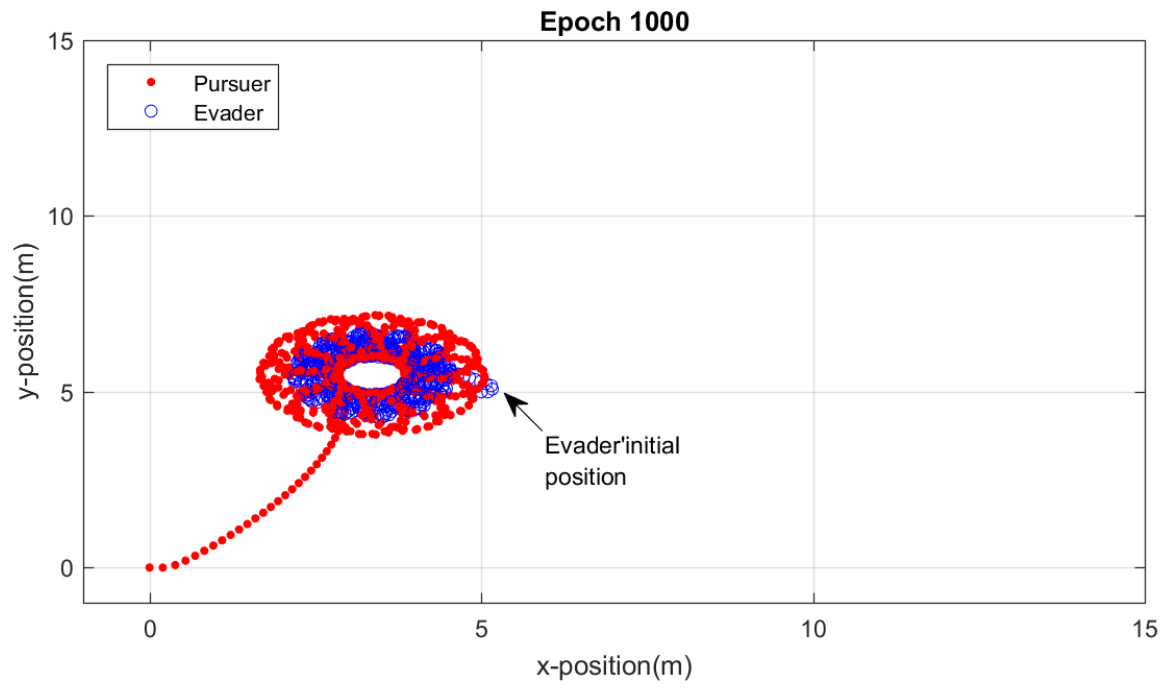

Figure 5.21: Evader is captured at episode 1000 in game of two cars case 2 
The linguistic term associated with $D=R_{p}$ is $F: F a r$. We observe the number of times the rules with premise $F$ fire in Table 5.6. We observe that the rules fire more often because the evader turns more often to escape. The performance of the evader was also better than the evader in case 1. Figure 5.22 shows the critic's parameters of the rules having premise $F$ after an extended play of 10000 episodes. We observe that all the three parameters converge. From Figure 5.22, the rules: $\psi$ is $N$ and $D$ is $F, \psi$ is $Z$ and $D$ is $F, \psi$ is $P$ and $D$ is $F$ converge to the values 1.0820, 1.3031, 1.0515 respectively.

Table 5.6: The average number of times the rules with premise $F\left(D=R_{p}\right)$ fired during 1000 episodes in game of two cars case 1

\begin{tabular}{|c|c|c|c|}
\hline$d$ & $\mathrm{~N}$ & $\mathrm{Z}$ & $\mathrm{P}$ \\
\hline $\mathrm{F}$ & 29156 & 36028 & 15849 \\
\hline VF to EF & 0 & 0 & 0 \\
\hline
\end{tabular}

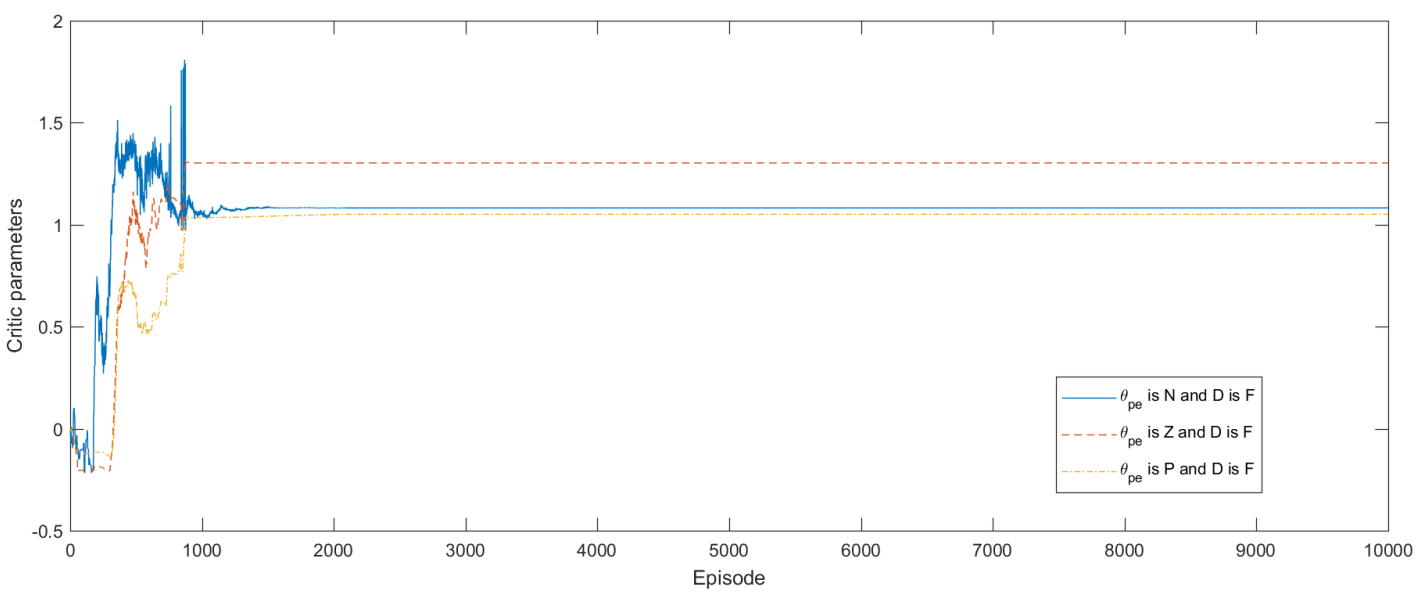

Figure 5.22: Critic's output parameters for rules with premise $F$ in game of two cars case 2 


\section{Case 3}

In this scenario, we decrease the range of the universe of discourse from $D \in[0,1]$ to $D \in[0.5,1]$ as shown in Figure 5.12. We maintain the same structure and number of fuzzy membership functions created in case 2. The number of membership functions for input $\psi$ remains unchanged from case 1 and case 2 . The simulation results in Figure 5.23 show that the evader was able to make some maneuver and delay capture for 26.2 seconds after 100 episodes. The evader learns to successfully escape after about 700 episodes. Figure 5.24 shows the pursuer evader trajectory at episode 1000 . The rules that fire when the distance $D=R_{p}$ is at $V V V C$ are given in Table 5.7. We observe that the rules at $D=R_{p}$ fire more frequently than in case 1 and case 2 just like in the case 3 of homicidal chauffeur game. The rules that fire when the distance

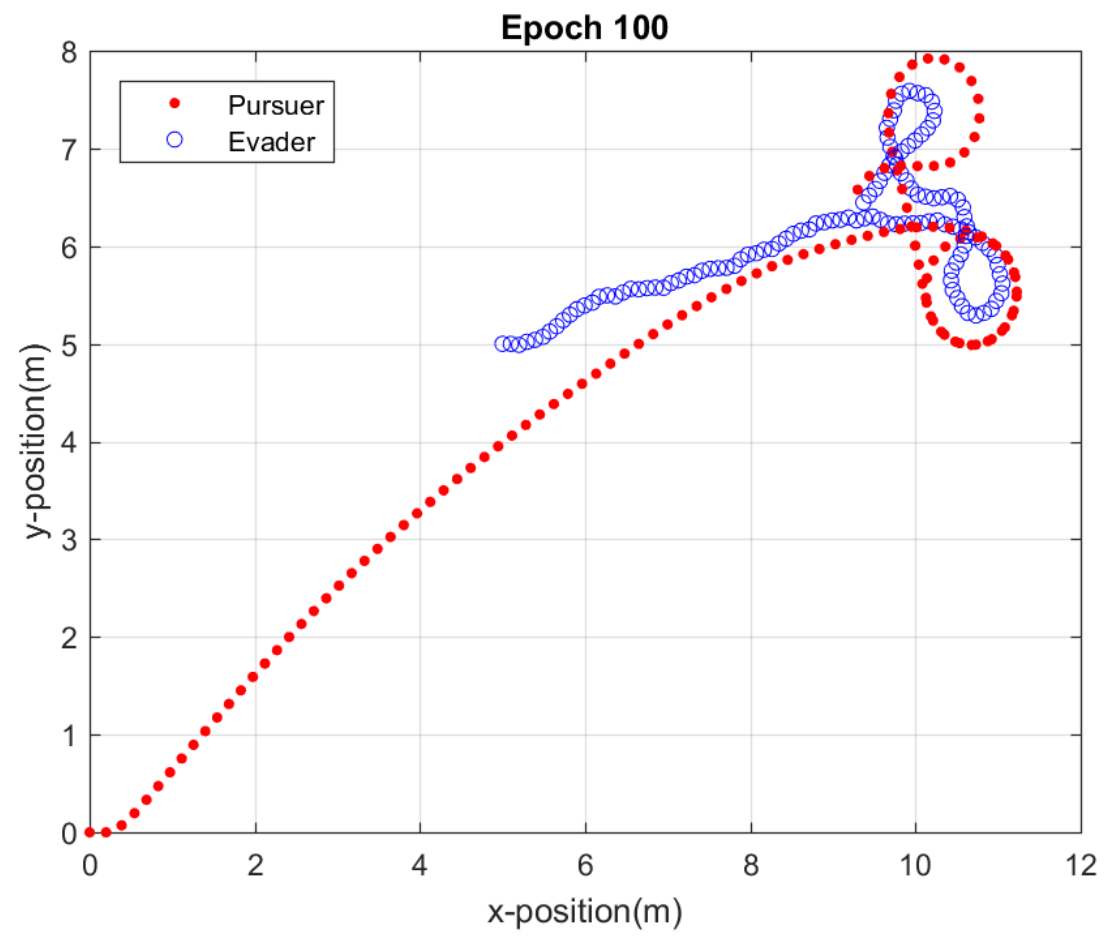

Figure 5.23: Evader delays capture at episode 100 in game of two cars case 3 


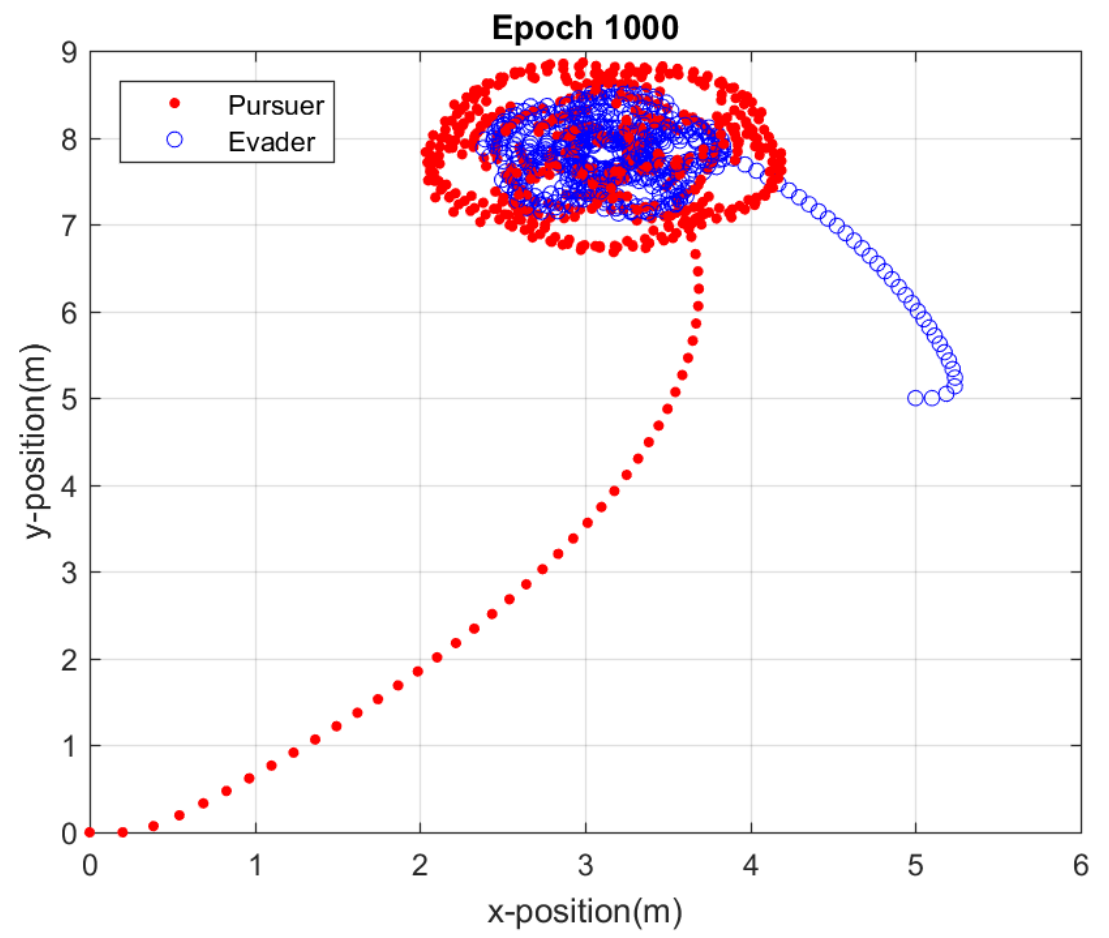

Figure 5.24: Evader escapes at episode 1000 in game of two cars case 3

$D$ is at $V V V C$ are given in Table 5.7.

Table 5.7: The average number of times the rules with premise $V V V C$ (i.e $D=R_{p}$ ) fired during 1000 episodes in game of two cars case 3

\begin{tabular}{|c|c|c|c|}
\hline$\lambda$ & $\mathrm{N}$ & $\mathrm{Z}$ & $\mathrm{P}$ \\
\hline $\mathrm{VVVC}$ & 65344 & 267450 & 215127 \\
\hline
\end{tabular}

The critic parameters of the rules with premise $V V V C$ are shown in Figure 5.25 over 10000 episodes. We observe that all the three parameters converge. From Figure 5.22, the rules: " $\theta_{p e}$ is $N$ and $D$ is $V V V C$ ", " $\theta_{p e}$ is $Z$ and $D$ is $V V V C$ ", " $\theta_{p e}$ is $P$ and $D$ is $V V V C "$ converge to the values $1.4360,1.6100$ and 1.3910 respectively. Table 5.8 gives the summary of the evader's performance in the three scenarios of the 


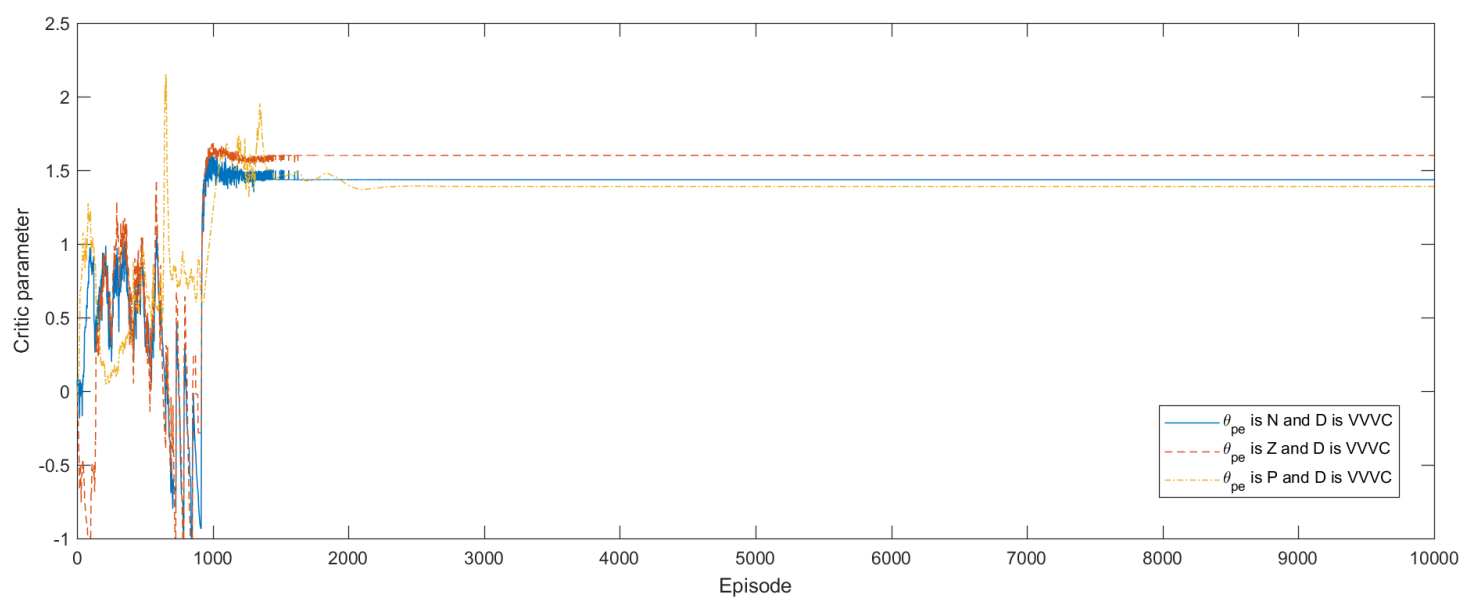

Figure 5.25: Critic's output parameters for rules with premise $V V V C$ in game of two cars case 3



Figure 5.26: Evader's average reward in the game of two cars for case 1, case 2 and case 3 
game of two cars and results of the QLFIS approach used in [1]. We observe that the evader performs better in case 3 when compared to the evaders in cases 1 and 2. However, the evader in case 3 performs better than the evader in the approach used in [1]. The average reward per episode was recorded over 10000 episodes and averaged again over 20 trials for cases 1,2 and 3 shown in Figure 5.26. The evader in case 3 accumulated the most reward from Figure 5.26. The computation times recorded during the last 100 episodes in case 1 , case 2 and case 3 were the same as the computation times recorded in the homicidal chauffeur game case 1 , case 2 and case 3 respectively. Table 5.9 shows the computation times, averaged over 20 trials, for all three cases in comparison with the computation time recorded when using the QLFIS algorithm in [1].

Table 5.8: Summary of the number of episodes taken for the evader to learn its control strategy and percentage number of times the evader escapes in 1000 episodes in the game of two cars

\begin{tabular}{|l|c|l|}
\hline Game of two cars & No. of Episodes to learn & $\%$ No. of Escape \\
\hline Case 1 & No Learning & $0 \%$ \\
\hline Case 2 & 850 & $20 \%$ \\
\hline Case 3 & 700 & $60 \%$ \\
\hline QLFIS in [1] & 900 & $55 \%$ \\
\hline
\end{tabular}

Table 5.9: Summary of the average and standard deviation of the computation times recorded during the last 100 episodes of 20 trials for each case of the homicidal chauffeur game and the game of two cars.

\begin{tabular}{|l|c|l|}
\hline & Average computation time (seconds) & Standard deviation \\
\hline Case 1 & 20.02 & 0.50 \\
\hline Case 2 & 20.80 & 0.62 \\
\hline Case 3 & 20.80 & 0.58 \\
\hline QLFIS in [1] & 40.50 & 0.53 \\
\hline
\end{tabular}




\subsubsection{Homing Missile Guidance}

An inertially guided missile is able to correct its own flight path after it has been launched and without communication with the launch site or any external controller. The missile is fitted with an on-board inertial system that consists of an accelerometer fitted on a gyro-stabilized platform [36]. The purpose of the missile guidance system is to continuously calculate the position of the missile and thereby provide control signals to the missile's flight control system for guidance of the missile to its intended target.

In this simulation, we first run the game using a proportional navigation ratio of $N=2$ and $N=5$ based on Equation 4.21. Then we apply FACL given in Algorithm 6 to the missile. Our objective is to introduce the FACL algorithm into missile guidance and navigation problems. We train the missile to intercept a target moving with a constant velocity. The simulation ends when the missile comes within a fatal distance $(\ell=5 m)$ to the target. The kinematic equations of the missile are described in Equation 4.29. We arbitrarily assume the speed of the missile is $V_{M}=200 \mathrm{~m} / \mathrm{s}$ and the target speed is $V_{T}=100 \mathrm{~m} / \mathrm{s}$. The mass of the missile is also arbitrarily selected as $m=100 \mathrm{~kg}$. We also assume the target moves at a constant velocity and does not take evasive action.

We want the rate of change of the missile's flight path $\dot{\theta}_{M}$ to follow the rate of change of the LOS vector. We define a reward function $R_{t+1}$ given in Equation 5.8 as

$$
R_{t+1}=2 e^{-\left(\epsilon^{2}\right)}-1
$$

where $\epsilon=\dot{\theta}_{M}-\dot{\Omega}$. The reward $R_{t+1}=1$ when the difference $\epsilon$ is zero and $R_{t+1}=-1$ when $\epsilon$ is large. 
The initial positions and direction of the missile and target respectively are $\left(x_{m}=0, y_{m}=0, \theta_{m}=0 \mathrm{rad}\right)$ and $\left(x_{t}=500 \mathrm{~m}, y_{t}=500 \mathrm{~m}, \theta_{t}=0 \mathrm{rad}\right)$. We select only one input to the missile's fuzzy inference system which is the Cartesian distance $D \in[0,100]$ between the missile and the target. We use the the triangular membership functions in Figure 5.8 but over the universe of discourse $D \in[0,100]$. The membership degree $\mu_{D}$ of any value beyond the lower and upper limits of $D$ saturate at $\mu_{D}=1$. The control output of the actor is the normal force $F_{N}(t)$ required for the missile to successfully intercept the target. We run the simulation 20 times for 500 learning episodes with 100 time steps per episode. The simulation result which is

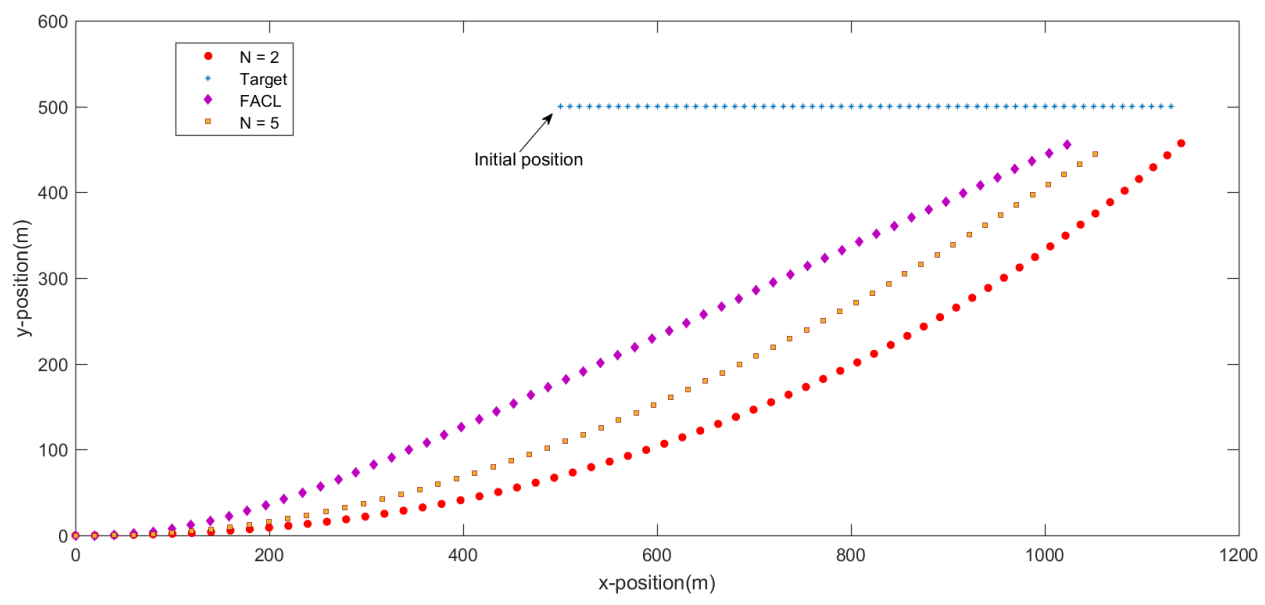

Figure 5.27: Missile trajectory for PN Law (at $N=2$ and $N=5$ ) and FACL(after 500 learning episodes)

Table 5.10: Real flight time (in seconds) taken for missile to intercept target with proportional navigation (at $N=2$ and $N=5$ ) and with FACL

\begin{tabular}{|l|c|l|}
\hline Time $(N=2)$ & Time $(N=5)$ & Time (FACL) \\
\hline $5.70 \mathrm{~s}$ & $5.50 \mathrm{~s}$ & $5.20 \mathrm{~s}$ \\
\hline
\end{tabular}

presented in Figure 5.27 shows the trajectory of the missile when using PN Law at 
$N=2, N=5$ and when the FACL was applied to the missile. The real flight time taken for the missile to intercept the target is given in Table 5.10. The missile learns to intercept the target at about episode 300 with an average real flight capture time of 5.20 seconds and a standard deviation of 0.38 over 20 trials. The missile was able to intercept the target in lesser time with FACL.

\subsubsection{Discussion}

In this chapter, we presented the applications of $\mathrm{Q}(\lambda)$-learning (without eligibility traces) and fuzzy actor-critic learning (FACL) in the homicidal chauffeur game and the game of two cars. We focused on the evader learning its control strategy although, both pursuer and evader were learning simultaneously. We used only triangular membership functions in the fuzzy inference system and updated only the fuzzy output parameters. We observed whether increasing the number of membership functions will speed up the evader's learning process and the effects of this increase on the computation time. We compared our results to the results obtained when using the method in [4] and [1]. Increasing the membership functions over only the evader's input distance, which is very significant to the evader, the evader was able to learn its control strategy. The evader using our approach performed better in terms of learning speed and computation time than when the $\mathrm{Q}(\lambda)$-learning in $[1,4]$ was used.

We also studied whether the fuzzy actor-critic algorithm would perform better

against the proportional navigation method used in homing missile-target interceptions. The FACL algorithm performed slightly better than the proportional navigation method. 


\section{Chapter 6}

\section{Conclusion}

Reinforcement learning has been used to successfully train pursuers in pursuit-evasion games. However, there is insufficient studies on how to successfully train the evader in a pursuit evasion game using reinforcement learning.

In Chapter 2, we examined fuzzy logic systems and how they can be used as function approximators. We especially look at triangular membership functions because they are simple to use and can minimize the amount of computation required, if structured a specific way.

We discussed reinforcement learning (RL) in Chapter 3 and how reinforcement learning problems are formalized using Markov decision processes. In addition, we discussed some reinforcement learning methods such as; Q-learning and actor-critic learning. Reinforcement learning methods are originally designed for small discrete state and action spaces. Algorithms like the $\mathrm{Q}(\lambda)$-learning fuzzy inference system (QLFIS) and fuzzy actor-critic learning (FACL) combine RL methods with fuzzy

inference systems so that RL can be applied to problems that have continuous or large discrete state and action spaces.

In Chapter 4, we discussed the homicidal chauffeur game and the game of two cars. We presented the time optimal strategies for both pursuer and evader in both games. 
The control output for the pursuer and the evader in both games is their respective steering angles. We introduced an inertial homing missile guidance problem and the proportional navigation guidance law. The control output in the inertial homing missile guidance problem is a normal force.

In Chapter 5, we investigate how the evader in the homicidal chauffeur game and the game of two cars can learn its optimal strategy. The FACL algorithm was used to train both pursuer and evader to learn the game simultaneously. However, we focus more on the evader learning its control strategy. We investigated how the evader can learn its control strategy using inputs that have not been considered in literature for both games such as; the line-of-sight (LOS) of the pursuer to the evader, and the angle difference between the orientation of the pursuer and the evader. We also observed the performance of the evader when using triangular membership functions and when updating only the output parameters. The evaders performance improved significantly by simply increasing the number of membership functions. The simulation results in Chapter 5 show that the evader successfully learned to escape in both games. The evader's was able to learn its control strategy in fewer episodes when compared with the QLFIS algorithm and method used in [1]. We observed that our approach takes less time to compute that the method in [1]. The FACL algorithm was applied to the inertial homing missile and demonstrated a better trajectory than when using the proportional navigation method. In this thesis, we only considered a point mass inertial missile model. In future research, FACL can be applied to a missile with more complex dynamics. 


\section{List of References}

[1] B. Al Faiya, Learning in pursuit-evasion differential games using reinforcement fuzzy learning. PhD thesis, Carleton University, 2012.

[2] R. Isaacs, Differential games: a mathematical theory with applications to warfare and pursuit, control and optimization. Courier Corporation, 1999.

[3] R. S. Sutton and A. G. Barto, Reinforcement learning: An introduction. MIT press, 2018.

[4] F. D. Sameh and M. S. Howard, "Q $(\lambda)$-learning fuzzy logic controller for differential games," in 2010 10th International Conference on Intelligent Systems Design and Applications, pp. 109-114, IEEE, 2010.

[5] Y. Duan et al., "Fuzzy reinforcement learning and its application in robot navigation," in 2005 International Conference on Machine Learning and Cybernetics, vol. 2, pp. 899-904, IEEE, 2005.

[6] L. Jouffe, "Fuzzy inference system learning by reinforcement methods," IEEE Transactions on Systems, Man, and Cybernetics, Part C (Applications and Reviews), vol. 28, no. 3, pp. 338-355, 1998.

[7] Z. Chi, H. Yan, and T. Pham, Fuzzy algorithms: with applications to image processing and pattern recognition, vol. 10. World Scientific, 1996.

[8] K. Hirota, "Application of fuzzy theory to home appliances," in Industrial Applications of Fuzzy Technology, pp. 283-310, Springer, 1993.

[9] X. Dai, C.-K. Li, and A. B. Rad, "An approach to tune fuzzy controllers based on reinforcement learning for autonomous vehicle control," IEEE Transactions on Intelligent Transportation Systems, vol. 6, no. 3, pp. 285-293, 2005. 
[10] L. P. Kaelbling, M. L. Littman, and A. W. Moore, "Reinforcement learning: A survey," Journal of artificial intelligence research, vol. 4, pp. 237-285, 1996.

[11] M. van Otterlo and M. Wiering, "Reinforcement learning and markov decision processes," in Reinforcement Learning, pp. 3-42, Springer, 2012.

[12] W. M. Van Buijtenen, G. Schram, R. Babuska, and H. B. Verbruggen, "Adaptive fuzzy control of satellite attitude by reinforcement learning," IEEE Transactions on Fuzzy Systems, vol. 6, no. 2, pp. 185-194, 1998.

[13] C. J. Watkins and P. Dayan, "Q-learning," Machine learning, vol. 8, no. 3-4, pp. 279-292, 1992.

[14] M. Boumehraz and K. Benmahammed, "Tuning fuzzy inference systems by qlearning," 2014.

[15] H. Chen, J. Chen, W. Zhang, and H. Liu, "Analysis of a new pursuit-evasion game based on game theory," in 2015 11th International Conference on Natural Computation (ICNC), pp. 875-880, IEEE, 2015.

[16] S. M. LaValle, Planning algorithms. Cambridge university press, 2006.

[17] S. H. Lim, T. Furukawa, G. Dissanayake, and H. F. D. Whyte, "A time-optimal control strategy for pursuit-evasion games problems," in Proceeding. of the 2004 IEEE International Conference on Robotics and Automation, (New Orleans, LA), Apr. 2004.

[18] A. MERZ, "The homicidal chauffeur," AIAA Journal, vol. 12, pp. 259-260, March 1974.

[19] S. F. Desouky and H. M. Schwartz, "Learning in n-pursuer n-evader differential games," in 2010 IEEE International Conference on Systems, Man and Cybernetics, pp. 4069-4074, IEEE, 2010.

[20] J. Li, L. Yang, Y. Qu, and G. Sexton, "An extended takagi-sugeno-kang inference system (tsk+) with fuzzy interpolation and its rule base generation," Soft Computing, vol. 22, no. 10, pp. 3155-3170, 2018.

[21] K. M. Passino, S. Yurkovich, and M. Reinfrank, Fuzzy control, vol. 42. Citeseer, 1998.

[22] L.-X. Wang, A course in fuzzy systems. Prentice-Hall press, USA, 1999. 
[23] J.-S. R. Jang, C.-T. Sun, and E. Mizutani, Neuro-fuzzy and soft computing; a computational approach to learning and machine intelligence. CUMINCAD, 1997.

[24] L. A. Zadeh, "On fuzzy algorithms," in Fuzzy Sets, Fuzzy Logic, And Fuzzy Systems: Selected Papers by Lotfi A Zadeh, pp. 127-147, World Scientific, 1996.

[25] L. A. Zadeh, "Information and control," Fuzzy sets, vol. 8, no. 3, pp. 338-353, 1965.

[26] E. H. Mamdani and S. Assilian, "An experiment in linguistic synthesis with a fuzzy logic controller," International journal of man-machine studies, vol. 7, no. 1, pp. 1-13, 1975.

[27] T. Takagi and M. Sugeno, "Fuzzy identification of systems and its applications to modeling and control," IEEE transactions on systems, man, and cybernetics, no. 1, pp. 116-132, 1985.

[28] H. M. Schwartz, Multi-agent machine learning: A reinforcement approach. John Wiley \& Sons, 2014.

[29] M. L. Littman, "Value-function reinforcement learning in markov games," Cognitive Systems Research, vol. 2, no. 1, pp. 55-66, 2001.

[30] V. R. Konda and J. N. Tsitsiklis, "Actor-critic algorithms," in Advances in neural information processing systems, pp. 1008-1014, 2000.

[31] T. Basar and G. J. Olsder, Dynamic Noncooperative Game Theory. Academic Press, New York: SIAM, 1999.

[32] D. Solomon Raj, "Performance evaluation of proportional navigation guidance for low-maneuvering targets," International Journal of Scientific \& Engineering Research, vol. 5, no. 9, pp. 93-99, 2014.

[33] D. Cho, H. J. Kim, and M.-J. Tahk, "Fast adaptive guidance against highly maneuvering targets," IEEE Transactions on Aerospace and Electronic Systems, vol. 52, no. 2, pp. 671-680, 2016.

[34] U. O. City, "Gunner's Mate Missile." https://www.okieboat.com/GMM/GMM\% 203\%20and $\% 202 \% 20$ CHAPTER $\% 204 \% 20$ Missile $\% 20$ Guidance $\% 20$ and $\% 20$ Control. pdf, 1972. [Online; accessed 25-July-2019]. 
[35] P.-J. Yuan and S.-C. Hsu, "Solutions of generalized proportional navigation with maneuvering and nonmaneuvering targets," IEEE Transactions on Aerospace and Electronic Systems, vol. 31, no. 1, pp. 469-474, 1995.

[36] T. Karthikeyan and A. Kapoor, Guided Missiles. Defence Scientific Information \& Documentation Centre, Defence Research ..., 1991. 